Trans. zool. Soc. Lond. (1978) 34, 263-345

\title{
The phylogeny of the Charadriiformes (Aves): a new estimate using the method of character compatibility analysis
}

\author{
Joseph G. Strauch, JR. \\ Museum of Zoology, The University of Michigan, \\ Ann Arbor, Michigan 48109, U.S.A.*
}

(Accepted 12 October 1977)

(With 36 figures in the text)

Published for

THE ZOOLOGICAL SOCIETY OF LONDON

by

ACADEMIC PRESS

* Present address: Science Applications Inc, 2760 29th St., Suite 209, Boulder, Colorado 80302, U.S.A. 


\section{The phylogeny of the Charadriiformes (Aves): a new estimate using the method of character compatibility analysis}

Character state trees were devised for 70 mainly skeletal characters of 227 species of charadriiform birds. Character compatibility analysis (described herein) was used to determine the largest sets of mutually compatible characters in the data set. Largest sets of mutually compatible characters were chosen as the best estimators of the phylogenetic history of the order. Smaller and smaller monophyletic groups (as identified by previous analysis of the next larger monophyletic group) were analysed to find locally largest sets of mutually compatible characters until the cladistic information in the data set was exhausted.

The results of these analyses indicate that the Charadriiformes consist of three phyletic lines (here treated as suborders): the Scolopaci, the Charadrii and the Alcae. The Scolopaci consist of the birds usually included in the families Jacanidae, Rostratulidae, Scolopacidae, Phalaropodidae and Thinocoridae. The Charadrii consist of two major phyletic branches: one leading to the Lari and the other to the line discussed below. The second branch of the Charadrii gives rise to five lineages: one which leads to Dromas, a second to Pluvianellus and Chionis, a third to Pluvianus and the Burhinidae, a fourth to the Glareolidae, and a fifth to the plovers, lapwings, oystercatchers, Ibisbill, avocets, and stilts.

Evidence which supports these findings, that which contradicts them, and relationships in need of further study are discussed. 


\section{CONTENTS}

Page

Introduction

The method of character compatibility. $\quad \begin{array}{llllllllllllll} & \ldots & \ldots & \ldots & \ldots & \ldots & \ldots & & \ldots & 271\end{array}$

$\begin{array}{llllllllllllllll}\text { Characters . } & \ldots & \ldots & \ldots & \ldots & \ldots & \ldots & \ldots & \ldots & \ldots & \ldots & \ldots & 279\end{array}$

$\begin{array}{llllllllllllll}\text { Skull characters } & \ldots & \ldots & \ldots & \ldots & \ldots & \ldots & \ldots & \ldots & \ldots & \ldots & 289\end{array}$

$\begin{array}{llllllllllllll}\text { Mandible characters. } & \ldots & \ldots & \ldots & \ldots & \ldots & \ldots & \ldots & \ldots & \ldots & 301\end{array}$

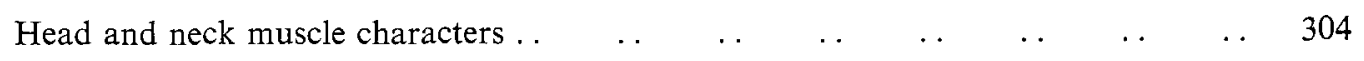

$\begin{array}{lllllllllllll}\text { Axial skeleton characters } & \ldots & \ldots & \ldots & \ldots & \ldots & \ldots & \ldots & \ldots & \ldots & 304\end{array}$

$\begin{array}{llllllllllllll}\text { Pectoral girdle characters } & \ldots & \ldots & \ldots & \ldots & \ldots & \ldots & \ldots & \ldots & \ldots & 306\end{array}$

$\begin{array}{llllllllllllll}\text { Wing characters } & \ldots & \ldots & \ldots & \ldots & \ldots & \ldots & \ldots & \ldots & \ldots & \ldots & 312\end{array}$

$\begin{array}{lllllllllllll}\text { Synsacrum characters } & \ldots & \ldots & \ldots & \ldots & \ldots & \ldots & \ldots & \ldots & \ldots & 314\end{array}$

$\begin{array}{llllllllllllll}\text { Hind } \operatorname{limb} \text { characters } & \ldots & \ldots & \ldots & \ldots & \ldots & \ldots & \ldots & \ldots & \ldots & 318\end{array}$

$\begin{array}{llllllllllllllll}\text { Results } & \ldots & \ldots & \ldots & \ldots & \ldots & \ldots & \ldots & \ldots & \ldots & \ldots & \ldots & \ldots & 322\end{array}$

$\begin{array}{lllllllllllll}\text { The Charadriformes } & \ldots & \ldots & \ldots & \ldots & \ldots & \ldots & \ldots & \ldots & \ldots & 322\end{array}$

$\begin{array}{lllllllllll}\text { The suborders of the Charadrifformes } & \ldots & \ldots & \ldots & \ldots & \ldots & \ldots & \ldots & 323\end{array}$

$\begin{array}{lllllllllllll}\text { The Scolopaci . } & \ldots & \ldots & \ldots & \ldots & \ldots & \ldots & \ldots & \ldots & \ldots & 323\end{array}$

The Rostratulidae, Scolopacidae, Phalaropodidae, and Thinocoridae . 325

The Rostratulidae, Scolopacidae, and Phalaropodidae $\quad \ldots \quad$. . $\quad$. 326

$\begin{array}{llllllllllll}\text { The Charadrii } & \ldots & \ldots & \ldots & \ldots & \ldots & \ldots & \ldots & \ldots & \ldots & \ldots & 327\end{array}$

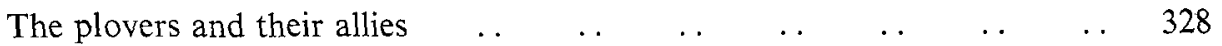

Analysis of the Charadrii using hypothetical ancestors . $\quad \begin{array}{lllllllllll} & \ldots & \ldots & \ldots & \ldots & 328\end{array}$

Summary of the cladistic relationships among the Charadriformes $\ldots \ldots \ldots$. . $\quad 330$

$\begin{array}{llllllllllllllll}\text { Discussion . } & \ldots & \ldots & \ldots & \ldots & \ldots & \ldots & \ldots & \ldots & \ldots & \ldots & \ldots & 333\end{array}$

$\begin{array}{lllllllllllllll}\text { Acknowledgements } & \ldots & \ldots & \ldots & \ldots & \ldots & \ldots & \ldots & \ldots & \ldots & \ldots & 341\end{array}$

$\begin{array}{llllllllllllllll}\text { References . . } & \ldots & \ldots & \ldots & \ldots & \ldots & \ldots & \ldots & \ldots & \ldots & \ldots & \ldots & 342\end{array}$ 


\section{INTRODUCTION}

Much of the literature of systematics deals with the identification of characters which are good estimators of phylogenetic history. Early systematists had little more than their own insights to help them choose the characters which best indicate relationships. The stability of much of zoological classification is testimony to their good judgment in their choices. Their methods, however, have made it difficult or impossible for others to follow or repeat the steps from observations of specimens to the statements of relationship among taxa. The subjectivity of the intuitive method has led many systematists to reject classical phylogenetic studies and to accept phenetic methods (Sneath \& Sokal, 1972). Pheneticists argue that classifications should be based on overall resemblance without regard to phylogeny. Those interested in estimating phylogenetic history, on the other hand, have long held that some characters are better than others for reconstructing phylogenies and that the major problem is to identify them. In recent years there has been a theoretical and methodological revolution in the estimation of phylogenetic relationships (Camin \& Sokal, 1965; Hennig, 1966; Fitch \& Margoliash, 1967; Kluge \& Farris, 1969; Estabrook, 1972). Few of these new ideas, however, have been used in avian systematics (Selander, 1971; Cracraft, 1972).

In this study I have used the method of character compatibility (Estabrook, 1972; McMorris, 1975; Estabrook, Strauch \& Fiala, 1977) to estimate the branching patterns (cladistic relationships) of the phylogenetic history of the Charadriiformes. This method is based on current evolutionary theory, and its methods of analysis have been shown to be mathematically exact (Estabrook, Johnson \& McMorris, 1975, 1976a, b). Although the terminology and methodology of this method might at first seem foreign to many systematists, in truth it merely formulates in mathematical terms traditional systematic practices such as using the largest set of characters which agree to define taxonomic goups and treating some characters as more important indicators of relationships in some groups than in others. Unlike the Wagner Tree parsimony methods (Kluge \& Farris, 1969) and the methods used by the followers of Hennig (e.g. Cracraft, 1974), character compatibility analysis allows precise and objective identification of the best characters used in a study. The relationship between the method of character compatibility and traditional methods is discussed in more detail by Estabrook, Strauch \& Fiala (1977).

The avian order Charadriiformes includes birds commonly known as waders, gulls, terns and auks, as well as several less familiar types. This order is especially suitable for a phylogenetic study because it has given rise to many diverse types, members of which are found in almost all terrestrial and aquatic habitats, and because there have been several recent extensive studies of their systematics.

All the birds currently included in the Charadriiformes were first grouped together by Huxley (1867) on the basis of skull characteristics. The major debates among bird systematists concerning charadriiform birds since Huxley's work have centred around several problems: possible relationships among the Laridae, Procellariiformes, Gaviidae, 
and Alcidae (Sclater, 1880; Shufeldt, 1891), possible relationships among the Gruiformes and Charadriiformes, especially between the Otididae and Burhinidae (Garrod, 1873; Sclater, 1880; Sharpe, 1891; Lowe, 1931a; Boetticher, 1934; Stresemann, 1959) and between the Rallidae and the Jacanidae (Forbes, 1881; Lowe, 1925; Stresemann, 1959); the relationships of the Thinocoridae (Seebohm, 1895; Shufeldt, 1891; Mathews \& Iredale, 1921; Lowe, 1922, 1923; Hanke \& Niethammer, 1955; Sibley, Corbin \& Ahlquist, 1968), and the question of whether the Pteroclidae are members of the Charadriiformes (Huxley, 1868; Garrod, 1874; Sclater, 1880; Gadow, 1893; Beddard, 1898; Maclean, 1967, 1969; Stegmann, 1968, 1969; Olson, 1970).

Between 1914 and 1933 Percy R. Lowe published a series of papers on the anatomy and relationships of the Charadriiformes, especially waders. Lowe's contributions include descriptions of the anatomy of several previously unstudied forms and discussions of the states of several characters in many different species. His decisions regarding relationships, however, are suspect because he relied heavily on only a few characters, such as colour pattern, supraorbital grooves, and the morphology of the quadrato-tympanic articulation.

Kozlova (1961) and her collaborator Yudin (1965) proposed a phylogeny of the Charadriiformes based on their "ecologico-morphology" method. Using information on behaviour, ecology, and the morphology of the head and wing, they began with the assumption that the genus Pluvialis represents the archetype of the Charadriiformes from which more specialized charadriiform groups radiated. While they provide much valuable information on charadriiform morphology, the vagueness of their methods for inferring relationships and their use of much fragmentary and anecdotal evidence make many of their conclusions of doubtful value.

In a survey of 24 orders of birds Kitto \& Wilson (1966) found that charadriiform birds have a unique S-malate dehydrogenase, the mobility of which is $55 \%$ that of most other birds. Their results indicate that the Jacanidae and Burhinidae are members of the Charadriiformes while the Pteroclidae are not.

Jehl (1968), like Lowe (1915a), surveyed the plumage patterns of the downy young of waders. His conclusions, based on subjective estimates of similarity of overall patterns and the relationships implied by them, are in need of an objective reevaluation.

Burton (1974) in an extensive survey of the feeding apparatus of waders discussed some of the systematic implications of his findings. Ahlquist (1974) used the IFPA (isoelectric focusing in polyacrylamide) patterns of egg-white proteins and two-dimensional electrophoresis maps of ovalbumin peptide digests to estimate relationships among the Charadriiformes. Unfortunately, his conclusions can be accepted only as suggestive, since he made dubious assumptions concerning the homologies among the spots found on the peptide maps for different species and used analytical methods inappropriate to his data.

Before an estimate of phylogenetic relationships is made, a group of organisms is chosen which is believed to represent the living descendants of a single ancestor. Thus even before the phylogenetic history can be estimated, some estimate of phylogenetic history is already assumed at a higher taxonomic level. I agree with Colless $(1967,1969 a, b)$ that one is forced to start with some sort of phenetic estimate of relationship as a beginning of a phylogenetic study.

Rather than arbitrarily follow one of the current classifications of the Charadriiformes, which disagree on which families are members of the order, I chose to make an independent determination of its membership by means of a Prim Network (Prim, 1957; Sneath \& 
Sokal, 1972) analysis of 46 mostly skeletal characters of 64 species chosen to represent much of the diversity in the Gruiformes, Charadriiformes, Columbiformes, Gaviiformes, and Podicipediformes (Strauch, 1976). The results of that analysis support the hypothesis that the Charadriiformes are a monophyletic group consisting of the following families: Jacanidae, Rostratulidae, Haematopodidae, Charadriidae, Scolopacidae, Recurvirostridae, Phalaropodidae, Dromadidae, Burhinidae, Glareolidae, Thinocoridae, Chionididae, Stercorariidae, Laridae, Rynchopidae, and Alcidae. The analysis gave no evidence that the Jacanidae are similar to the Rallidae, that the Thinocoridae are close to any of the Gruiformes, or that the Burhinidae are close to the Otididae.

The nomenclature used in the text is basically that of Peters (1934) with the exception that the generic and specific names used by Jehl (1968) are followed for most of the waders. Character names follow Bock \& McEvey (1969), supplemented by Bams (1956), Burton (1971, 1974), Cracraft (1968), George \& Berger (1966), Holmann (1961), Howard (1929), Lebedinsky (1913), Rand (1954) and Zusi \& Jehl (1970).

\section{THE METHOD OF CHARACTER COMPATIBILITY}

Character compatibility is a method for identifying cladistically useful characters. Since this approach is unfamiliar to most investigators, a brief and simplified summary of the underlying theory is given below. (See Estabrook, 1972; Estabrook, Johnson \& McMorris, 1975, 1976a, $b$; McMorris, 1975; Estabrook, Strauch \& Fiala, 1977.)

When a worker assembles specimens of organisms whose evolutionary history he wishes to estimate, he makes certain assumptions about the degrees of relatedness among them and about their phylogenetic history. The assumptions made here are that the organisms being studied are members of a set of evolutionary units (EUs) whose cladistic history can be represented by a tree.

There is disagreement about what the set of evolutionary units should be. Hennig $(1965,1966)$ argued that a set of evolutionary units consists of groups of organisms alive at the same time. The concept of a set of evolutionary units used here, however, is broadened to include the set's own most recent common ancestor and all the units arising from that common ancestor, contemporary or ancestral. It is therefore necessary to make estimates of the unrepresented units, just as hypothetical intermediates must be estimated in the Wagner Tree parsimony methods (Kluge \& Farris, 1969). A hypothetical representation of the evolutionary history of a set of evolutionary units is illustrated in Fig. 1A. Each phyletic line segment of the tree represents a discrete (monophyletic) evolutionary unit; it represents genetic continuity through time. To satisfy the requirements that each unit be discrete and that no two units have any organism in common, a few generations close to a branching point of a phyletic line, or joining distinct units consecutive along the same phyletic line, are excluded from evolutionary units.

Once the units have been defined, it is necessary to define the relationship among them that we wish to study. The relationship A, "is an ancestor of", defined for S', the set of evolutionary units described above, is of special phylogenetic significance. This relationship is mathematically defined so that for any units in $S^{\prime}$ the following are always true:

1. $a \mathrm{~A} a$ (read, " $a$ is an ancestor of $a$ "), i.e. $a$ is its own ancestor;

2. if $a \mathrm{~A} b$ and $b \mathrm{~A} a$ are both true, then $a$ is the same unit as $b$;

3. if $a \mathrm{~A} b$ and $b \mathrm{~A} c$ are both true, then $a \mathrm{~A} c$ is also true. 


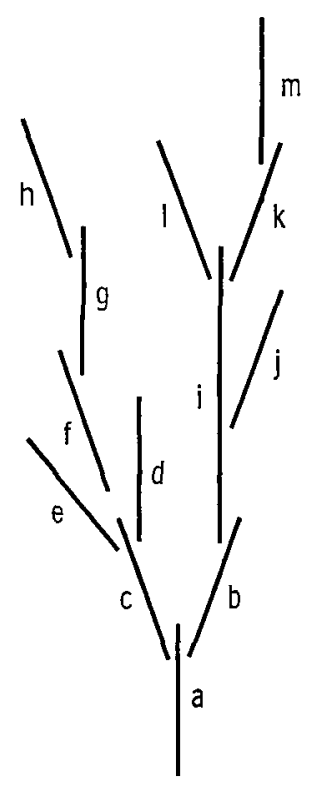

A

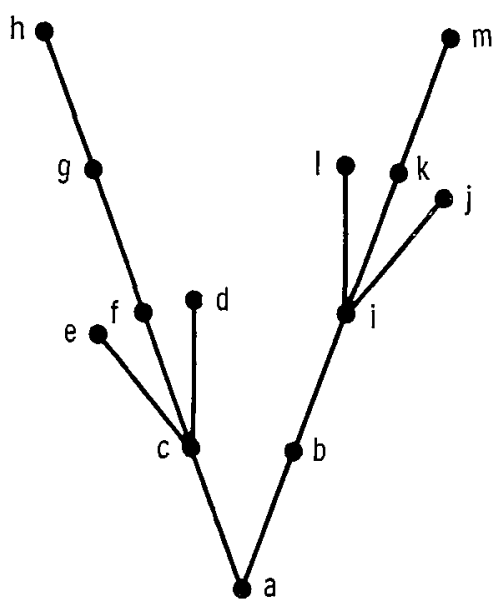

B

FIG. 1. Representations of evolutionary history. A. a tree representing the evolutionary history of a hypothetical set of evolutionary units; B. a Hasse diagram for the relationship is an ancestor of among the set of evolutionary units in (A).

The relationship A corresponds to an algebraic tree partial order (Estabrook, 1968, 1972).

The relationship A can be illustrated with a Hasse diagram. (A Hasse diagram in abstract algebra is a diagram which represents a partially ordered set (Moore, 1967).) Hasse diagrams can be used to represent phylogenetic trees and are graphic representations of the relationship for the set of units placed on them. The nodes on a Hasse diagram represent phyletic lines; these nodes are connected by lines (called edges), which signify that the nodes are related in a specific manner. Figure $1 \mathrm{~B}$ is the Hasse diagram of the tree partial order A for the set of EUs $S^{\prime}$ shown in Fig. 1A. The partially ordered elements of $\mathrm{S}^{\prime}$ are connected by lines such that whenever $a \mathrm{~A} b$, a path leads from $a$, possibly through other elements which lie between $a$ and $b$ in the diagram, to $b$, in a direction which is always toward the top of the diagram. The line between $a$ and $c$ is read, " $a$ is an ancestor of $c$ ".

FIG. 2. Phylogenetic implications of Hasse diagrams. A. a Hasse diagram on which real EU $b$ is shown to be the ancestor of real EU $a$. Such a diagram is best interpreted as indicating that EUs $a$ and $b$ share a common ancestor, but none of the characters used in the study distinguishes $b$ from the most recent common ancestor it shares with $a$ (middle figure). The two figures on the right are more complex interpretations of the possible relationship between $a$ and $b$ which would be of interest only if a third EU had to be included on the diagram. B. The figures on the right represent possible phylogenetic histories which are consistent with the Hasse diagram on the left. Solid circles represent real EUs, open circles represent hypothetical ancestral EUs, solid internodes represent character state transitions defined by characters included in a study, and dashed internodes represent possible transitions but are not defined by any character in the study. 

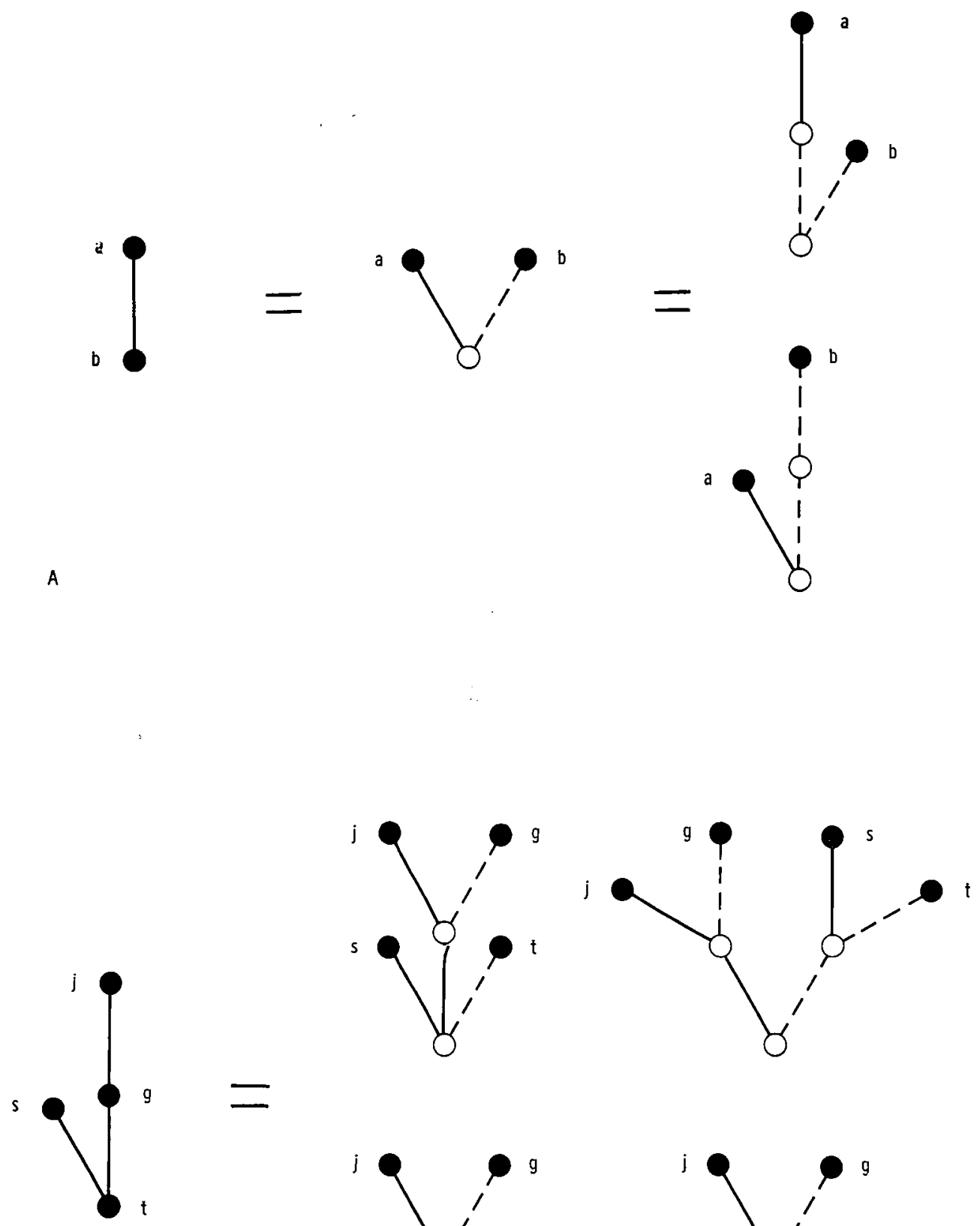

B
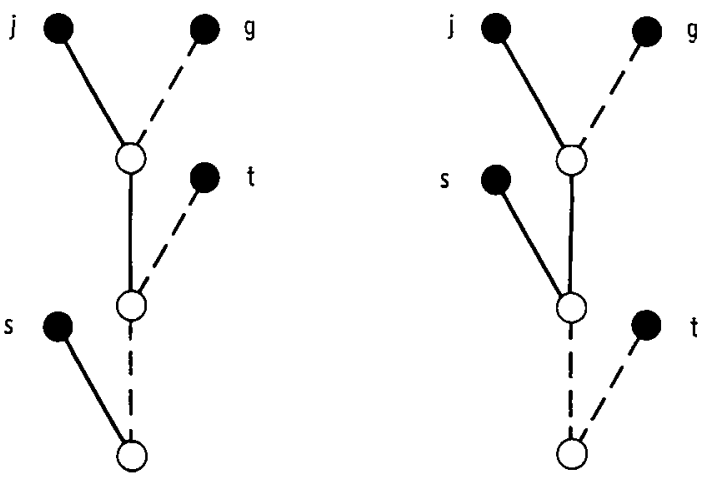

FIG. 2. 
All the trees presented in this study are in the form of Hasse diagrams. The phylogenetic implications of these diagrams are illustrated in Fig. 2. On these diagrams solid circles represent real EUs ( $a$ and $b$ in Fig. 2A), and open circles represent hypothetical EUs generated by the analyses. When a real EU is connected directly to another real EU on the tree, such as $a$ connected to $b$ in Fig. 2, it does not imply that one EU is the ancestor of the other but only that the characters defining the tree do not distinguish some EUs from their ancestors. Thus in Fig. 2, $a$ and $b$ share a common ancestor, but none of the characters used to devise the tree distinguishes $b$ from the ancestor it shares with $a$. The middle figure in Fig. 2A shows this relationship with a dashed line for the internode between $b$ and the ancestor it shares with $a$; the figures on the right in Fig. 2A show other possible phylogenetic interpretations of the figure on the left. A more complex tree and four of the possible phylogenies it could represent are shown in Fig. 2B.

Central to estimating the evolutionary history of a set of EUs is finding a basis for comparison and then structuring a comparative scheme which translates statements of similarities and differences into statements about the relative recency of common ancestry of the EUs. A basis for such a comparison is called a "character". A character can be thought of algebraically as a function defined for the study collection $S$, or, ideally for the set of EUs, S', for which the values are descriptions. The members of the set descriptions are called "character states". The character "toe webbing", for example, might have character states "toes not webbed" and "toes webbed". Thus if the character K is a character for $\mathrm{S}$, and $a$ is contained in $\mathrm{S}, \mathrm{K}(a)$ is the description of $a$ based on or made by character K. For the EU "American avocet (Recurvirostra americana)" contained in the set "Charadriiformes", the character "toe webbing" would have the description "toes webbed". A character may be construed as an equivalence relation on S (or S'). An equivalence relation asserts that two things are equivalent with respect to some consideration. Thus the four living species of avocets are equivalent with respect to toe webbing.

If $a, b$, and $c$ are EUs and $\mathrm{E}$ is an equivalence relation on $\mathrm{S}$ or $\mathrm{S}^{\prime}$, then $\mathrm{E}$ is defined as having the following properties:

1. $a \mathrm{E} a$ is always true;

2. if $a \mathrm{E} b$ is true, then $b \mathrm{E} a$ must be true;

3. if $a \mathrm{E} b$ and $b \mathrm{E} c$ are true, then $a \mathrm{E} c$ must also be true.

By placing $a$ and $b$ in the same group whenever $a \mathrm{E} b$, E determines a nonhierarchical grouping of the members of $S$ or $S^{\prime}$. These groups are the equivalence classes of $\mathrm{E}$. Character $\mathrm{K}$ determines an equivalence relation $a \mathrm{E} b$ if and only if $\mathrm{K}(a)=\mathrm{K}(b)$. The equivalence classes of $\mathrm{K}$ are called character states.

Characters useful for estimating the true evolutionary history should be divergent, that is, all the EUs belonging to a given state $\mathrm{K}(a)$ must have evolved from a most recent common ancestor with $\mathrm{K}(a)$ with no change in the property characteristic of $\mathrm{K}(a)$ (no reversals), and changes in the states of $\mathrm{K}$ must have occurred only on phyletic lines leading directly to the recent common ancestors of the states of $\mathrm{K}$ themselves (unique origin). Thus for the character "toe webbing" to be divergent, all avocets with webbed toes must have evolved from a most recent common ancestor with webbed toes, all the organisms along the phyletic lines leading from the common ancestor of avocets to the recent species of avocets must also have had webbed toes, and finally, the change in the character "toe webbing" which gave rise to webbed toes occurred only on the phyletic line leading directly to the most recent common ancestor of all avocets. 
To use characters to estimate evolutionary history, the properties of characters which are ideally related to true history must be specified. A cladistic character is a character which includes estimates of evolutionary trends and is therefore itself an estimate of evolutionary history. A relationship $\mathrm{P}$, is more primitive than, is defined for the set of character states of $\mathrm{K} . \mathrm{P}$ is a tree partial order which estimates the evolutionary trends among the states of $\mathrm{K}$. The Hasse diagram of $\mathrm{P}$ is a character state tree (Fig. 3). If $\mathrm{K}$ is to be used to estimate evolutionary history, then there must be a relationship between the relationship A, is an ancestor of, and the relationship $\mathrm{P}$, is more primitive than. For $\mathrm{A}$ to be ideally related to $\mathrm{P}$, each equivalence class of $\mathrm{K}$ should have the following properties:

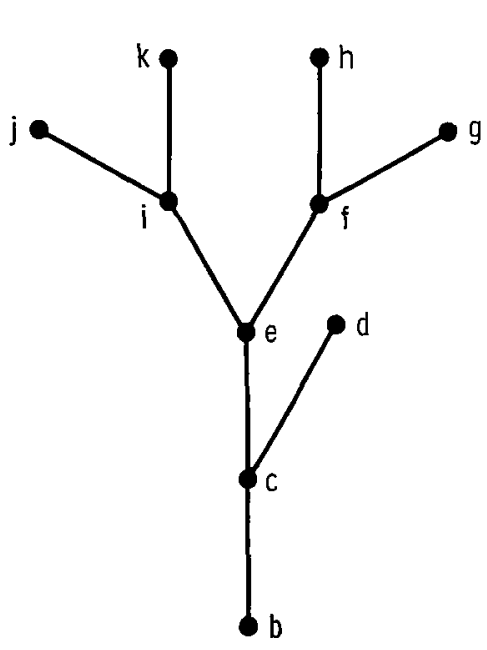

A

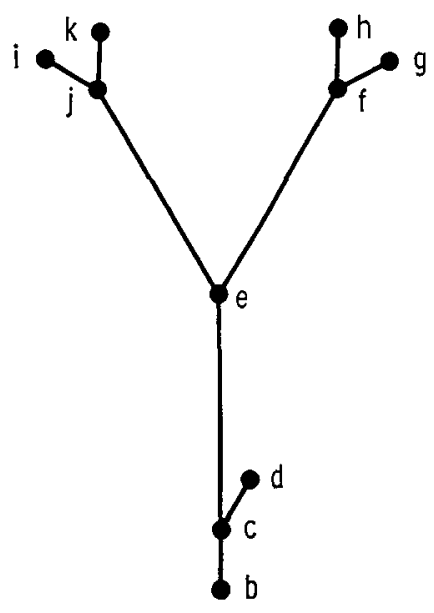

B

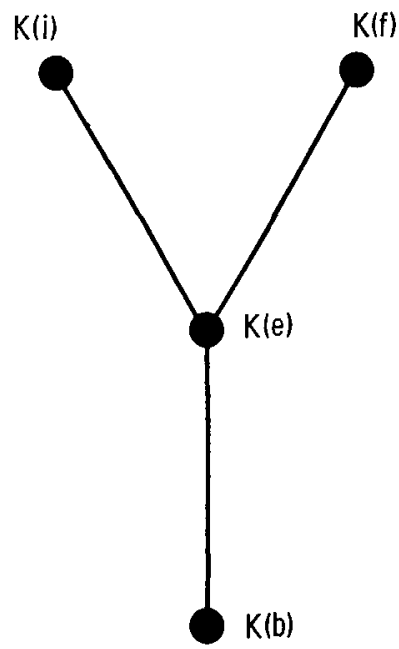

C

FIG. 3. The ideal relationship between the Hasse diagram for a set of evolutionary units and a character state tree. A. a Hasse diagram for a hypothetical set of evolutionary units; B. the same Hasse diagram as in (A) with the internodes between the equivalence classes defined by the character whose character state tree is shown in (C) stretched; $C$. a character state tree. The cladistic patterns for the equivalence classes (B) are the same as those for the character states $(\mathrm{C})$.

1. Each equivalence class of $E$ should contain its own most recent common ancestor (should contain a unique minimal element).

2. If a first $\mathrm{EU}$ is an ancestor of a second $\mathrm{EU}$, then the state of which the first is a member should be equal or primitive in the character state tree of $P$ to the state of which the second is a member.

3. If one character state is primitive to another in the character state tree of $P$, then the most recent common ancestor for the one state should be ancestral in A to the most recent common ancestor of the other.

If the relationship between $\mathrm{A}$ and $\mathrm{P}$ is ideal, then the partial order induced by $\mathrm{A}$ onto the subset of $S^{\prime}$ made up of the respective character-state minimal elements will be isomorphic to (the same as) P, whose Hasse diagram is the character state tree. Estabrook, Johnson \& McMorris (1975) prove as a theorem that a cladistic character with this relationship to $\mathrm{A}$ is a true cladistic character if the character state tree is true. 
Figure 3 illustrates the ideal relationship between the Hasse diagrams of $\mathrm{A}$ and $\mathrm{P}$ : each element of $\mathrm{P}$ corresponds to an element in $\mathrm{A}$ which is the minimal element in that state; thus $K(b)$ corresponds to $b, K(f)$ corresponds to $f$, etc. Note that if certain edges of the Hasse diagram of A are greatly stretched (i.e., those between EUs with different character states), the Hasse diagram for $\mathrm{P}$ results. A character is true if all the statements it makes about $\mathrm{A}$ are true; a false character makes statements about $\mathrm{A}$ which are false. The Hasse diagram for a true character and the Hasse diagram for A will have one fewer edge than there are states in the character, while a false character will specify edges of A which do not exist.

With the concept of an ideal cladistic character defined, a method of finding among the collection of real characters those characters which are most likely to be true is now needed. Under the assumption that there are only bifurcations in true evolutionary history, there are $\prod_{i=1}^{N-1}(2 i-1)$ possible estimates of evolutionary history for any set of $N$ EUs. This is a very large number for a study collection of even moderate size; even if many are highly unlikely, a large set of possible estimates still remains. Characters help to reduce the number of likely possibilities to a small set, which ideally is the one most likely estimate. Remember that each character divides the set of EUs into a set of equivalence classes, which for cladistic characters is a set of tree partial orders. Among all the possible evolutionary histories for a set of EUs only one is true. Each character in effect divides all the possible estimates into two groups. The first consists of those partial orders which, if any of them be true, result in the character's being true; the second contains those which, if any be true, result in the character's being false. Unfortunately, there is no technique for determining which characters are true. The concept of compatibility of characters (Le Quesne, 1969; Camin \& Sokal, 1965; Estabrook, 1972), however, determines which characters may be true. Two true characters may make different statements about A, but they never contradict each other. Two characters are compatible if it is logically possible for both to be true simultanecusly. Two characters are incompatible if they logically contradict each other; at least one of them is false. They may both be false, as two compatible characters may also be both false. Thus, given two partial orders defined by two different characters, compatibility means that the mathematical intersection of the two sets is non-empty; it contains all the estimates of evolutionary history which are logically possible and for which the estimates made by each character do not contradict each other. Usually this group of estimates is much smaller than for any character alone. An intersection which is empty implies that no estimate of evolutionary history allows both characters to be true simultaneously.

In order to test characters for compatibility, a character state tree must be created for each character used in the study. Short of knowing true evolutionary history, there is no sure way of doing this. Regardless of the complexity of the tree, the first step in creating a character state tree is to identify the primitive state represented in the study collection. Suggestions on how to do this are discussed by Sporne (1956), Wagner (1961), Kluge \& Farris (1969), Marx \& Rabb (1970) and Estabrook (1972), among others. The principal method used in this study is that of the ground plan or correlation. The problems of using this method are discussed by Colless (1969b) and Stebbins (1974). It is assumed that more primitive character states are more likely to be distributed throughout other groups similar to, and supposedly related to, the group under study (outgroups), are more likely to be 
widespread within the group under study than is any derived state, and are therefore likely to be associated in the same evolutionary units with the primitive states of other characters. For simple two-state characters, defining the primitive state fixes the character state tree. For characters with more than two states, the number of possible trees is only partially determined when a primitive state is identified. Construction of the character state tree must in this case be based on whatever evidence is available to predict the evolutionary relationship among the character states. Sometimes evidence from patterns found in other groups, such as the loss of parts or increased complexity of a structure, can be used. Often one must guess.

There are several reasons why many characters lead to incorrect historical inferences. Specimens may not all represent EUs; there may be hybrids, polyploids (Wagner, 1970), or specimens which are atypical because of disease, stress, or genetic abnormalities. A character may be constructed on the basis of false homology. Characters being compared may represent differences in life cycle or maturity. Finally, the character may show homoplasy; similar-appearing states may not share a common most recent ancestor.

What evidence is there that the set of characters is compatible because of a shared evolutionary history and is not just a chance result? Intuitively, it would be expected that few characters from a character set would be compatible over many EUs. Since there are many chances for errors in devising character state trees, many trees are probably false, and the number of incompatibilities in a data set is usually large. Statistical methods for testing the significance of the size of a set of compatible characters are currently unavailable, but it seems reasonable that finding a large set of mutually compatible characters is unlikely. My preliminary simulation experiments show that there are usually no compatibilities in a completely random data set, and it is only when some structure in the distribution of character states is imposed upon the EUs that compatibilities of about $3 \%$ of the total number of characters are found. The structure in real data sets is thought to be the result of an historical process. Thus the larger the set of mutually compatible characters (the larger the clique) found in a study, the greater the confidence that the compatibilities represent true history.

Fortunately, it is easy to determine the compatibilities in a set of characters by means of a computer. The cartesian product of two characters (a lattice containing a vertex for every possible combination of the character states of the two original characters) can be thought of as a new character (Estabrook, 1968, 1972). The cartesian product of two characters I and II, written I $\otimes I I$, is illustrated in Fig. 4 for two simple characters. If I and II are compatible, their cartesian product is a tree order; if incompatible, their cartesian product is not a tree order. Figure $4 \mathrm{~B}$ and $4 \mathrm{C}$ represent the cartesian products of compatible characters, while Fig. 4D represents the cartesian product of incompatible characters.

The characters which define the basic, or primary, phyletic lines of the group under study are called primary characters for that group. In the early analyses of a large group the relationships on the primary phyletic lines usually are only partially resolved. To resolve the relationships of these lines further, the analysis may be repeated on each line separately. Usually several additional characters are found to be compatible with the original primary characters. These additional characters are called secondary characters for the overall study or local primary characters for the line in which they are primary. This process may be repeated in smaller and smaller groups until all the local primary 


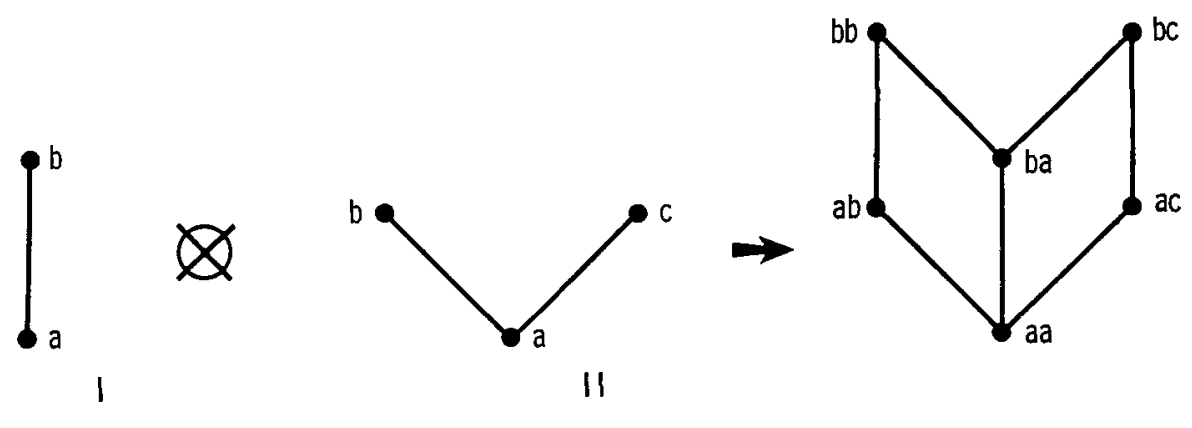

A

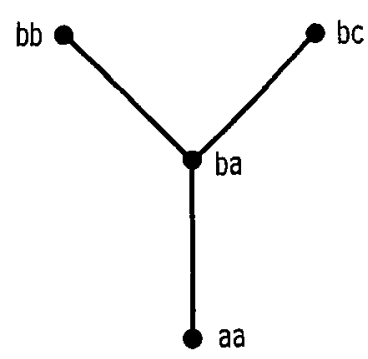

B

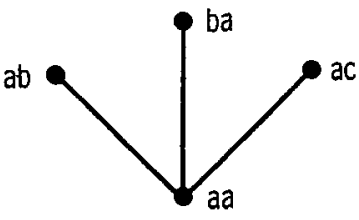

C

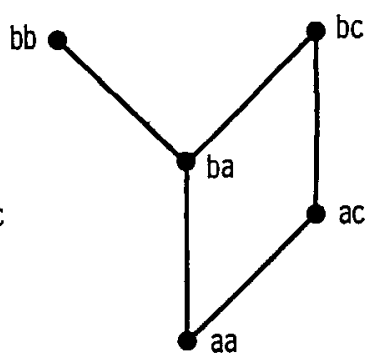

D

FIG. 4. Character compatibility. A. The cartesian product of two characters (I and II) whose character state trees are shown on the left is represented by the lattice on the right. If the nodes on this lattice which represent character state combinations not found in the study collection are discarded (along with unnecessary internodes) and the resulting diagram is a tree (B and $\mathrm{C}$ ), the characters are compatible; if the resulting figure is not a tree (D), the characters are incompatible.

characters have been found. As the size of the group becomes smaller, however, the chance that characters will be included randomly in the largest cliques increases.

Sometimes more than one largest clique is found in a given analysis. Either all the largest cliques may be accepted as defining equally probable estimates of relationships, or their intersections (set of characters included in all the cliques) may be accepted as defining the best estimate of relationships, or a choice may be made among them. A choice among the cliques may be made only if a choice among the conflicting characters can be made. Characters in the clique which defined the monophyletic line are usually more reliable, since they have already passed a more rigorous test. Occasionally, only a clique smaller than the largest clique can pass this test. Characters which make more complex statements about relationships, either because of the shape of their character state trees or because of the distribution of their states over the EUs, may be chosen over characters which make simple statements about relationships because it is less probable that they are included in a largest clique by chance alone. A character whose biology is well known may be chosen over a character whose biology is poorly known if the biological knowledge supports the character state trees. In some cases the conflicting characters may be involved only in the 
branching patterns at the tips of some branches and thus do not affect the major branching patterns of the overall tree. In those cases a decision on the value of a character may be postponed until the smaller monophyletic groups have been analysed.

Some characters may be rejected in a compatibility analysis because they are homoplastic on a restricted part of the phylogenetic tree. If the instances of homoplasy for some characters have taken place early in the history of the group, these characters may be true cladistic characters for some of the branches of the phylogenetic tree. The technique of finding locally compatible characters in smaller and smaller monophyletic groups identifies such characters. If the instances of homoplasy have occurred late in the history of the group, however, some characters may be rejected by all the compatibility analyses in which they are examined. In instances where the early branching patterns of the tree being reconstructed are poorly resolved, the latter type of characters may be identified and used to increase the resolution of the tree.

The following procedure may be used. After the smaller monophyletic groups have been identified and analysed, the EUs in each group which are least derived are identified, or the character states of the most recent common ancestor of the group are estimated. A compatibility analysis using only the "more conservative" EUs, or the reconstructed ancestors, is then made to find additional characters compatible with the original clique. This procedure has the effect of removing instances of homoplasy which occur at the tips of the branches and objectively identifies additional characters for reconstructing the early branching pattern of the phylogenetic tree. Since they are based on characters with known homoplasy, the transitions defined in this manner are less certain than those identified in the original analysis of the group. While this method may be used to extract the maximum amount of information from a particular data set, it is inferior to the addition of new characters to the data set.

The characters found to be true in a study may always be tested against new characters. This is particularly important for analysing small monophyletic groups in which only a few characters vary, since such analyses usually yield several largest cliques. Because it is often impossible to decide among these cliques, it is best to increase the character set and perform a new analysis. Often many characters which could not be coded satisfactorily for the entire study collection can be introduced at this stage. Plumage characters in birds are an example.

\section{Characters}

Descriptions and character tree codings are given below for the 70 characters used in this study. The majority (63) are characters of the skeleton, whose homologies were determined according to their relative position on the skeleton. (See Jardine (1969) for a formal method.) Since the avian skeleton is quite uniform throughout the class, homology based on relative position is probably the same as the evolutionary homology which would be obtained if the phylogeny of each structure could be followed from a structure found in the most recent common ancestor of the birds to the structures found in living species (Simpson, 1961). This assumption might fail in the case of some of the complex structures of the skull and hypotarsus, however, especially since the ontogeny of these structures has been studied in only a few birds (Jollie, 1957; Cracraft, 1968). Character codings for the 227 species studied are given in Table I. 


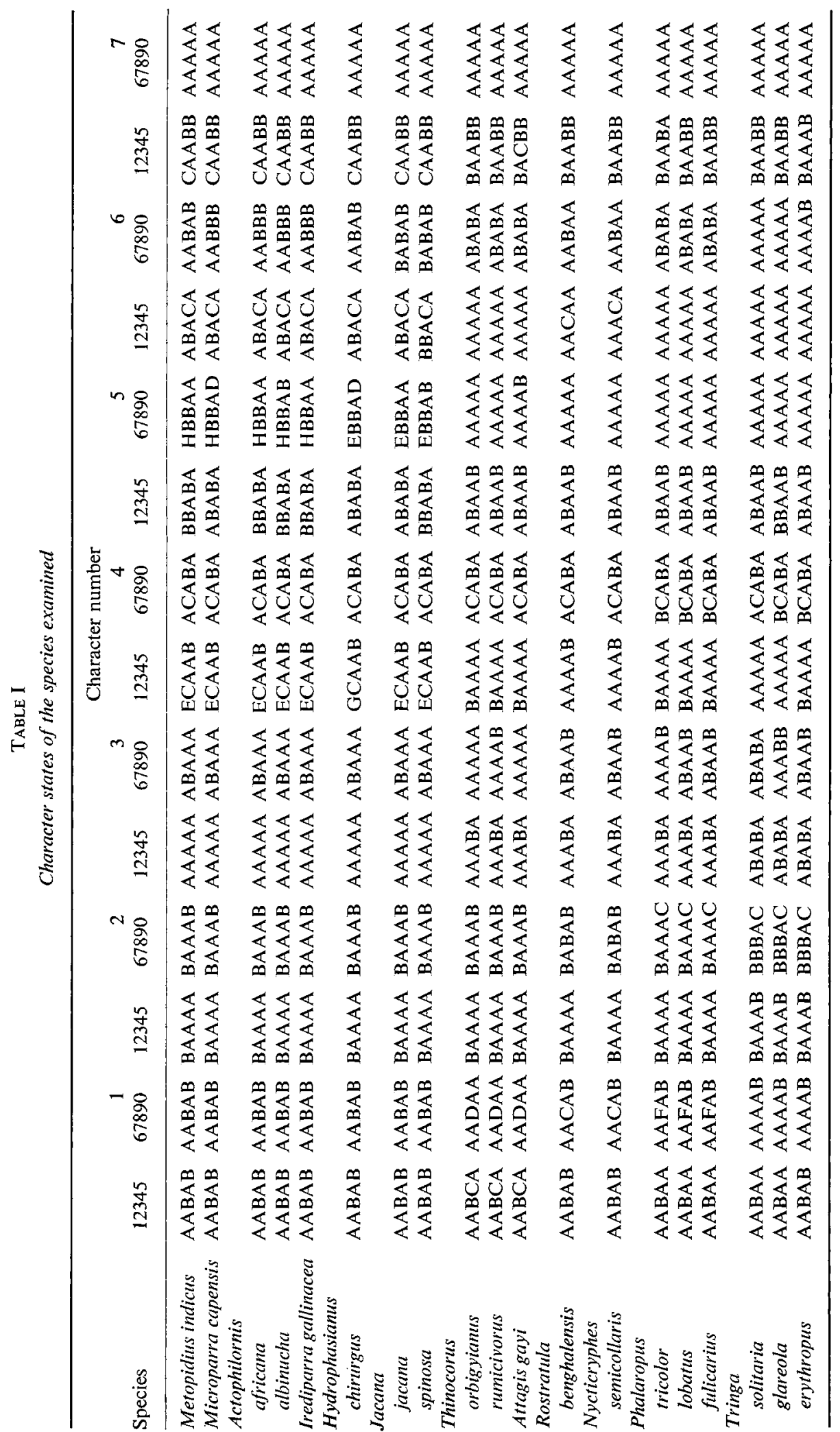




\begin{tabular}{|c|c|c|c|c|c|c|}
\hline - & 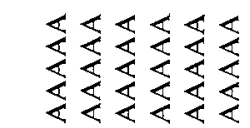 & $\sum_{i}^{\infty}$ & 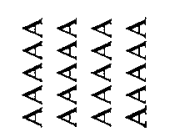 & 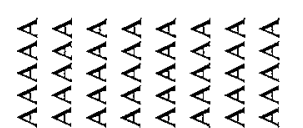 & $\begin{array}{l}<\leqslant \leqslant \\
<\leqslant \leqslant\end{array}$ & 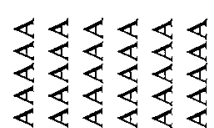 \\
\hline 离 & 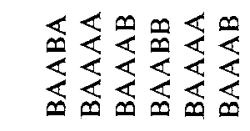 & $\sum_{\infty}^{\infty}$ & 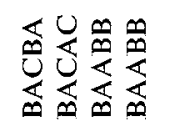 & 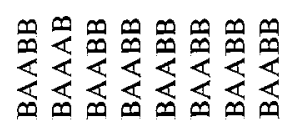 & 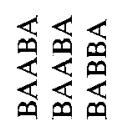 & 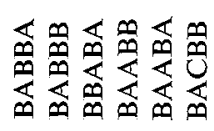 \\
\hline 용 & 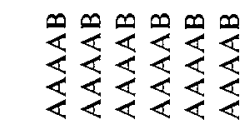 & $\underset{\infty}{\infty}$ & 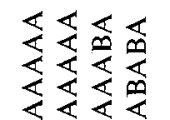 & 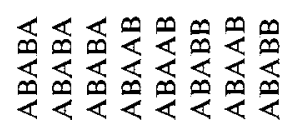 & 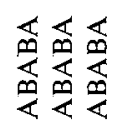 & 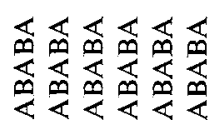 \\
\hline 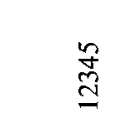 & 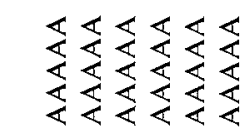 & $\sum_{\frac{1}{4}}^{\infty}$ & 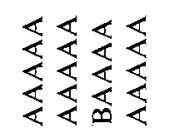 & 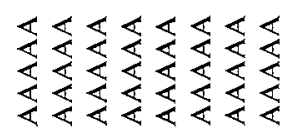 & 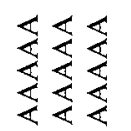 & 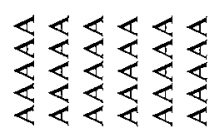 \\
\hline n $\frac{8}{\infty}$ & 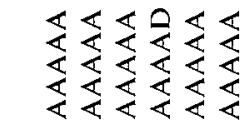 & $\frac{\pi}{4}$ & 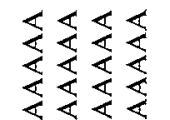 & 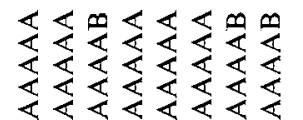 & $\sum_{i}^{\infty} \leqslant \frac{1}{<}<\frac{\pi}{4}$ & 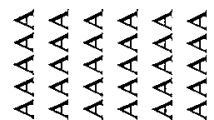 \\
\hline 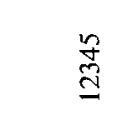 & 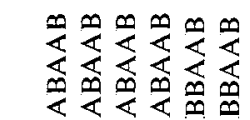 & $\sum_{\infty}^{\infty}$ & 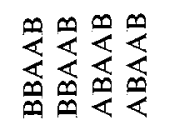 & 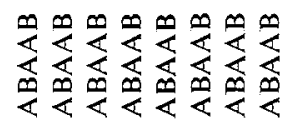 & $\sum_{\infty}^{\infty}<\frac{\infty}{<}<\frac{\infty}{<}$ & 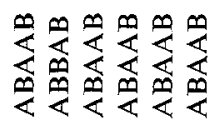 \\
\hline 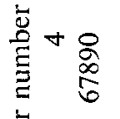 & 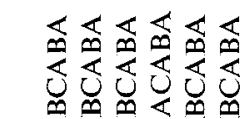 & 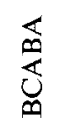 & 瓜芯希吕 & 希希希希希希 & 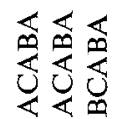 & 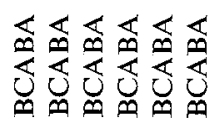 \\
\hline 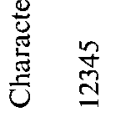 & 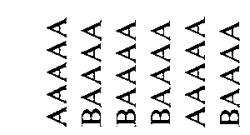 & $\frac{\pi}{\frac{1}{4}}$ & 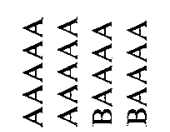 & 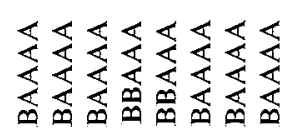 & $\sum_{\infty} \leqslant \frac{1}{\infty} \leqslant$ & 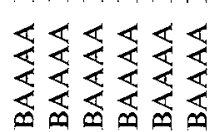 \\
\hline 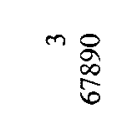 & 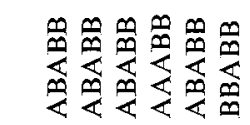 & 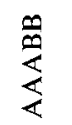 & 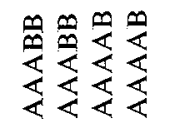 & 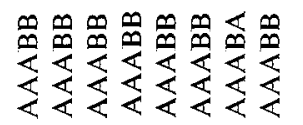 & $\sum_{i}^{\infty} \leqslant \frac{1}{<}<$ & 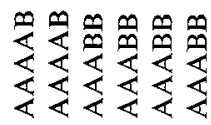 \\
\hline$\stackrel{n}{\stackrel{n}{7}}$ & 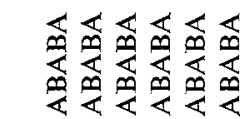 & 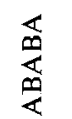 & 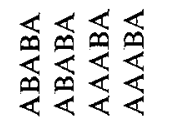 & 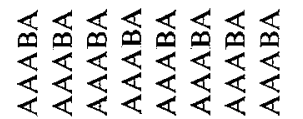 & 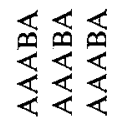 & 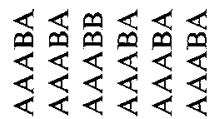 \\
\hline N & 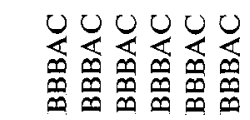 & 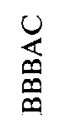 & 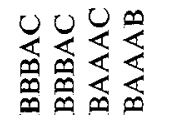 & 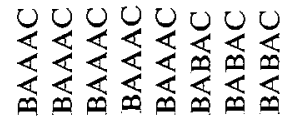 & 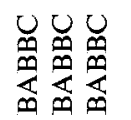 & 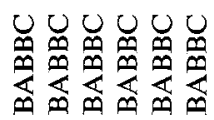 \\
\hline$\stackrel{n}{ \pm}$ & $\sum_{\infty}^{\infty} \sum_{\infty}^{\infty} \sum_{\infty}^{\infty} \frac{\infty}{<} \sum_{\infty}^{\infty} \frac{\infty}{d}$ & $\sum_{\substack{\infty \\
\infty}}^{\infty}$ & 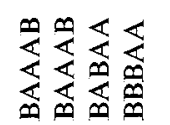 & 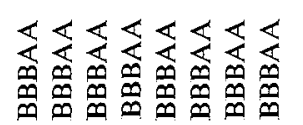 & $\frac{\pi}{4} \leqslant \frac{1}{4}$ & 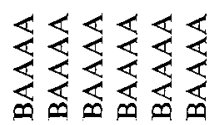 \\
\hline 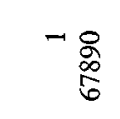 & 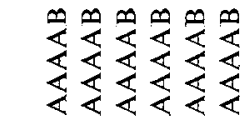 & $\sum_{i}^{\infty}$ & 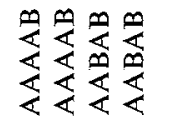 & 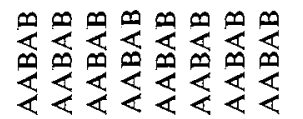 & 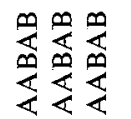 & 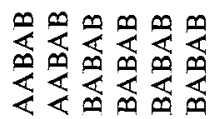 \\
\hline$\stackrel{n}{\stackrel{n}{d}}$ & 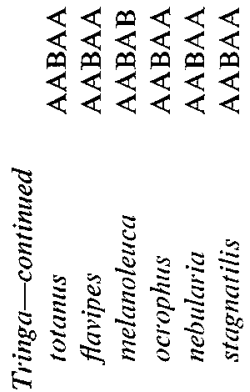 & $\frac{⿱ 亠}{4}$ & 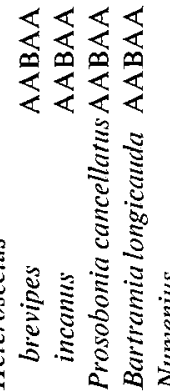 & 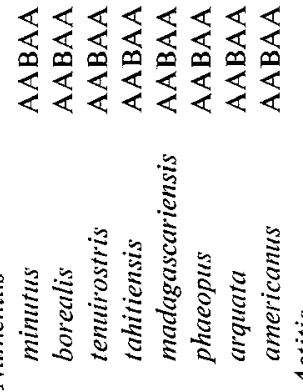 & 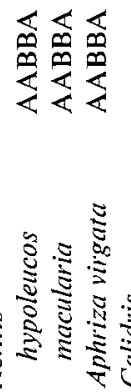 & 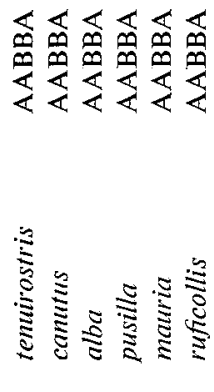 \\
\hline
\end{tabular}




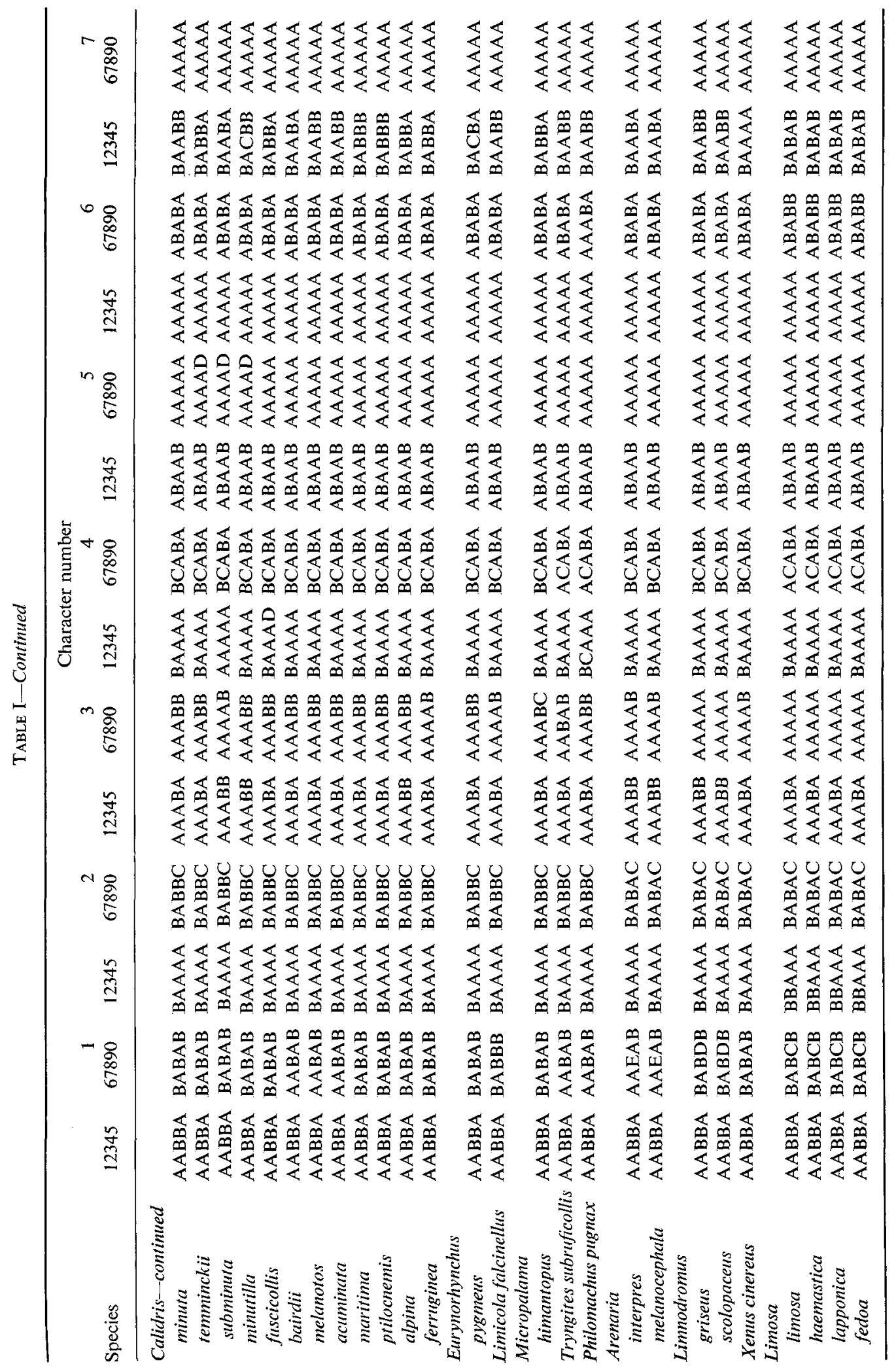




\begin{tabular}{|c|c|c|c|c|c|}
\hline $\begin{array}{r}-8 \\
\stackrel{8}{6} \\
6\end{array}$ & $\sum_{i}^{\infty} \sum_{i}^{\infty}$ & 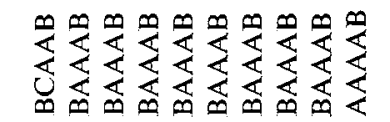 & 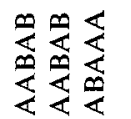 & 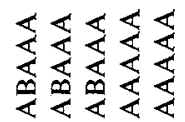 & 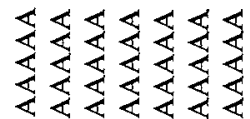 \\
\hline 尔 & $\sum_{\infty}^{\infty} \frac{\infty}{\infty}$ & 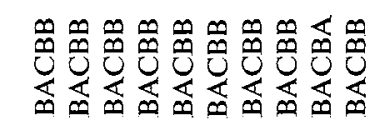 & 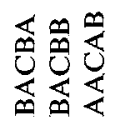 & 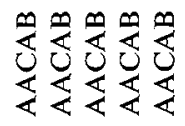 & 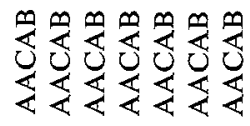 \\
\hline 용 & $\sum_{i}^{\infty} \frac{\pi}{\infty}$ & 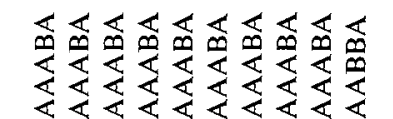 & 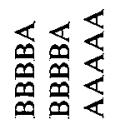 & 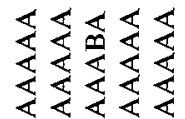 & 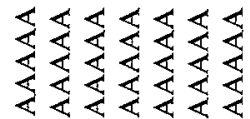 \\
\hline 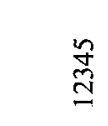 & $\sum_{i}^{\infty} \frac{\pi}{4}$ & 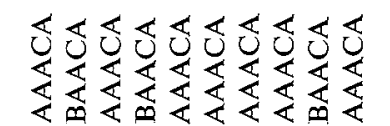 & 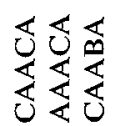 & 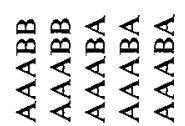 & 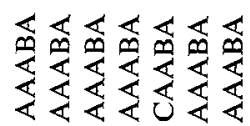 \\
\hline n $\frac{8}{8}$ & $\sum_{i} \frac{1}{<}$ & 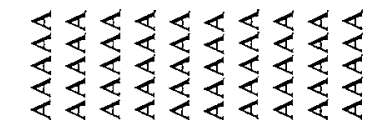 & 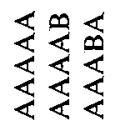 & 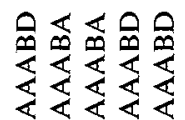 & 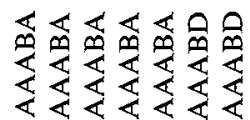 \\
\hline 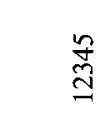 & $\sum_{\substack{\infty \\
<}}^{\infty} \frac{\pi}{<}$ & 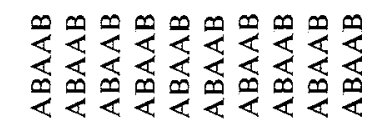 & 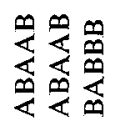 & 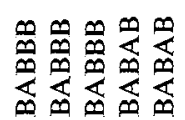 & 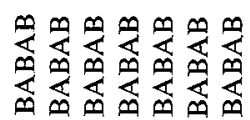 \\
\hline 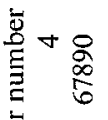 & 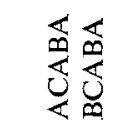 & 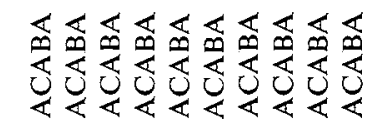 & 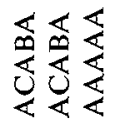 & 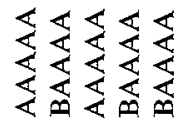 & 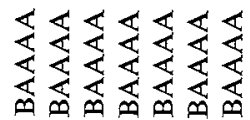 \\
\hline$\stackrel{n}{\stackrel{n}{3}}$ & $\sum_{\infty}^{\infty} \frac{\pi}{4}$ & 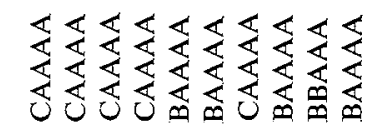 & $\frac{9}{4} \leqslant \frac{1}{4}$ & 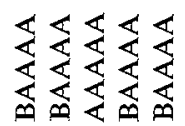 & 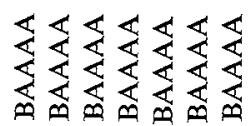 \\
\hline 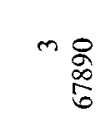 & $\sum_{\substack{1 \\
<}}^{\infty} \frac{1}{2}$ & 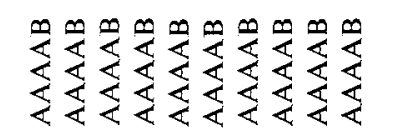 & $\sum_{i}^{\infty} \sum \sum$ & 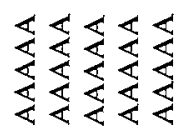 & 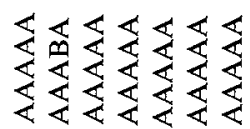 \\
\hline 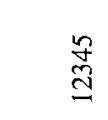 & 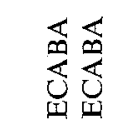 & 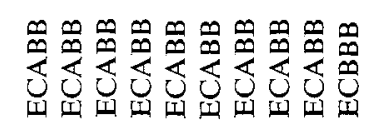 & 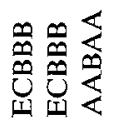 & 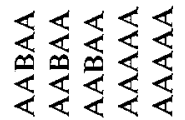 & 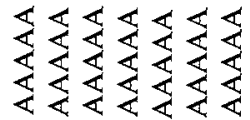 \\
\hline $\begin{array}{r}N \& \\
\underset{0}{0}\end{array}$ & $\underset{\infty}{\infty} \underset{\infty}{\infty}$ & 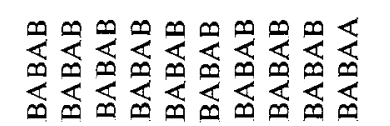 & $\begin{array}{l}\ll \\
\ll\end{array} \frac{1}{\infty}$ & 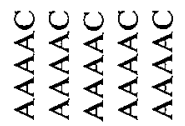 & 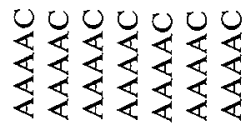 \\
\hline 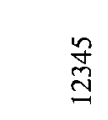 & $\sum_{0}^{\infty} \sum_{\infty}^{\infty}$ & 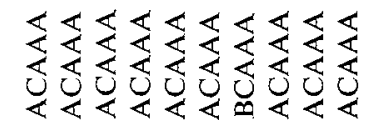 & $\sum_{\infty}^{\infty} \sum_{\infty}^{\infty} \sum_{\infty}^{\infty}$ & 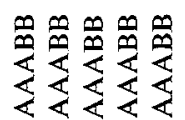 & 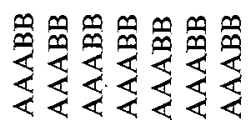 \\
\hline$-\frac{8}{2}$ & $\sum_{\substack{\infty \\
\infty}}^{\infty}$ & 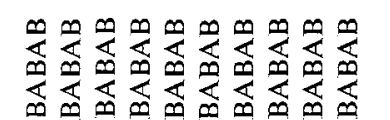 & $\sum_{\infty}^{\infty} \sum_{\infty}^{\infty} \sum$ & 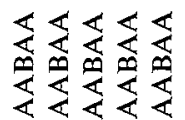 & 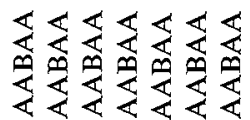 \\
\hline 先 & 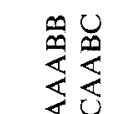 & 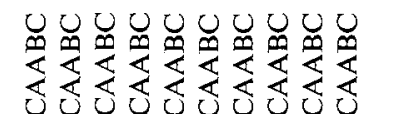 & 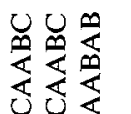 & $\begin{array}{l}0 \\
0 \\
0\end{array}$ & $\begin{array}{l}\infty \\
\infty \\
\infty\end{array}$ \\
\hline 䢘 & 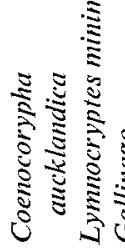 & 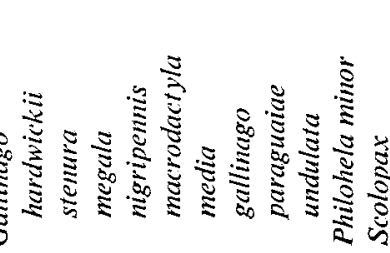 & $\Xi$ & 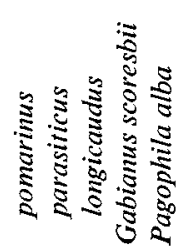 & 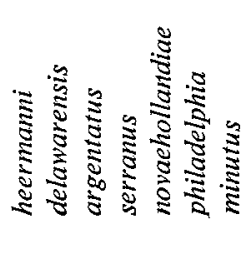 \\
\hline
\end{tabular}




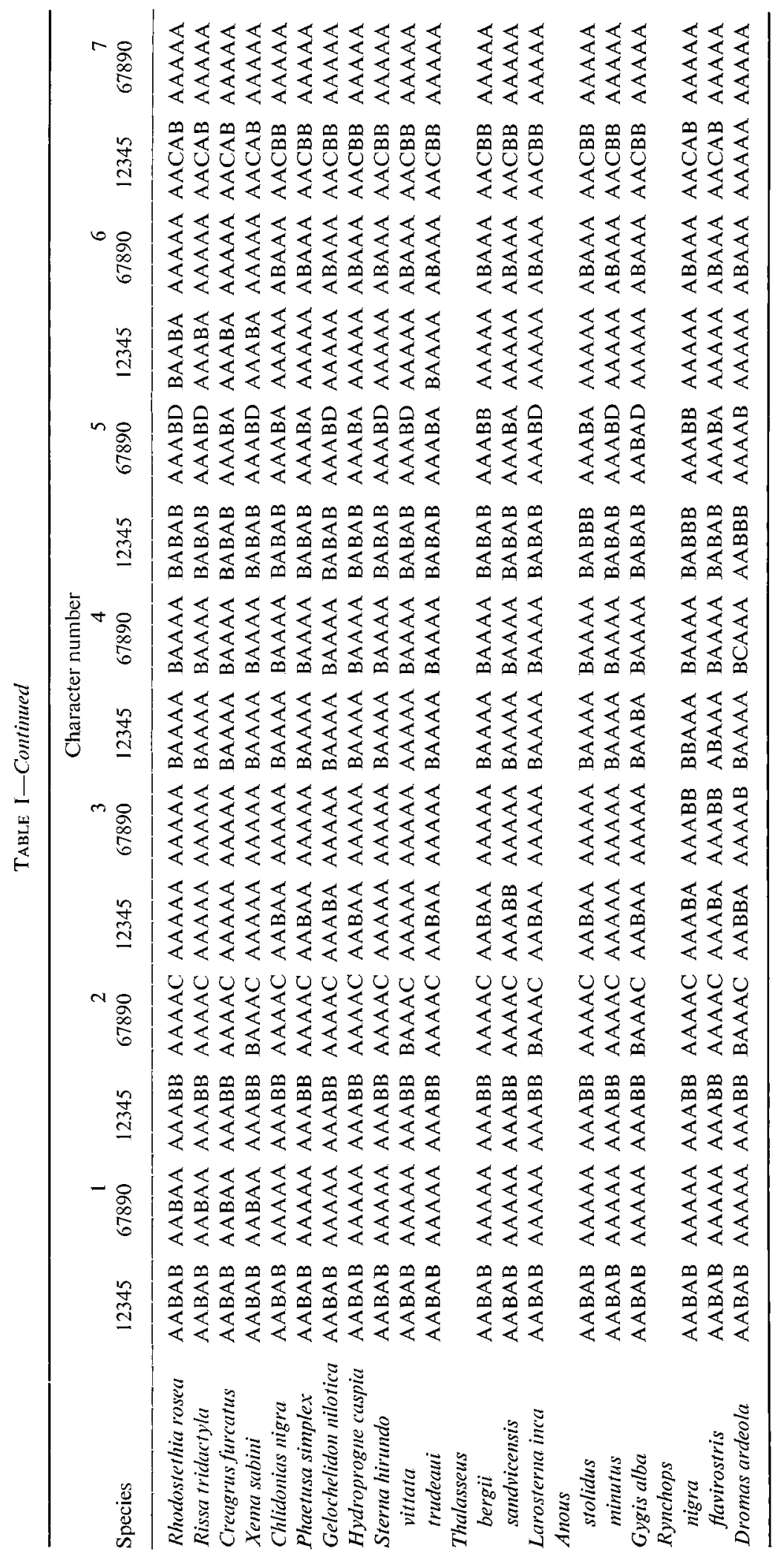




\begin{tabular}{|c|c|c|c|c|c|}
\hline - & $\sum_{0}^{\infty}$ & 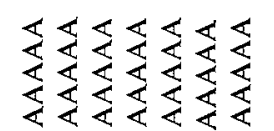 & $\sum_{\infty}^{\infty} \sum \sum$ & 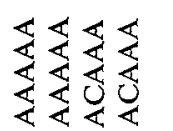 & 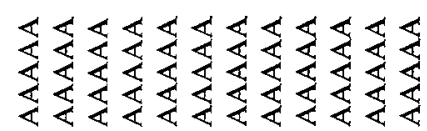 \\
\hline 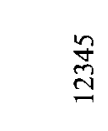 & $\sum_{\infty}^{\frac{1}{\infty}}$ & 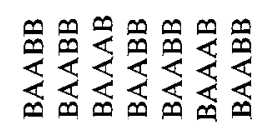 & $\sum_{\substack{\infty \\
\infty}}^{\infty} \sum_{\infty}^{\infty}$ & 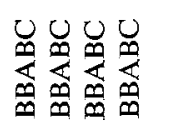 & 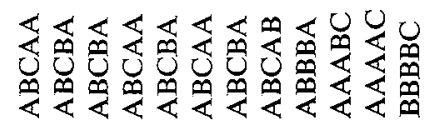 \\
\hline 용 & $\sum_{\substack{\infty \\
<}}^{\infty}$ & 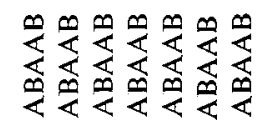 & 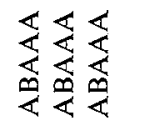 & 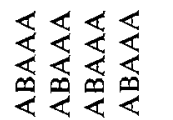 & 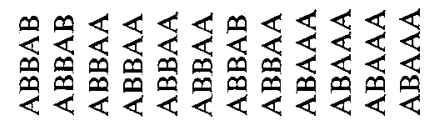 \\
\hline 告 & 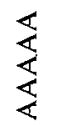 & 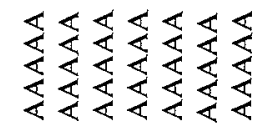 & 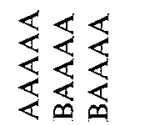 & $\sum_{<} \leq \frac{1}{<}<\frac{1}{<}$ & 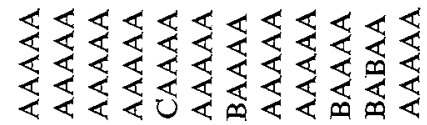 \\
\hline n $\frac{8}{\infty}$ & $\sum_{i}^{\infty}$ & 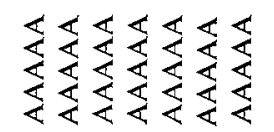 & 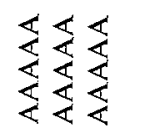 & 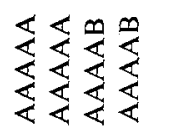 & 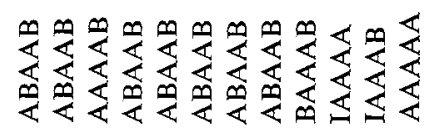 \\
\hline 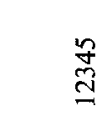 & 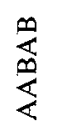 & 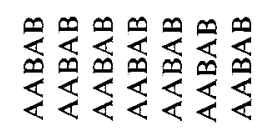 & 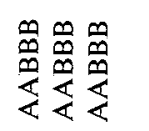 & 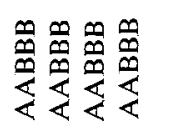 & 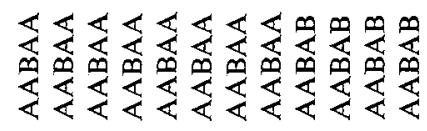 \\
\hline 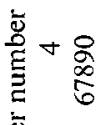 & 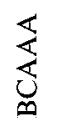 & 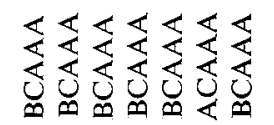 & $\sum_{\infty}^{\infty} \sum_{\infty}^{4} \sum_{\infty}^{1}$ & 㫐志芯导 & 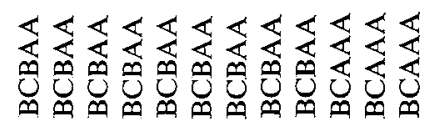 \\
\hline 总 & $\sum_{i}^{\infty}$ & 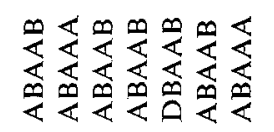 & 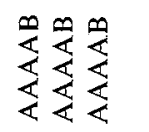 & 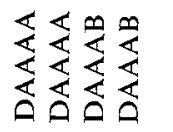 & 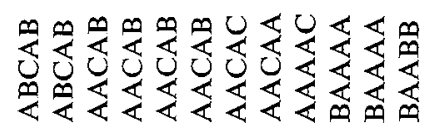 \\
\hline m & $\sum_{i}^{\infty}$ & 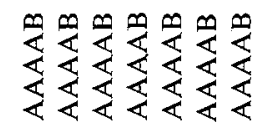 & 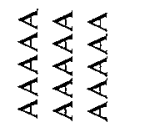 & 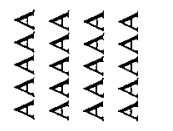 & 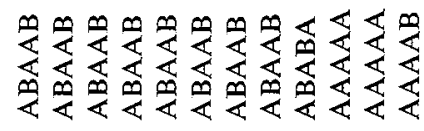 \\
\hline 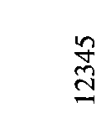 & 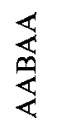 & 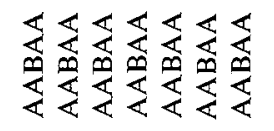 & 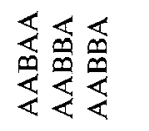 & 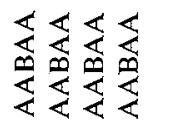 & 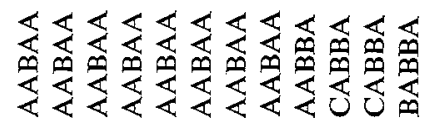 \\
\hline 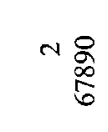 & 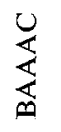 & 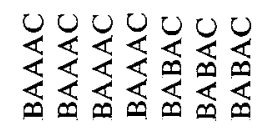 & $\sum_{\infty}^{\infty} \sum_{\infty}^{\infty} \frac{1}{\infty}$ & 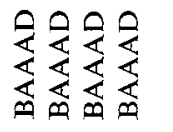 & 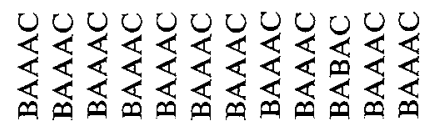 \\
\hline 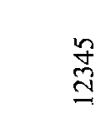 & 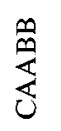 & 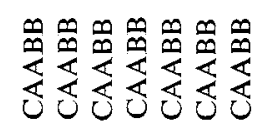 & 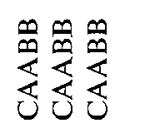 & 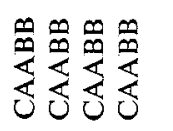 & 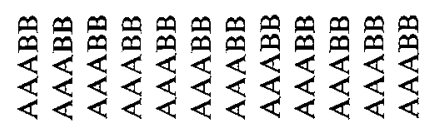 \\
\hline$\frac{\infty}{6}$ & $\sum_{\substack{\infty \\
\ll}}^{\infty}$ & 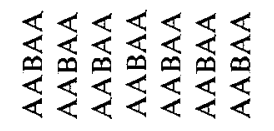 & 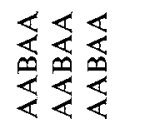 & 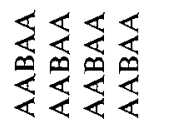 & 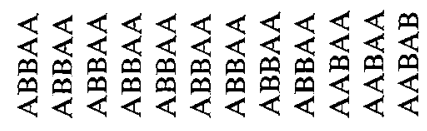 \\
\hline$\stackrel{⿱}{\text { తે }}$ & $\sum_{i}^{\infty}$ & 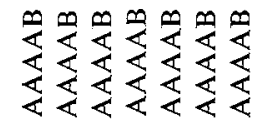 & $\sum \sum \sum \sum$ & 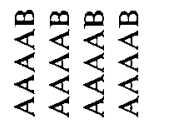 & 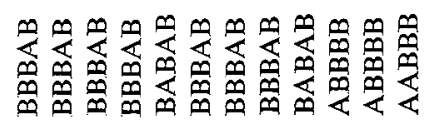 \\
\hline$\frac{\mathscr{Z}}{\mathscr{E}}$ & 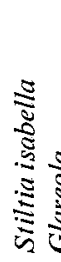 & 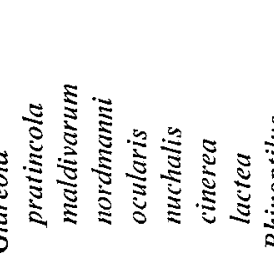 & $=\stackrel{5}{5}$ & 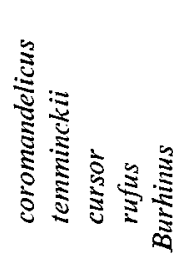 & 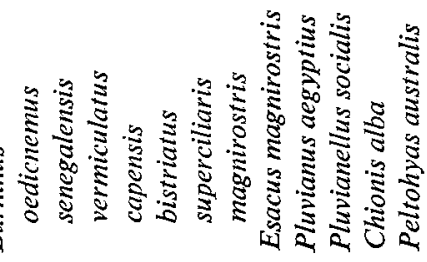 \\
\hline
\end{tabular}




\begin{tabular}{|c|c|c|c|c|c|c|c|c|}
\hline r。 & 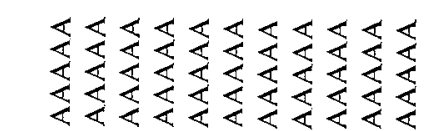 & $\sum_{i \leq 1} \leq \frac{1}{<}$ & 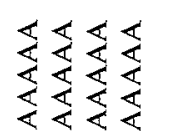 & 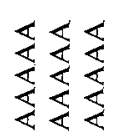 & $\sum_{i}^{\infty} \frac{1}{4}$ & $\sum_{i}^{\infty}$ & $\sum_{i}^{\infty} \leqslant$ & $\sum_{\infty}^{\infty}$ \\
\hline 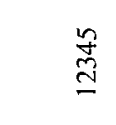 & 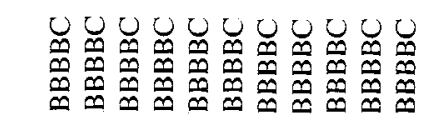 & 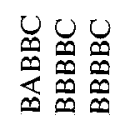 & 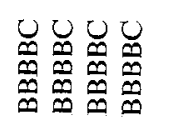 & 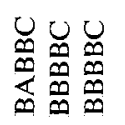 & 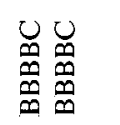 & $\begin{array}{l}0 \\
\infty \\
\infty \\
\infty \\
\infty\end{array}$ & 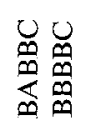 & 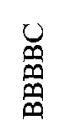 \\
\hline 용 & 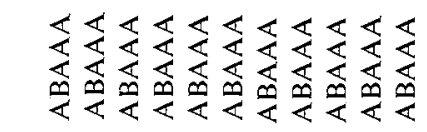 & 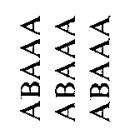 & 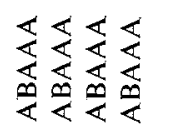 & 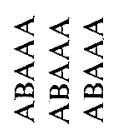 & 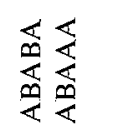 & 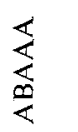 & $\sum_{\infty}^{\infty} \sum_{\infty}^{\infty}$ & $\sum_{\infty}^{\infty}$ \\
\hline $\begin{array}{l}\text { 年 } \\
\text { ḋ }\end{array}$ & 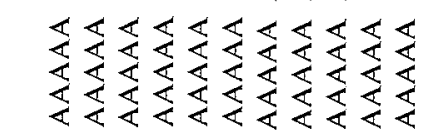 & 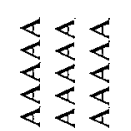 & 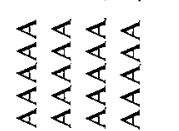 & 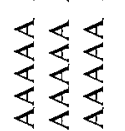 & $\sum_{\frac{1}{4}}^{4}$ & $\sum_{<}^{\infty}$ & $\sum \sum$ & $\sum_{i}^{\infty}$ \\
\hline in $\underset{\substack{0 \\
6}}{8}$ & 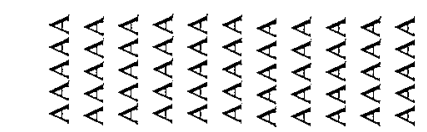 & $\frac{1}{<}<\frac{1}{<}$ & 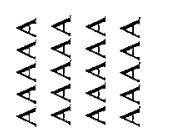 & 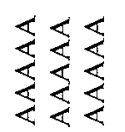 & $\sum_{i}^{\infty} \frac{1}{4}$ & $\sum_{i}^{\infty}$ & $\sum \frac{1}{4}$ & $\sum_{\substack{1 \\
4}}^{\infty}$ \\
\hline 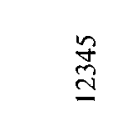 & 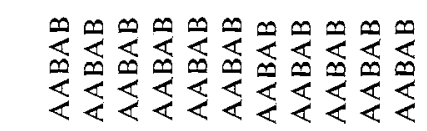 & $\begin{array}{l}\infty \\
\mathbb{\infty} \infty \\
<\frac{\infty}{<}\end{array}$ & 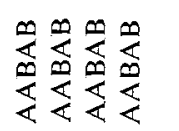 & 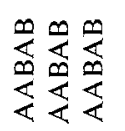 & 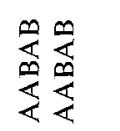 & $\sum_{\substack{\infty \\
<}}^{\infty}$ & $\begin{array}{l}\infty \\
\sum \\
\infty\end{array}$ & $\underset{\substack{\infty \\
\ll}}{\infty}$ \\
\hline 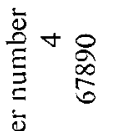 & 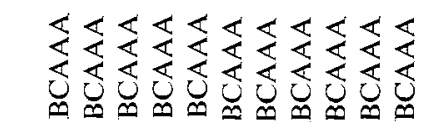 & 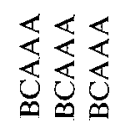 & $\sum_{\infty} \sum_{\infty} \sum_{\infty} \sum_{\infty} \sum_{\infty}$ & 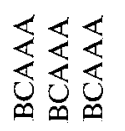 & 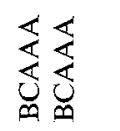 & $\sum_{\infty}^{\infty}$ & 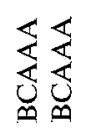 & 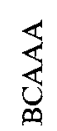 \\
\hline 莺 & 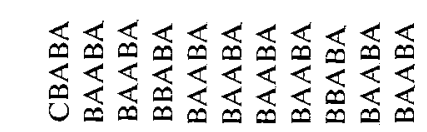 & 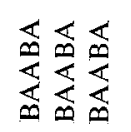 & 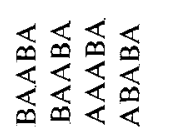 & 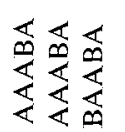 & 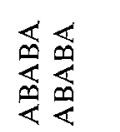 & 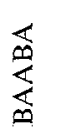 & $\sum_{\infty}^{\infty} \frac{\pi}{4}$ & 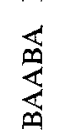 \\
\hline 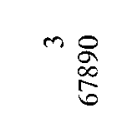 & 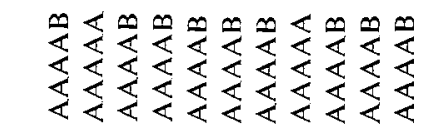 & 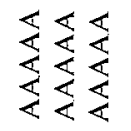 & 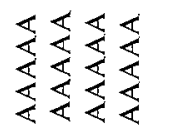 & 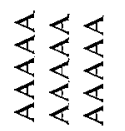 & $\frac{\infty}{4} \frac{\infty}{4}$ & $\sum_{<}^{\infty}$ & $\sum_{<}^{\infty} \frac{\infty}{<}$ & $\sum_{i}^{\infty}$ \\
\hline 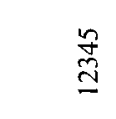 & 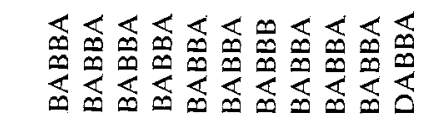 & 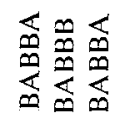 & 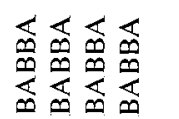 & 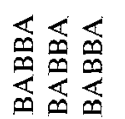 & $\underset{m}{\frac{\infty}{m}} \frac{\infty}{\infty}$ & $\frac{m}{\infty}$ & 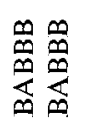 & 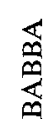 \\
\hline 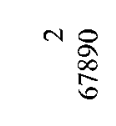 & 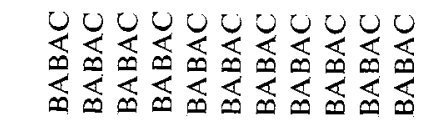 & 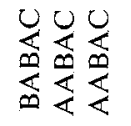 & 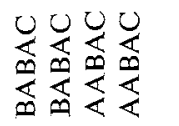 & 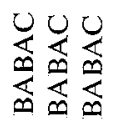 & $\underset{\substack{u \\
\infty}}{\substack{\infty \\
\infty}}$ & 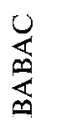 & 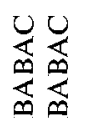 & 这 \\
\hline$\stackrel{n}{\stackrel{n}{a}}$ & 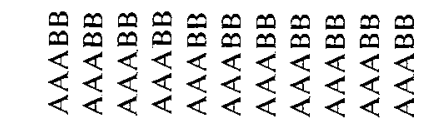 & $\begin{array}{l}\infty \\
\sum\end{array} \frac{\infty}{\infty} \frac{\infty}{<}$ & 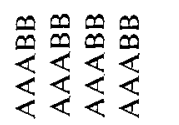 & 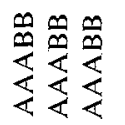 & 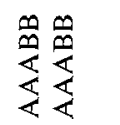 & 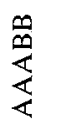 & $\sum_{i}^{\infty} \frac{\infty}{\infty}$ & $\frac{\infty}{\infty}$ \\
\hline $\begin{array}{r}-8 \\
\stackrel{8}{6} \\
6\end{array}$ & 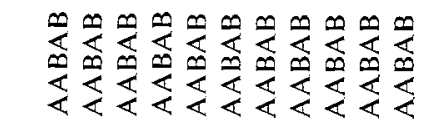 & 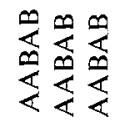 & 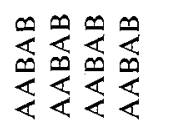 & 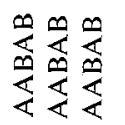 & $\sum_{\substack{\infty \\
<}}^{\infty}$ & $\sum_{\infty}^{\infty}$ & $\begin{array}{l}\infty \\
\infty \\
\infty\end{array}$ & $\frac{\infty}{4}$ \\
\hline$\stackrel{n}{\stackrel{n}{d}}$ & 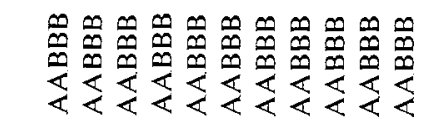 & 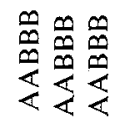 & 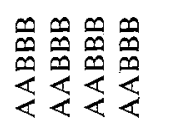 & 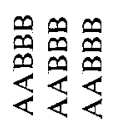 & 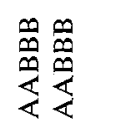 & 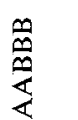 & $\frac{\infty}{\infty} \frac{\infty}{\infty} \frac{\infty}{\infty}$ & 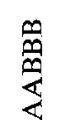 \\
\hline.$\frac{0}{8}$ & 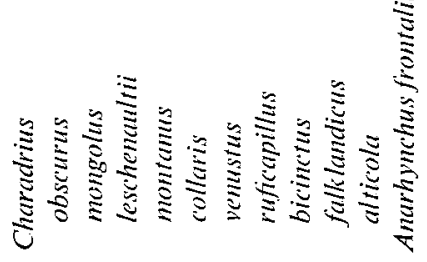 & $\Xi$ & $\Xi$ & 5 & 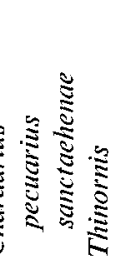 & 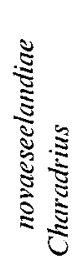 & 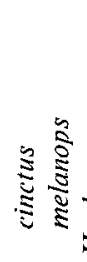 & \\
\hline
\end{tabular}




\begin{tabular}{|c|c|c|c|}
\hline r & $\sum_{<}^{\infty}$ & 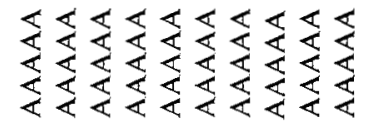 & 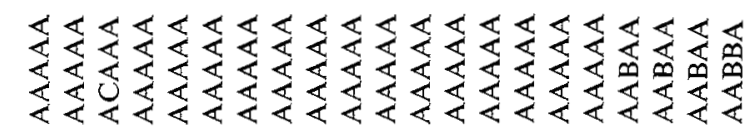 \\
\hline$\stackrel{\stackrel{n}{a}}{\underline{a}}$ & 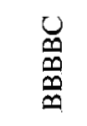 & 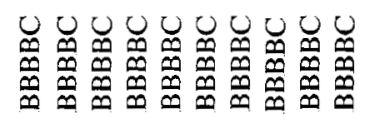 & 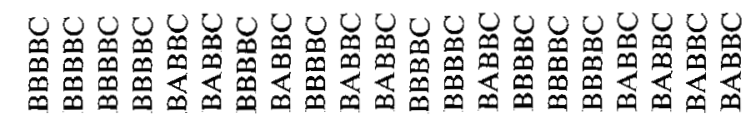 \\
\hline ○: & 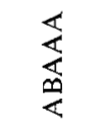 & 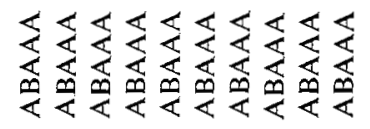 & 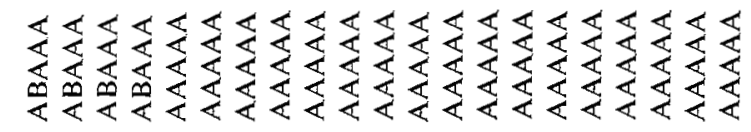 \\
\hline 嵒 & $\sum_{<}^{\infty}$ & 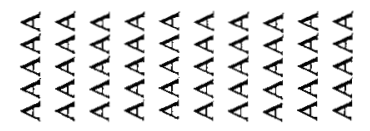 & 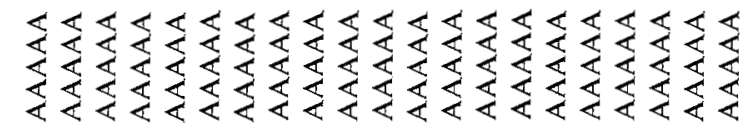 \\
\hline 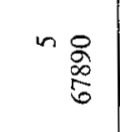 & $\sum$ & 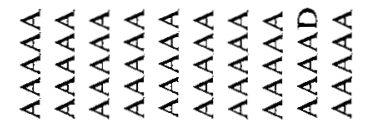 & 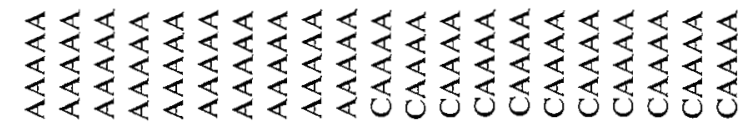 \\
\hline 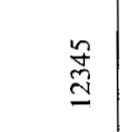 & $\underset{\substack{\infty \\
0}}{\infty}$ & 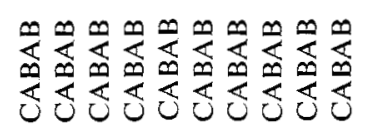 & 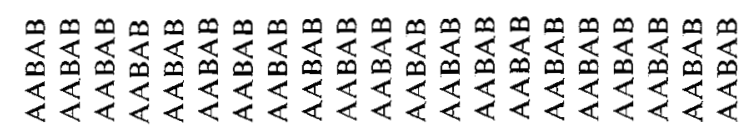 \\
\hline 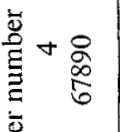 & $\sum_{\infty}^{\infty}$ & 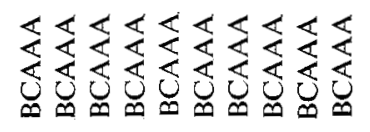 & 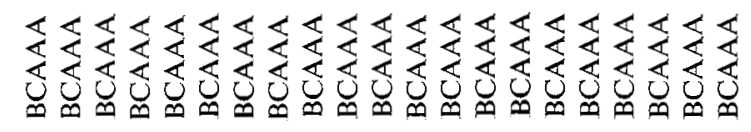 \\
\hline 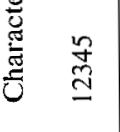 & 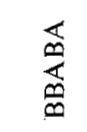 & 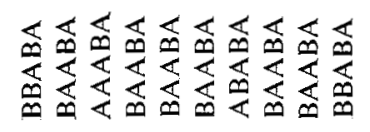 & 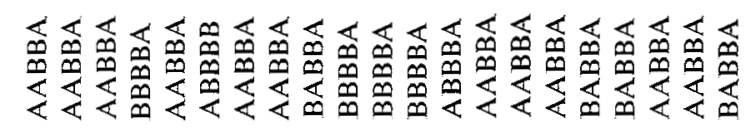 \\
\hline$m \frac{8}{\substack{\infty \\
6}}$ & $\sum_{i}^{\infty}$ & 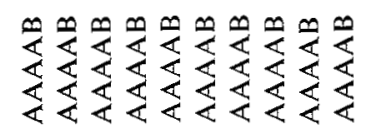 & 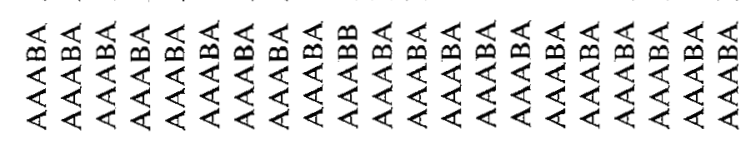 \\
\hline 梁 & 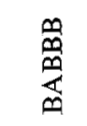 & 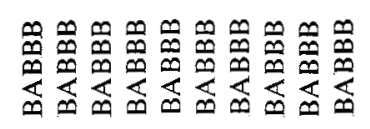 & 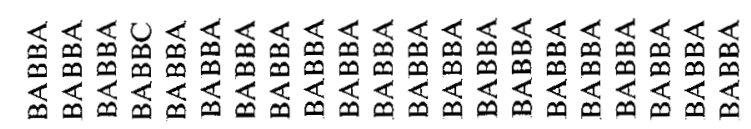 \\
\hline N & 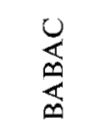 & 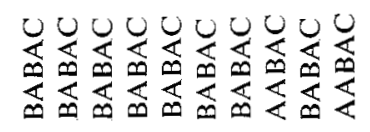 & 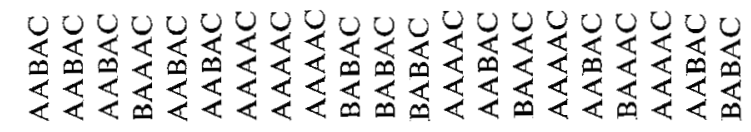 \\
\hline 守 & $\sum_{\substack{\infty \\
\infty}}^{\infty}$ & 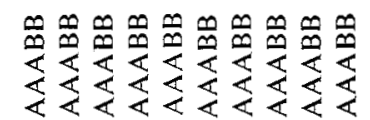 & 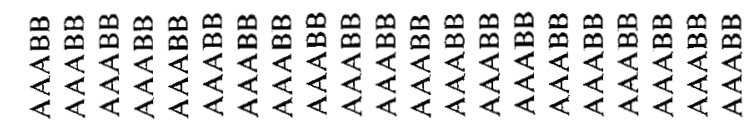 \\
\hline$\frac{8}{\infty}$ & 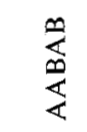 & 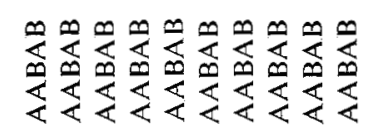 & 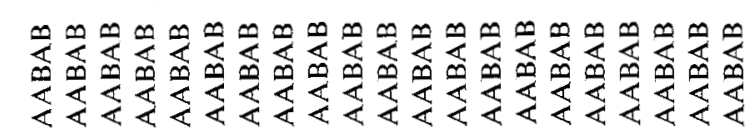 \\
\hline 粱 & $\underset{\infty}{\infty}$ & 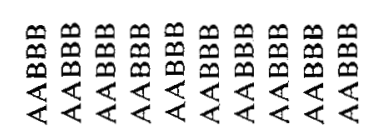 & 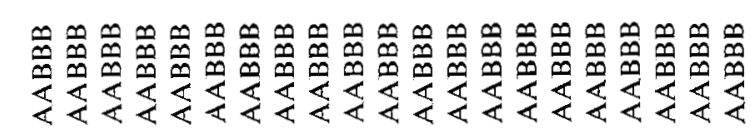 \\
\hline. & 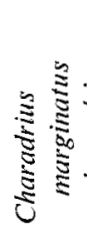 & 气气 & 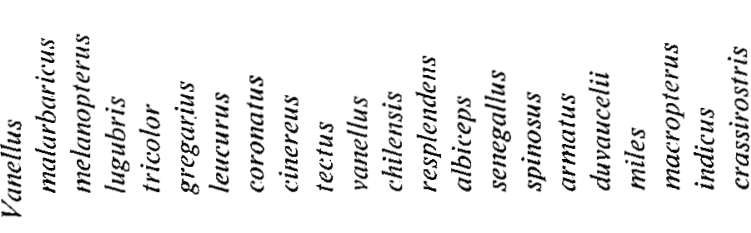 \\
\hline
\end{tabular}




\begin{tabular}{|c|c|c|c|c|c|c|}
\hline r & 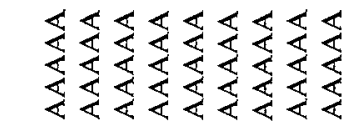 & $\sum_{i}^{\infty}$ & 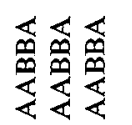 & 商 & 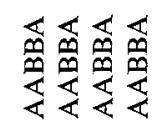 & 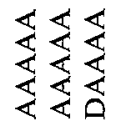 \\
\hline$\stackrel{\tilde{v}}{\stackrel{n}{\Xi}}$ & 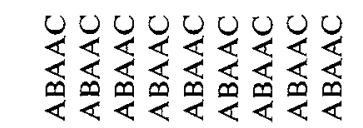 & $\sum_{\substack{u \\
<}}^{0}$ & 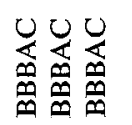 & $\begin{array}{l}\frac{1}{1} \\
\frac{\infty}{0} \\
\frac{0}{m}\end{array}$ & 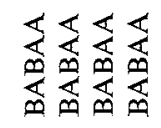 & 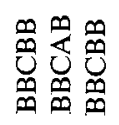 \\
\hline 용 & 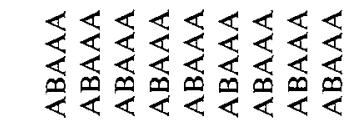 & $\sum$ & $\sum_{<}^{\infty} \frac{\infty}{4} \frac{1}{4}$ & $\sum_{i}^{\infty}$ & $\sum_{i} \leq \sum \leq \leqslant$ & 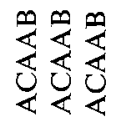 \\
\hline$\stackrel{\tilde{\Xi}}{\underline{\Xi}}$ & 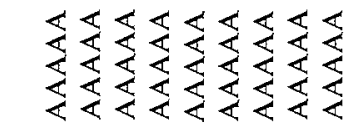 & $\sum$ & $\begin{array}{l}<\leqslant< \\
<\leqslant<\end{array}$ & $\sum_{\frac{1}{4}}^{\infty}$ & $\sum_{i} \leq \sum \leq \sum \leq$ & 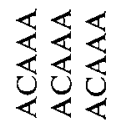 \\
\hline n & 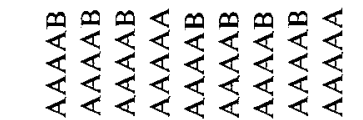 & $\sum_{i}^{\infty}$ & $\sum \sum \frac{1}{<} \leqslant$ & $\frac{\pi}{4}$ & 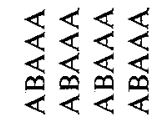 & 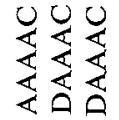 \\
\hline$\stackrel{\oiiint}{\varrho}$ & 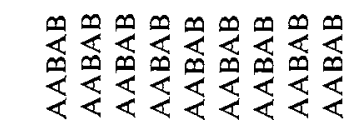 & 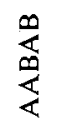 & $\begin{array}{l}\infty \\
\infty \\
\infty\end{array} \frac{\infty}{\infty}$ & $\frac{\infty}{\infty}$ & 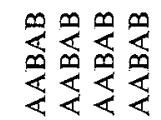 & 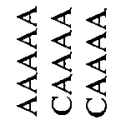 \\
\hline 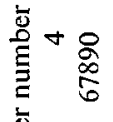 & 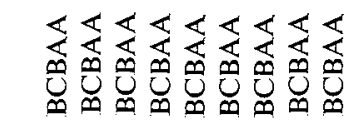 & 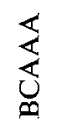 & $\sum_{\infty} \sum_{\infty}^{\infty} \bigcup_{\infty}$ & 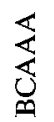 & 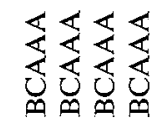 & 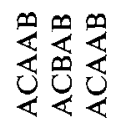 \\
\hline 篅 & 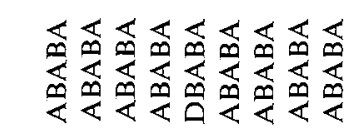 & $\sum_{\infty}^{\infty}$ & $\begin{array}{l}\infty \\
\sum\end{array} \frac{\infty}{\infty} \frac{\infty}{\infty}$ & 离 & 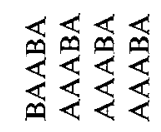 & $\sum_{i} \sum \frac{1}{\delta} \leqslant$ \\
\hline m & 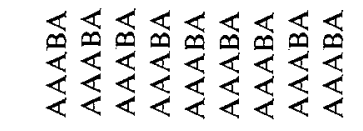 & $\underset{\infty}{\infty}$ & 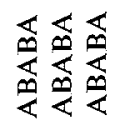 & 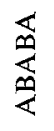 & 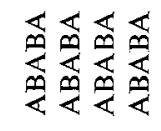 & 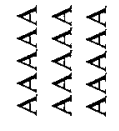 \\
\hline 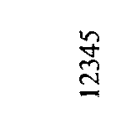 & 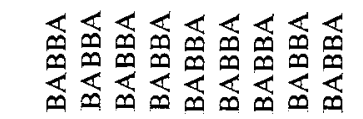 & $\underset{\infty}{\infty}$ & 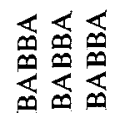 & 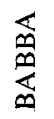 & 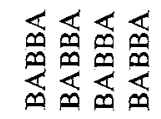 & $\sum \sum \sum \leqslant$ \\
\hline N & 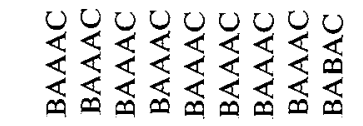 & $\sum_{\infty}^{\infty}$ & 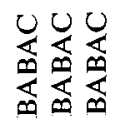 & 产 & 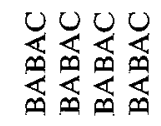 & 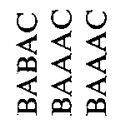 \\
\hline 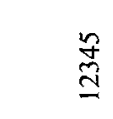 & 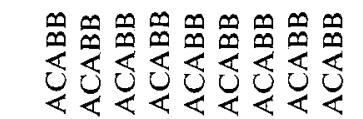 & 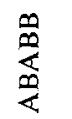 & 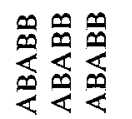 & 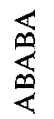 & 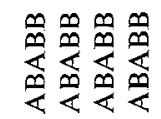 & $\begin{array}{l}\infty \\
\sum\end{array} \frac{\infty}{\infty} \frac{\infty}{<}$ \\
\hline - $\underset{\substack{\infty \\
6}}{\stackrel{8}{6}}$ & 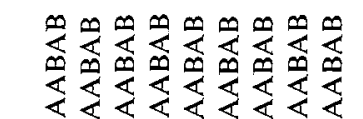 & $\sum_{\substack{\infty \\
<}}^{\infty}$ & 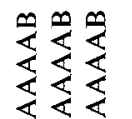 & $\sum_{i}^{\infty}$ & $\sum_{\infty}^{\infty} \sum_{\infty}^{\infty} \sum_{\infty}^{\infty} \sum_{\infty}^{\infty}$ & 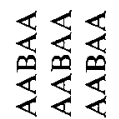 \\
\hline$\stackrel{n}{\stackrel{\Xi}{\Xi}}$ & 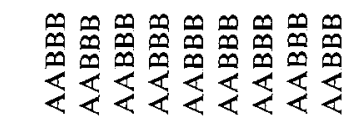 & 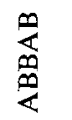 & 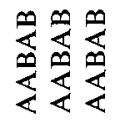 & 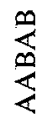 & 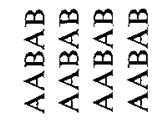 & 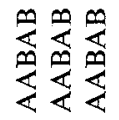 \\
\hline 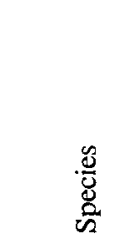 & 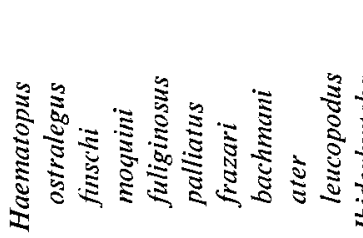 & $\bar{n}$ & $y$ & & 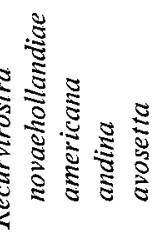 & 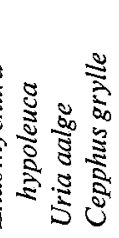 \\
\hline
\end{tabular}




\section{Skull characters}

Character 1: position of the lacrimal-ectethmoid complex (Figs 5, 6, 7)

The lower end of the lacrimal-ectethmoid complex is usually far from the jugal bar. In Pluvianus and the Burhinidae, however, it abuts the jugal bar. In the snipes and woodcocks the end of the complex is joined by a thin ribbon of bone to the postorbital and zygomatic processes, a condition which is clearly derived. Based on the distribution of states within

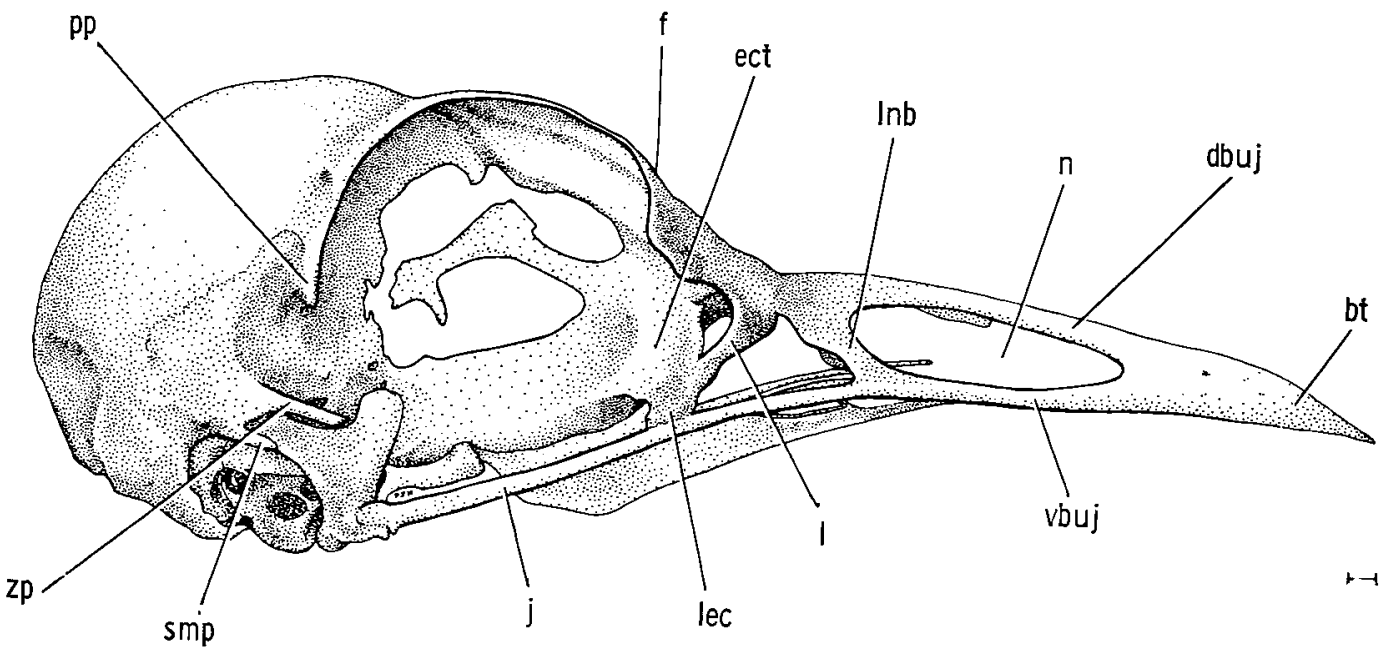

FIG. 5. Right lateral view of the skull of Pluvianus aegyptius (FM 93449). Abbreviations: bt, bill tip; dbuj, dorsal bar of the upper jaw; ect, ectethmoid; f, frontal; $j$, jugal bar; l, lacrimal; lec, lacrimal-ectethmoid complex; lnb, lateral nasal bar; n, nostril; pp, postorbital process; smp, suprameatic process; vbuj, ventral bar of the upper jaw; $\mathrm{zp}$, zygomatic process. Scale represents $1 \mathrm{~mm}$.

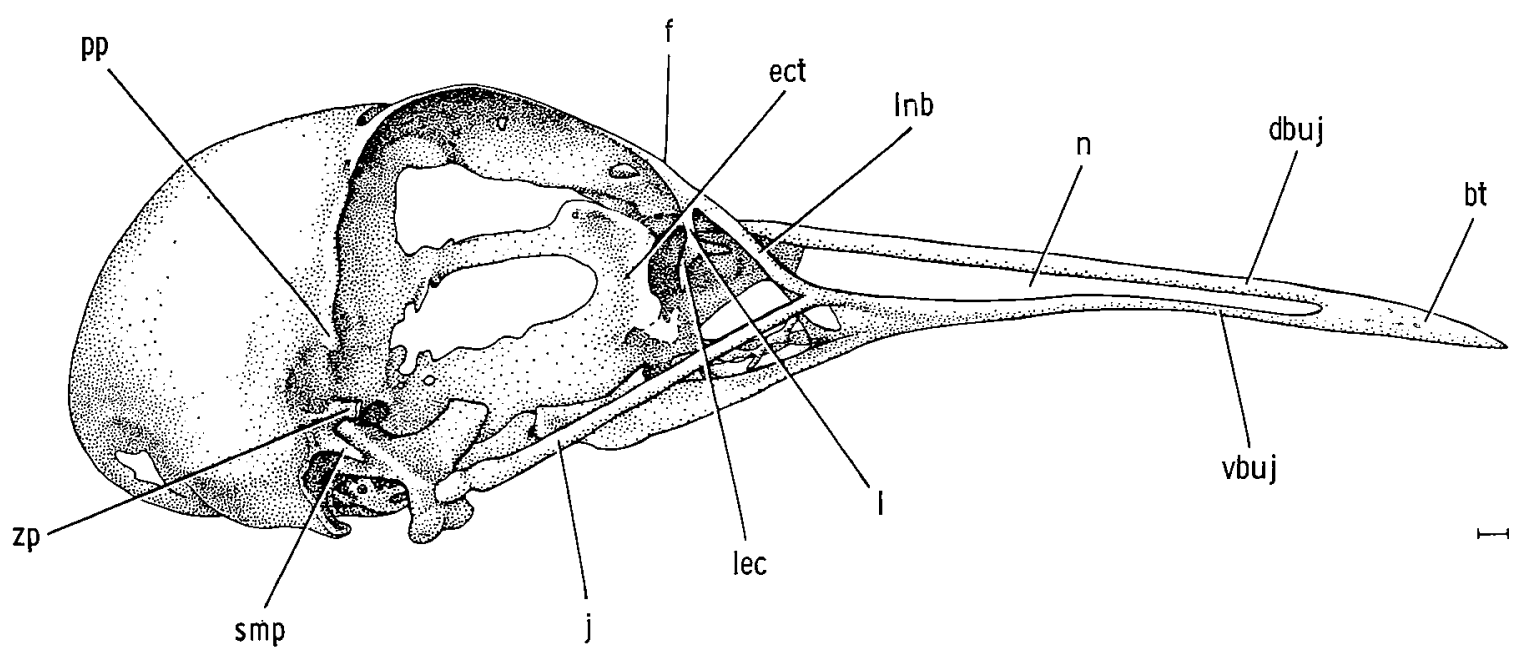

FIG. 6. Right lateral view of the skull of Thinornis novaeseelandiae (NZNM 1403). Abbreviations as in Fig. 5. Scale represents $1 \mathrm{~mm}$. 


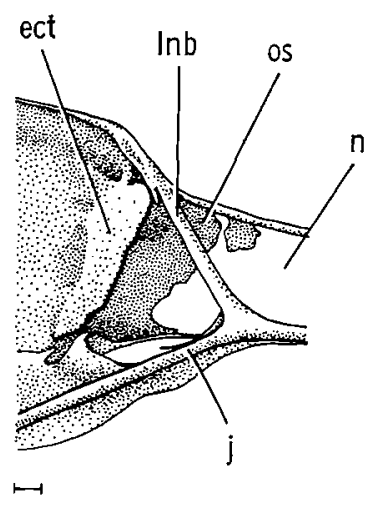

A

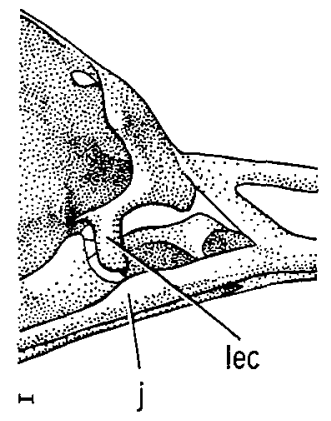

B

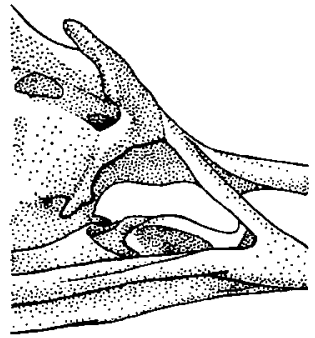

C

FIG. 7. Right lateral view of the skulls of three species. A. Attagis gayi (UMMZ 209968); B. Burhinus magnirostris (UMMZ 214183); C. Stercorarius pomarinus (UMMZ 153518). Abbreviations: ect, ectethmoid; j, jugal bar; lec, lacrimal-ectethmoid complex; Inb, lateral nasal bar; n, nostril; os, orbital septum. Scales represent $1 \mathrm{~mm}$.

the Charadriiformes, the condition in which the complex is far from the jugal bar is probably the primitive state.

Coding: primitive: complex not abutting jugal bar nor connected with postorbital process (A) (Figs 6, 7A, C); derived from primitive: complex abutting jugal bar (B) (Figs $5,7 \mathrm{~B}$ ); derived from primitive: complex connected with postorbital process (C) (not illustrated).

Character 2: fusion of lacrimal and ectethmoid (Figs 5, 6, 7)

In most Charadriformes and Columbiformes the lacrimals and ectethmoids are touching and usually fused. In a few of the Charadriiformes and in most of the Gruidae the two structures are not in contact, usually because the ectethmoids are reduced or absent. On the basis of the distribution of the states within the Charadriiformes, the fused or touching condition is probably the primitive state.

Coding: primitive: lacrimal and ectethmoid touching or fused (A) (Figs 5, 6, 7A, C); derived: lacrimal and ectethmoid not in contact (B) (Fig. 7B).

Character 3: fusion of the ectethmoid and frontals (Figs 5, 6, 7)

The most widely distributed condition for this character in the Gruiformes, Charadriiformes and Columbiformes is for there to be no connection or fusion between the ectethmoids and frontals; this is assumed to be the primitive state.

Coding: primitive: ectethmoid and frontal not in contact (B) (Figs 5, 6, 7); derived: ectethmoid and frontal in contact and often fused (A) (not illustrated).

\section{Character 4: angle between jugal bar and lateral nasal bar (Figs 5, 6, 7, 13)}

Lowe $(1915 b)$ used several angles between elements of the skull to separate his subfamilies Eroliinae and Tringinae of the Scolopacidae. One of these, the angle between the jugal bar and lateral nasal bar, or the angle between the jugal bar and the ventral bar of the upper jaw, as Lowe defined it, was used in this study. Two states were recognized, 
one in which the lateral nasal bar is relatively long, forming an angle of about $60^{\circ}$ or less with the jugal bar, and one in which the lateral nasal bar is relatively short, forming an angle of about $70^{\circ}$ or more with the jugal bar. Only the first state was found in the Gruiformes and Columbiformes and is probably the primitive state.

Coding: primitive: angle between lateral nasal bar and jugal bar about $60^{\circ}$ or less (A) (Figs 5, 7B, C, 13B, C); derived from primitive: angle between lateral nasal bar and jugal bar about $70^{\circ}$ or more (B) (Figs 6, 13A, D); derived from primitive: like (B), found in Thinocoridae, assumed to be a result of the short, finch-like bill and possibly of independent origin (C) (Fig. 7A).

Character 5: length of the zygomatic process relative to that of the suprameatic process (Fig. 5)

In most charadriiform birds the zygomatic process is longer than the suprameatic process; this condition is probably the primitive state. The condition in the snipes and woodcocks, in which this process is connected with the lacrimal-ectethmoid complex, is probably derived.

Coding: primitive: zygomatic process longer than suprameatic process (B) (Fig. 5); derived from primitive: zygomatic process shorter than suprameatic process (A) (not illustrated); derived from primitive: zygomatic process connected with lacrimal-ectethmoid complex (C) (not illustrated).

\section{Character 6: anterior development of the orbital septum (Figs 6, 7)}

In some Charadriiformes the orbital septum is poorly developed anterior to the region of the ectethmoid plate, while in others it extends far into the nostril and acts as a brace for the dorsal bar of the upper jaw. This condition in which the orbital septum extends anterior to the junction of the jugal bar and lateral nasal bar does not occur in the Gruiformes, Columbiformes, nor in most of the Charadriiformes and is thus probably a derived condition.

Coding: primitive: orbital septum not extending anterior to the junction of the jugal bar and lateral nasal bar (A) (Figs 6, 7A, B, C); derived: orbital septum extending anterior to junction of jugal bar and lateral nasal bar (B) (not illustrated).

Character 7: nostril type (Figs 5, 6,7)

It is probable from the distribution of nostril types among the Gruiformes, Charadriiformes, and Columbiformes that the schizorhinal nostril is the primitive condition for the Charadriiformes.

Coding: primitive: nostril schizorhinal (A) (Figs 6, 7A, C); derived: nostril holorhinal (B) (Figs 5, 7B).

\section{Character 8: form of the bill tip (Fig. 8)}

Lowe $(1915 b)$ used the morphology of the bill tip as a character to differentiate between his subfamilies Eroliinae and Tringinae of the Scolopacidae. I found his simple classification of two states inadequate to describe the diversity found throughout the Charadriiformes. In the Gruiformes, Columbiformes and most of the Charadriiformes the dorsal and ventral bars of the upper jaw are separate for most of the length of the bill, fusing for only a short distance at the tip. This generalized state is taken to represent the primitive 


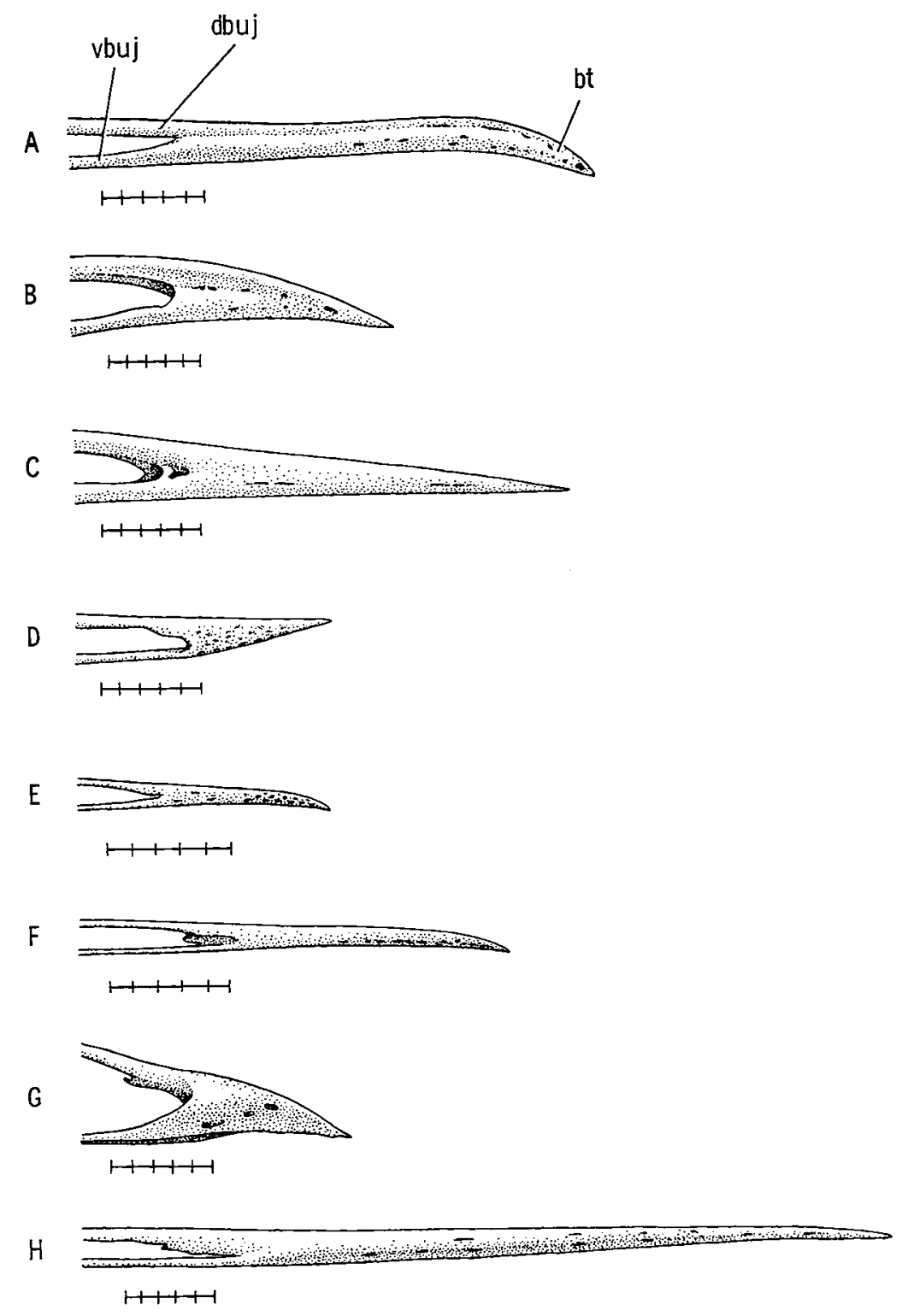

FIG. 8. Bills of eight species. A. Rostratula benghalensis (MVZ 120048); B. Larus philadelphia (UMMZ 153791); C. Gygis alba (UMMZ 210367); D. Arenaria interpres (UMMZ 216124); E. Actitis macularia (UMMZ 85037); F. Tringa glareola (UMMZ 219076); G. Attagis gayi (UMMZ 209968); H. Himantopus h. mexicanus (UMMZ 74110). Each scale division represents $1 \mathrm{~mm}$. abbreviations as in Fig. 5.

condition. At the beginning of this study the only derived state recognized was that in which the tip of the bill is relatively long, with the dorsal and ventral bars fused for about half the length of the bill. Within these two states there is still a considerable diversity of bill types. Later I recognized additional derived states for this character to describe 
better the diversity observed. I have assumed that all the derived types arose independently from the primitive state.

Coding: primitive: bill tip short, dorsal and ventral bars of upper jaw fused only near tip of bill (B) (Fig. 8B, E); derived from primitive: bill tip long, dorsal and ventral bars of upper jaw fused for about one-half length of bill (A) (Fig. 8C, F, H); derived from primitive: unique to Rostratula, generally like (A) but dorsal bar flattened with a distinctive ridge along top (C) (Fig. 8A); derived from primitive: short, stubby, finch-like tip, unique to Thinocoridae (D) (Fig. 8G); derived from primitive: generally like primitive state but tip a sharp, short cone, unique to Arenaria (E) (Fig. 8D); derived from primitive: like primitive state but with a distinct flange of bone on both sides of dorsal bar of upper jaw covering the nostril for about one-half length of bill, meeting but not fused to ventral bar of upper jaw; found only in phalaropes (F) (not illustrated).

Character 9: shape of the cross-section of the dorsal bar of the upper jaw (Fig. 9)

The dorsal bar of the upper jaw of most Charadriiformes (and of all Gruiformes and Columbiformes) is a simple, unreinforced bar; this condition is probably the primitive

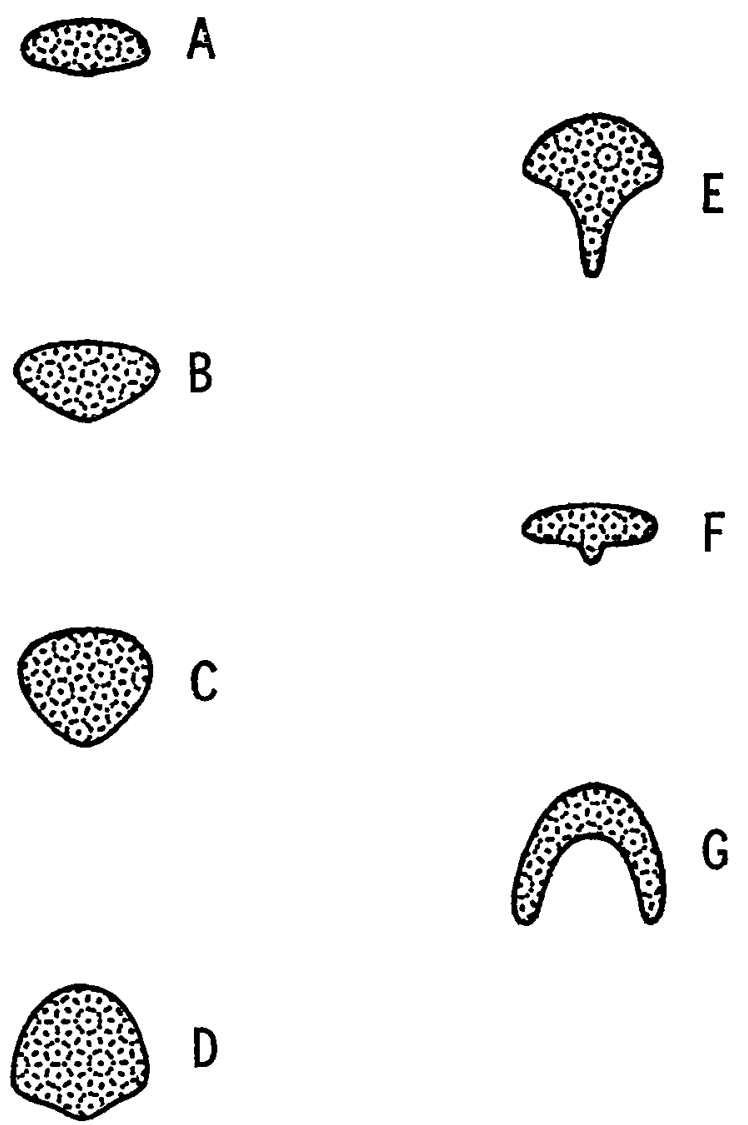

Fig. 9. Cross-sections of the dorsal bar of the upper jaw of representative charadriiform birds. A and B. extremes of the condition found in most species (see text); C. Gallinago; D. Scolopax; E. Limnodromus; F. Limicola; G. Limosa. 
state. Several species, especially members of the Scolopacidae, have bars which are distinctly reinforced. This reinforcement is correlated with a forward shift in the zone of bending in the rhynchokinetic skull of charadriiform birds (Schumacher, 1929; Burton, 1974), allowing the birds to open just the tip of the bill. This reinforcement appears to have evolved several times, since several distinctive morphologies exist for it. The typical stint (Calidris sp.) shows a slight thickening in the middle of the dorsal bar which in many species is hardly distinguishable from the primitive condition. I have coded all the species of Calidris, as well as the snipes and woodcocks, as belonging to the primitive condition regardless of the extent of the reinforcement. In many sandpipers and the curlews the cross-section of the bar is distinctly oval (Fig. 9B), in the snipes it is more or less teardropshaped (Fig. 9C), and in the woodcocks it is shaped like a haystack (Fig. 9D). These conditions were coded as primitive because other coding I considered would necessitate making a priori judgments about relatedness among the Scolopacidae. The derived states recognized are as follows: cross-section distinctly T-shaped (Fig. 9E), unique to Limnodromus (An alternative hypothesis would be that this shape evolved from or gave rise to the teardrop shape found in snipe.); cross-section broad and flat with a narrow reinforcing rod down the centre, shaped somewhat like a thumbtack (Fig. 9F), unique to Limicola (This condition may be related to the Limnodromus condition.); cross-section in the form of an inverted U (Fig. 9G), unique to Limosa. (Since completing this study I have found this condition present in some specimens of Xenus.)

Coding: primitive: cross-section of dorsal bar of upper jaw simple and unreinforced or, if reinforced, cross-section oval, teardrop- or haystack-shaped (A) (Fig. 9A, B, C, D); derived from primitive: cross-section T-shaped (B) (Fig. 9E); derived from primitive: cross-section like a thumbtack (C) (Fig. 9F); derived from primitive: cross-section like inverted U (D) (Fig. 9G).

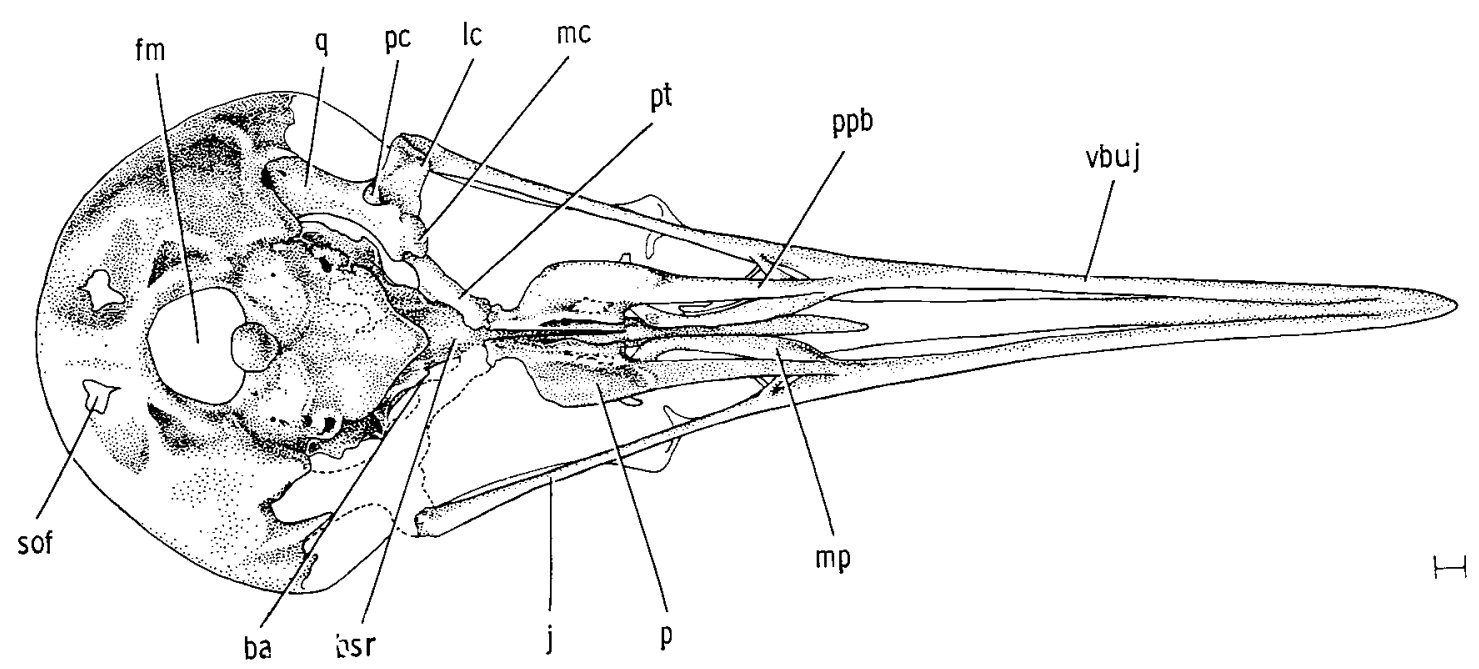

FIG. 10. Ventral view of the skull of Thinornis novaeseelandiae (NZNM 1403). Abbreviations: ba, basipterygoid articulation; bsr, basisphenoid rostrum; fm, foramen magnum; j, jugal bar; lc, lateral condyle of quadrate; mc, medial condyle of quadrate; mp, maxillopalatine; $p$, palatine; $p c$, posterior condyle of quadrate; $p p b$, prepalatine bar; pt, pterygoid; q, quadrate; sof, supraoccipital foramen; vbuj, ventral bar of upper jaw. Scale represents $1 \mathrm{~mm}$. 
Character 10: basipterygoid articulation of pterygoid and basipterygoid processes on basisphenoid rostrum (Fig. 10)

A well-developed basipterygoid process on the basisphenoid rostrum and a corresponding process located on the posterior edge of the pterygoid are present in many birds. In this study such processes were found in the skulls of adult Turnicidae, Rostratulidae, Jacanidae, Scolopacidae, Charadriidae, Pteroclidae, and Collimbidae; they were absent in the adult skulls of Podicipedidae, Gaviidae, Rallidae, Heliornithidae, Eurypygidae, Gruidae, Aramidae, Otididae, Thinocoridae, Chionididae, Glareolidae (except Peltohyas), Burhinidae, Dromadidae, Laroidea, and Alcae.

Jollie (1957) reports that many birds have basipterygoid processes early in development which are later enclosed by the parasphenoid. Such a process is illustrated in Uria by Parker (1890). Very young chicks of Chionis, Dromas, Rynchops, and some Laridae have such a process, though it is absent in the adults (Lowe, 1916a, b; Maillard, 1948; pers. obs.).

It is not clear from the distribution of the states in the outgroups or within the Charadriiformes which state is primitive. I have chosen the absence of the process in the adult as primitive because that state appears in most of the families examined in this study.

Coding: primitive: processes absent (A) (not illustrated); derived: processes present in adult (B) (Fig. 10).

\section{Character 11: medial condyle of quadrate (Fig. 10)}

Bock (1964) used the differences in the morphology of the three articular condyles of the quadrate as qualitative taxonomic characters to separate plovers and glareolids. In this study I use states of only the medial condyle. In the most widely distributed condition in the Charadriiformes there is a distinct groove on the anterolateral face of the condyle; this is probably the primitive state for the order. The groove is present in the Gruiformes but absent in the Columbiformes. While Bock found no groove in the Glareolidae, some specimens I have examined show a distinct dimple on the condyle. The position of the condyle in the Glareolidae, however, is very distinctive, with the condyle somewhat raised and twisted so that it faces outward.

Coding: primitive: distinct groove on anterolateral face of medial condyle of quadrate (A) (Fig. 10); derived from primitive: no groove on anterolateral face of medial condyle of quadrate (B) (not illustrated); derived from primitive: medial condyle of quadrate raised and twisted so that it faces outward; little or no grooving on anterolateral face (C) (not illustrated).

\section{Character 12: degree of fusion of the maxillopalatine-palatine complex (Figs 10, 11, 12)}

The degree of fusion of the maxillopalatines and the palatines varies considerably among the species in the three orders examined. The most widespread and thus most likely primitive condition within the Charadriiformes is for the maxillopalatines to be delicate structures having little or no fusion with the palatines. I have recognized two derived states which appear to have developed sequentially from the primitive state, a state in which there is considerable but not complete fusion between the maxillopalatines and palatines and a state in which the two structures are completely fused, often with complete loss of an identifiable maxillopalatine. 


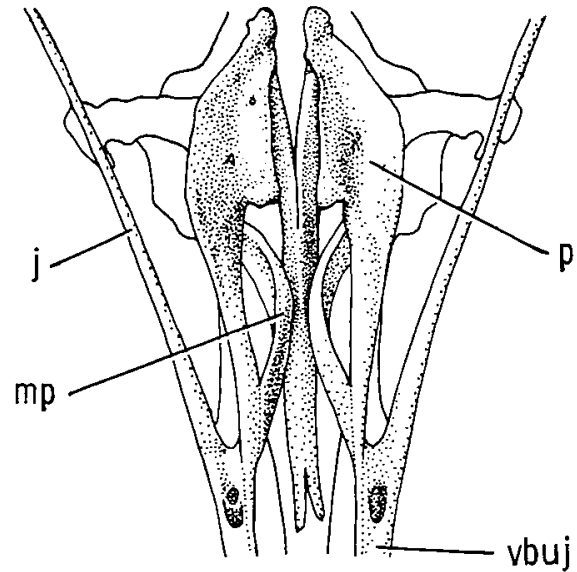

A

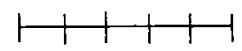

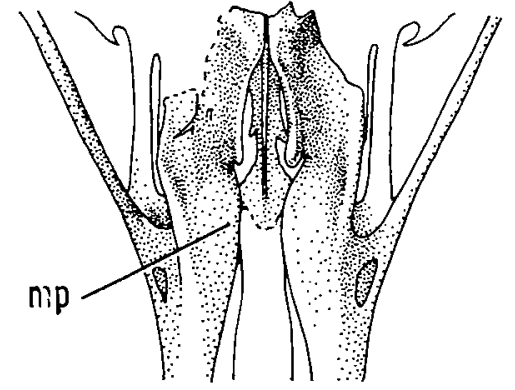

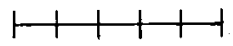

B

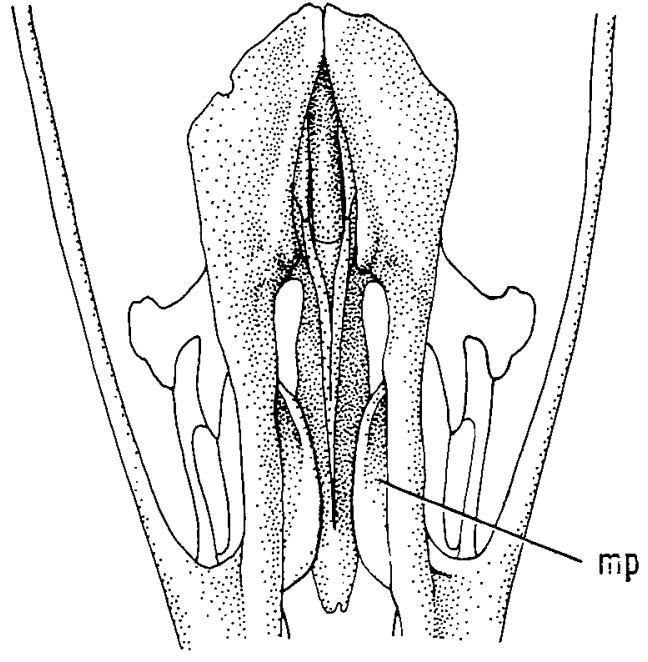

C

Fig. 11. Ventral view of the palates of four species. A. Nycticryphes semicollaris (UMMZ 157022); B. Coenocorypha aucklandii (NZNM 1406); C. Cepphus grylle (UMMZ 151022); D. Scolopax rusticola (UMMZ 156165). Abbreviations as in Fig. 10. Each scale unit represents $1 \mathrm{~mm}$.

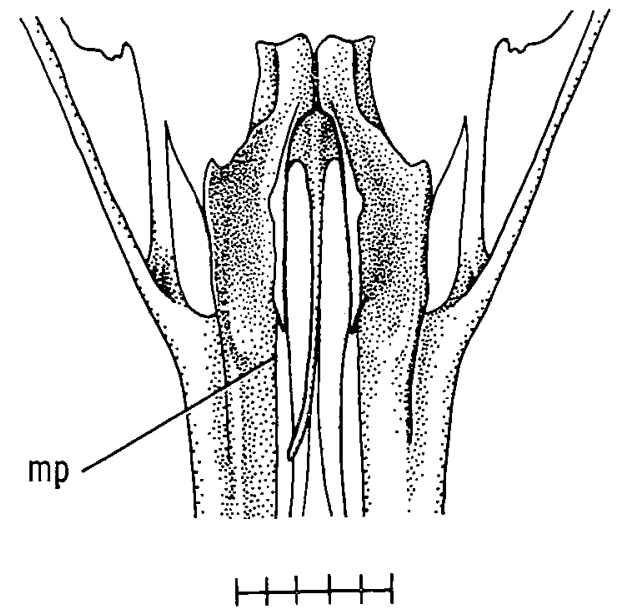

D 


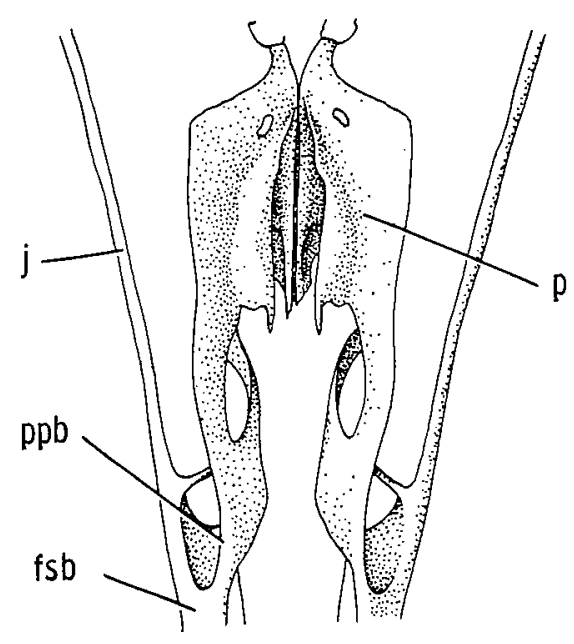

A
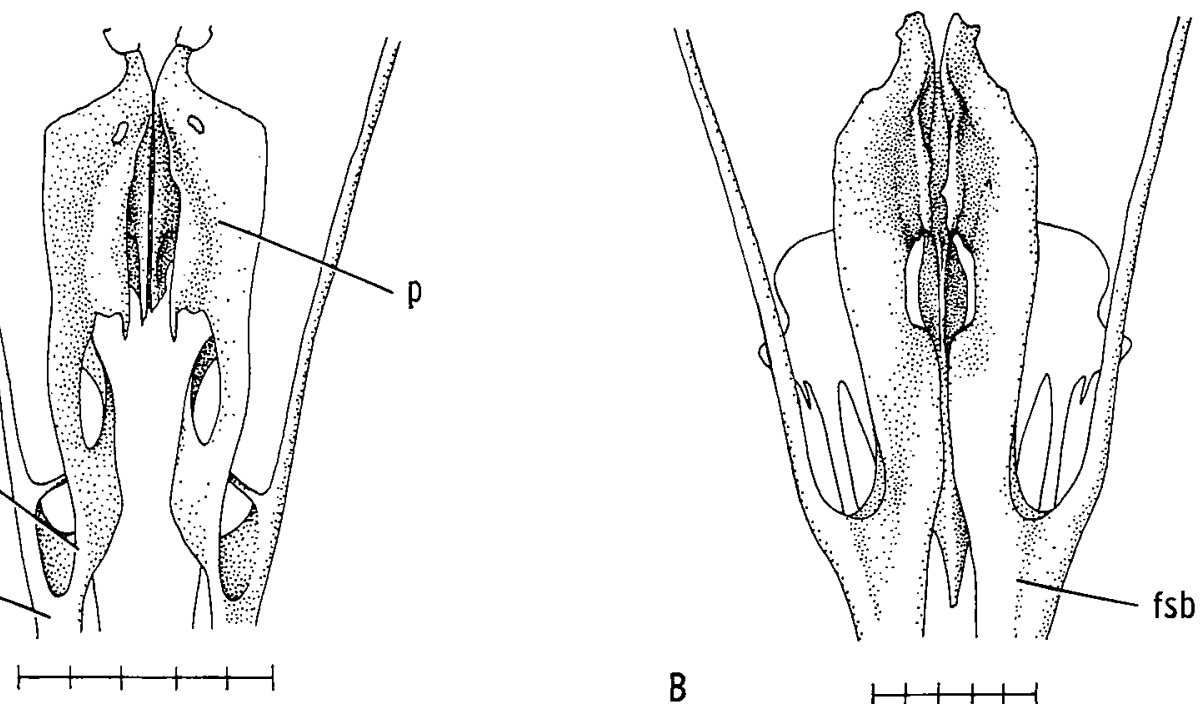

B
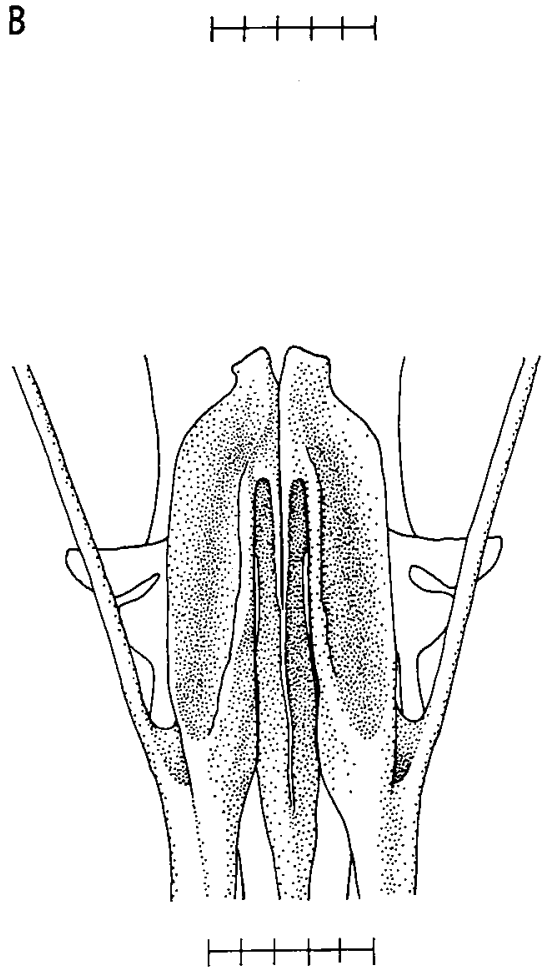

D

C

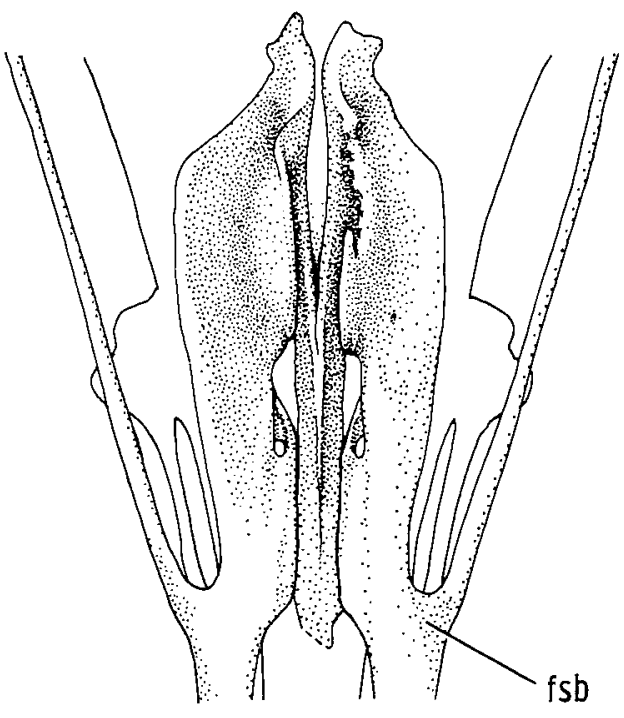

FIG. 12. Ventral view of the palates of four species. A. Prosobonia cancellatus (AMNH 6592); B. Bartramia longicauda (UMMZ 151204); C. Numenius minutus (USNM 347648); D. Limosa haemastica (UMMZ 154491). Abbreviations: fsb, flat sheet of bone; $j$, jugal bar; p, palatine; ppb, prepalatine bar. Each scale unit represents $1 \mathrm{~mm}$. 
Coding: primitive: maxillopalatines and palatines with little or no fusion (A) (Figs 10, $11 \mathrm{~A}, \mathrm{C}, 12 \mathrm{~A}$ ); derived from primitive: maxillopalatines and palatines considerably but not completely fused (B) (Fig. 12B, C, D); derived from (B): two structures completely fused (C) (Fig. 11B, D).

Character 13: configuration of the junction of the jugal bar, prepalatine bar, and ventral bar of the upper jaw (Figs 10, 11, 12)

A groove on the ventral surface of the ventral bar of the upper jaw extends anteriorly from the area where the jugal bar, prepalatine bar, and ventral bar of the upper jaw meet in all Charadriiformes except Numenius, Bartramia, and Prosobonia. In the latter three a flat sheet of bone extends across this region. This sheet of bone is considerably reduced in Prosobonia.

Coding: primitive: no sheet of bone over area where jugal bar, prepalatine bar, and ventral bar of upper jaw meet (A) (Figs 10,11A, B, C, D, 12D); derived: a sheet of bone over this area (B) (Fig. 12A, B, C).
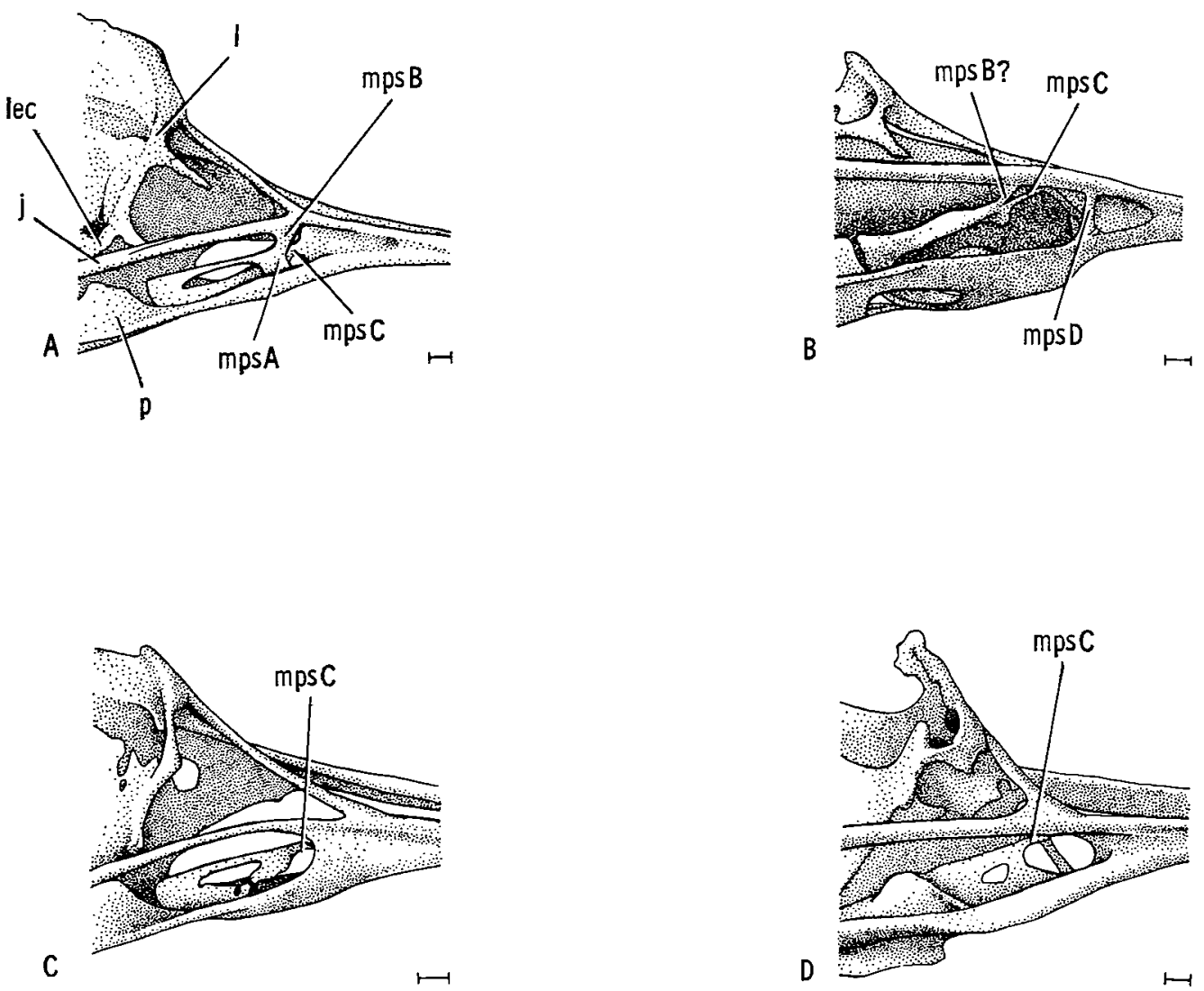

FIG. 13. Ventrolateral view of the skulls of four species. A. Charadrius montanus (UMMZ 220120); B. Heteroscelus incanus (UMMZ 151698); C. Numenius minutus (USNM 347648); D. Aphriza virgata (UMMZ 215473). Abbreviations: j, jugal bar; I, lacrimal; lec, lacrimal-ectethmoid complex; mpsA, maxillopalatine strut A; mpsB, maxillopalatine strut $B$; mpsC, maxillopalatine strut $C ;$ mpsD, maxillopalatine strut $D ; p$, palatine. Scales represent $1 \mathrm{~mm}$. 
Characters 14, 15, 16, 17: maxillopalatine struts (Fig. 13)

The complex architecture of the maxillopalatine in the Charadriiformes is characterised by the presence or absence of at least four different bony struts. Lowe (1931a) and Zusi \& Jehl (1970), whose designations A, B, C and D are followed here, used these struts as qualitative taxonomic characters to separate the Scolopacidae and Charadriidae but did not speculate on which conditions might be primitive or derived. In many but not all of the Gruiformes and Columbiformes the maxillopalatine is hollow and egg-shaped; it is similar in some Glareolidae and Laridae but is usually perforated in several places. The primitive condition in the Charadriiformes is thus probably that of an almost entire maxillopalatine with the degree of ossification decreasing during evolution of the group. The presence of struts $\mathrm{A}$ and $\mathrm{C}$ is therefore primitive, representing the remnants of a more complete ossification of the maxillopalatine.

Some specimens of Charadrius cucullatus and those of species of the Haematopodidae, Ibidorhynchidae, and Recurvirostridae appear to lack strut A. Other specimens, however, have a distinct raised arch of bone on the lateral-facing medial wall of the maxillopalatine. This arch probably represents strut $\mathrm{A}$ in those species where the maxillopalatines have become compressed during development such that the lateral and medial surfaces of the maxillopalatines have fused.

Strut B, which connects the maxillopalatine with the jugal bar, is present in the Gruiformes and Columbiformes; on the basis of this distribution, the presence of strut B is probably the primitive condition. As noted by Zusi \& Jehl (1970), some specimens of Tringa have a strut similar to B while other specimens of the same species lack it. It is impossible to determine from adult skulls whether B and C are both present in Tringa but lie so close together that they sometimes fuse and cannot be distinguished, or whether $\mathrm{B}$ is absent in Tringa and $\mathrm{C}$ sometimes sends a branch out to the jugal bar. All species of Tringa were coded as having strut $\mathrm{B}$. While it is unclear whether strut $\mathrm{D}$, which is unique to the Tringinae, is actually a maxillopalatine structure, its presence, however, is clearly a derived condition.

Coding: Character 14 (strut A): primitive: present, either as a distinct strut or as an ossified ridge on lateral surface of maxillopalatine (B) (Fig. 13A); derived: absent (A) (Fig. 13B, C, D);

Character 15 (strut B): primitive: present (B) (Fig. 13A, B); derived: absent (A) (Fig. $13 \mathrm{C}, \mathrm{D})$;

Character 16 (strut C): primitive: present (B) (Fig. 13A, B, C, D); derived: absent: (A) (not illustrated);

Character 17 (strut D): primitive: absent (A) (Fig. 13A, C, D); derived: present (B) (Fig. 13B).

\section{Character 18: supraoccipital foramina (Figs 10, 14)}

A pair of supraoccipital foramina just posterior to the foramen magnum is present in some birds. In this study the condition was found in the Gruidae, Rostratulidae, Scolopacidae, Charadriidae, Glareolidae, and Alcae, though not necessarily in every species. On the basis of the distribution of the states within the Charadriiformes, absence of such foramina is probably the primitive condition.

Coding: primitive: supraoccipital foramina absent (A) (not illustrated); derived: a pair of supraoccipital foramina present (B) (Figs 10, 14). 
Character 19: shape of the foramen magnum (Figs 10,14)

The shape of the foramen magnum for most of the Charadriiformes is essentially the same. In some species of Scolopacidae, however, it is distinctly heart-shaped.

Coding: primitive: foramen magnum not distinctly heart-shaped (A) (Figs 10, 14B); derived: foramen magnum distinctly heart-shaped (B) (Fig. 14A).

Character 20: position of the nasal glands (Fig. 15)

All birds possess distinctive head glands known as nasal glands (Shoemaker, 1972). In marine birds particularly these glands have been demonstrated to excrete salt (SchmidtNielsen, 1960) and thus are often called salt glands. Staaland (1967) has shown a strong correlation among ecology, nasal gland anatomy, and function for several species of

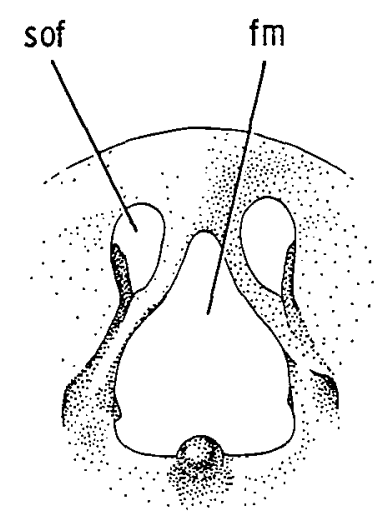

A

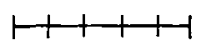

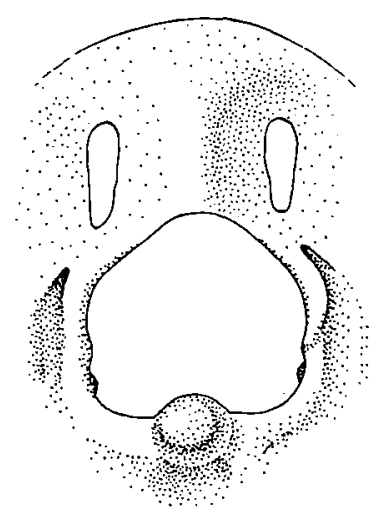

B

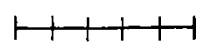

FIG. 14. Occipital region of the skulls of two species. A. Calidris melanotos (UMMZ 70485); B. Arenaria interpres (UMMZ 216124). Abbreviations: fm, foramen magnum; sof, supraoccipital foramen. Each scale unit represents $1 \mathrm{~mm}$.

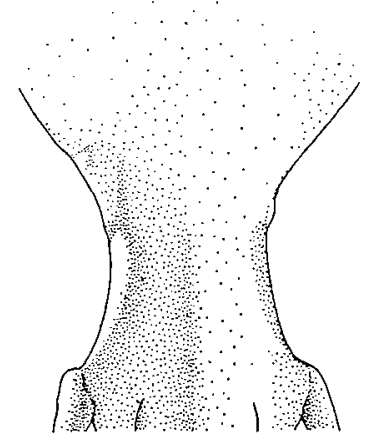

A

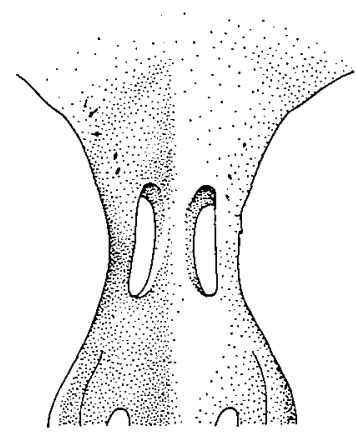

B

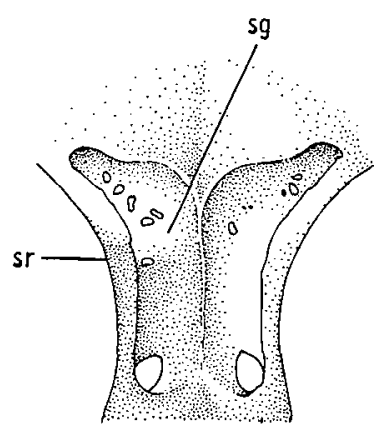

C

FIG. 15. Dorsal view of the frontal region of the skulls of three species. A. Ibidorhyncha struthersii (USNM 292767); B. Cursorius cursor (UM 2964); C. Thinornis novaeseelandiae (NZNM 1403). Abbreviations: sg, supraorbital groove; sr, supraorbital rim. Each scale unit represents $1 \mathrm{~mm}$. 
charadriiform birds. In these birds the glands usually lie in supraorbital grooves in the skull; in some species with well-developed glands these grooves are so large that the supraorbital rims are absent. Lowe (1922) used the extent of ossification of the supraorbital rims of plover skulls as a generic character. Bock (1958) showed that the correlation between skull ossification and plumage colour which Lowe used is better explained as adaptations to similar environments rather than as indicators of phylogenetic relationship. Yudin (1965) believed that "well-developed nasal glands are a primordial feature of the family Charadriidae, which to some extent are reduced independently and at different times in the various members of this group under the influences of changes in mode of life ... all Charadriidae with weakly developed nasal glands are specialized forms". Apparently, Yudin, like Lowe, equated phylogenetic development of the glands with the relative size of the glands found in the supraorbital position, since he was uncertain about the significance of the apparent interorbital position of the gland in Pluvianus.

Studying the ontogeny of the gland might be a better method of elucidating phylogenetic relationship. This has been described for a few birds by Marples (1932) and Technau (1936). Technau classified birds on the basis of the location of the nasal gland; his schematic representation (Technau, 1936, Fig. 23) could be taken as a possible evolutionary series. The positions described by Technau are preorbital, preorbital-interorbital, interorbital, interorbital-exorbital, exorbital, exorbital-supraorbital and supraorbital. Correct identification of these states, however, requires fresh or well-preserved intact specimens, which were unavailable for most of the species I studied. I was able to distinguish at least three different states from skulls: supraorbital, in which a distinct groove is present; exorbital, in which the supraorbital rim is missing even though there is no supraorbital groove (This state was verified in a preserved specimen of Jacana, in which a small nasal gland is present, replacing the supraorbital rim.); and neither of the above, in which case the gland was assumed to be either inter- or preorbital. There is no indication of the position of the nasal gland on the skull of Scolopax, nor could I locate a gland in three poorly-preserved heads of Philohela minor. Technau lists S. rusticola as having a preorbital gland. The three skulls of Pluvianus I examined show a small, shallow, supraorbital nasal gland groove. The distribution of the states of the gland among the Gruiformes and Columbiformes was not helpful in determining the primitive state of this character; the distribution of states among the Charadriiformes indicates that the primitive condition is supraorbital.

Coding: primitive: supraorbital nasal gland (C) (Fig. 15C); derived from primitive: exorbital nasal gland (B) (not illustrated); derived from (B): nasal gland neither supraorbital nor exorbital (A) (Fig. 15A); derived from primitive: like primitive state but groove for gland has become a foramen in top of skull; found only in some species of Cursorius (D) (Fig. 15B).

\section{Mandible characters}

\section{Character 21: prearticular process of mandible (Fig. 16)}

The prearticular process of the mandible in Charadriiformes is variable. In the most widely distributed and probably primitive state there is a flange on the upper side of the distal end of the process. In the snipes and woodcocks the process is expanded and hollowed, and the flange is absent; in the Glareolidae the flange is absent; and in the 
A

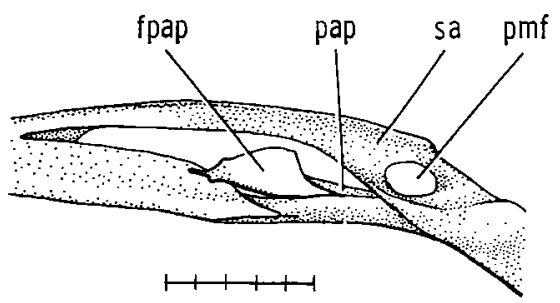

C

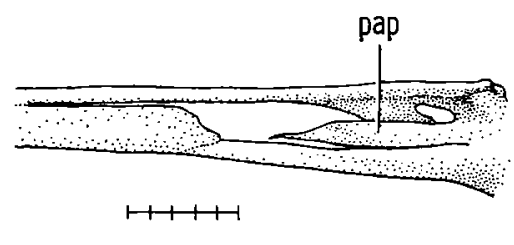

$\mathrm{E}$

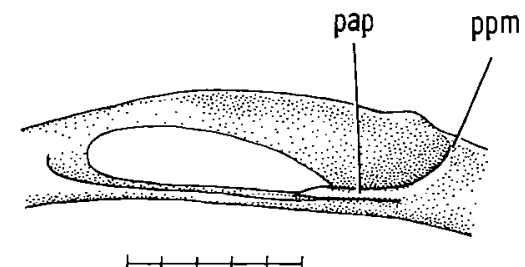

B

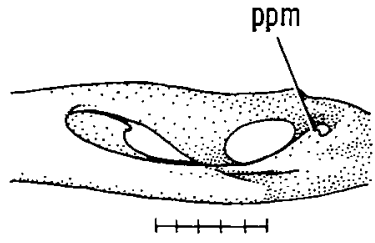

(

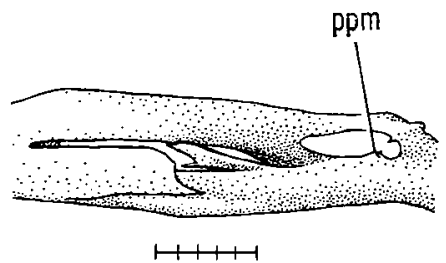

$F$

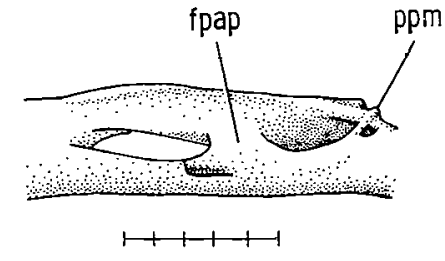

FIG. 16. Medial view of the mandibles of six species. A. Nycticryphes semicollaris (UMMZ 157021); B. Metopidius indicus (UMMZ 214551); C. Coenocorypha aucklandii (NZNM 1406); D. Catoptrophorus semipalmatus (UMMZ 156426); E. Pluvianellus socialis (SDSNH 37901); F. Thinornis novaeseelandiae (NZNM 1403). Abbreviations: fpap, flange of prearticular process; pap, prearticular process; pmf, posterior mandibular fossa; ppm, posterior process of mandible; sa, supraangular. Each scale unit represents $1 \mathrm{~mm}$.

Charadriidae the flange extends to and is usually fused with the supraangular except for Anarhynchus frontalis, in which the flange is absent.

Coding: primitive: flange on prearticular process but not fused to supraangular (A) (Fig. 16A, B, D); derived from primitive: flange on prearticular process fused to supraangular (B) (Fig. 16F); derived from (B): no flange on prearticular process; unique to $A$. frontalis (D) (not illustrated); derived from primitive: no flange on prearticular process (C) (Fig. 16E); derived from primitive: prearticular process expanded and hollow, no flange (E) (Fig. 16C).

Character 22: posterior process of mandible (Fig. 16)

At the attachment of aponeurosis XI of M. pseudotemporalis superficialis pt. 1 to the mandible is located the posterior process of the mandible (point $R$ of Bams, 1956). In most charadriiform birds the process arises on the prearticular; this is assumed to be the 
A
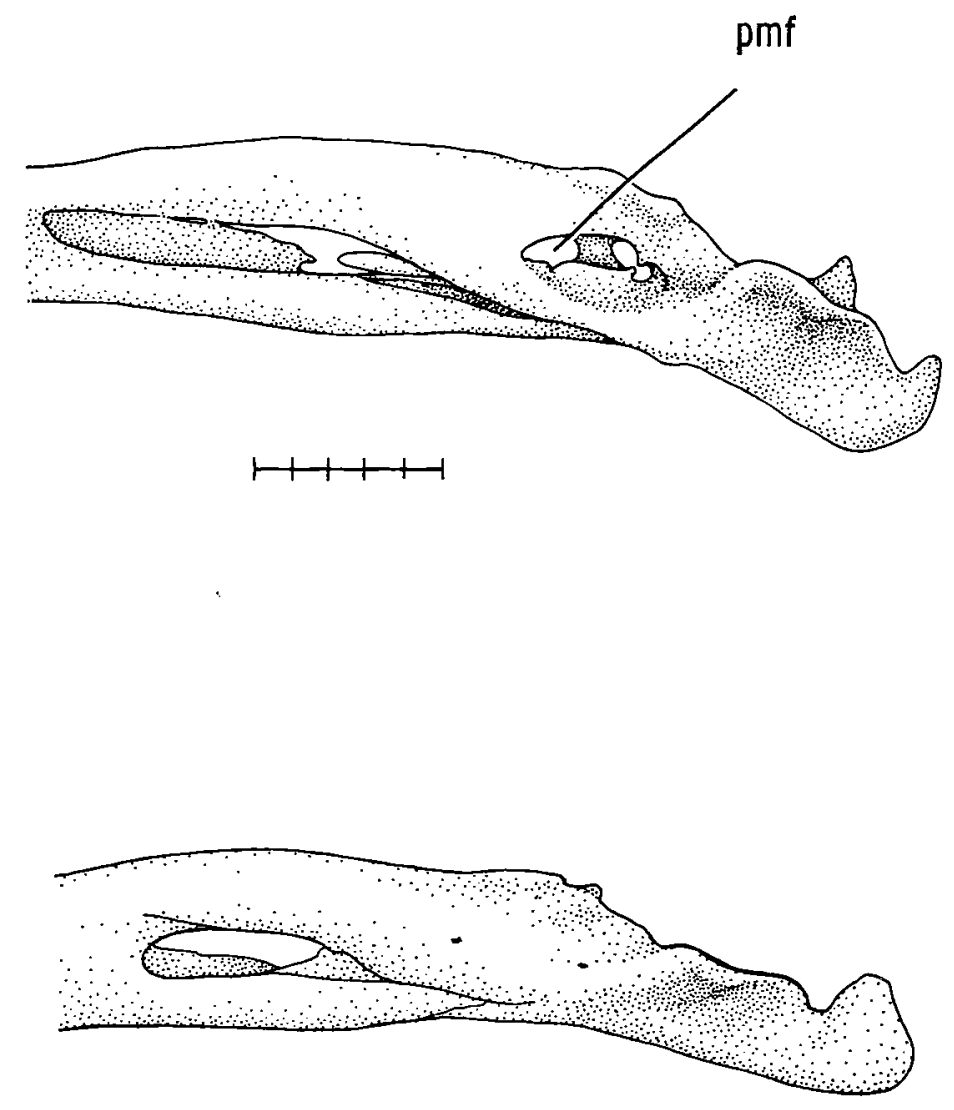

B
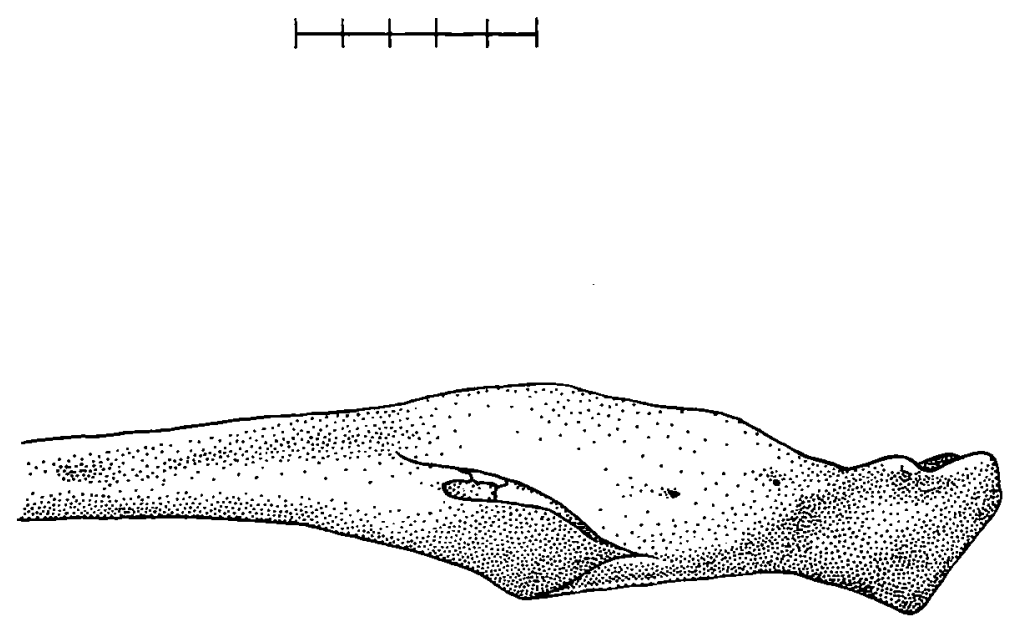

C

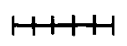

FIG. 17. Lateral view of mandibles of three species. A. Aphriza virgata (UMMZ 219182); B. Thinornis novaeseelandiae (NZNM 1403); C. Stercorarius pomarinus (UMMZ 153518). Abbreviation: pmf, posterior mandibular fossa. Each scale unit represents $1 \mathrm{~mm}$. 
primitive state. In some of the Scolopacidae the process is found on the supraangular, but there is a corresponding bump on the prearticular. In the snipes and woodcocks the position of the process is shifted posteriad on the mandible.

Coding: primitive: posterior process on prearticular in normal position (A) (Fig. 16A, $\mathrm{B}, \mathrm{E}, \mathrm{F})$; derived from primitive: process normal with corresponding bump on supraangular (B) (Fig. 16D); derived from primitive: process shifted posteriad on mandible (C) (Fig. 16C).

Character 23: posterior mandibular fossa (Fig. 17)

Most of the Gruiformes and Charadriiformes examined have a posterior fossa in the mandible; it is absent in the Columbiformes.

Coding: primitive: posterior mandibular fossa present (A) (Fig. 17A); derived: posterior mandibular fossa absent (B) (Fig. 17B, C).

\section{Head and neck muscle characters}

Characters 24, 25, 26, 27, 28, 29 and 30 (not illustrated)

These characters are based on tabulations of the positions of attachment on the neck vertebrae of three neck muscles which insert on the cranium, as published by Burton (1971, 1972, 1974). Since I had no information on the states found in the Alcae and information on only four species of the Laridae (Zusi, 1962), the alcids examined were coded as if they had the primitive state for these characters, and the characters were ignored in the analysis of the Laroidea. I also had to estimate the states for some species for which I had skeletons but which Burton (1974) did not examine. The state of a presumed closest relative was used. Usually all the species in a genus had the same state.

Coding: Character 24: presence of an origin of M. complexus on vertebra 3: primitive: present (A); derived: absent (B);

Character 25: presence of an origin of $\mathrm{M}$. complexus on vertebra 4: primitive: present (A); derived: absent (B);

Character 26: presence of an origin of $M$. complexus on vertebra 5: primitive: present (A); derived: absent (B);

Character 27: presence of an origin of $\mathrm{M}$. complexus on vertebra 6: primitive: present (A); derived: absent (B);

Character 28: presence of an origin of $\mathrm{M}$. complexus on vertebra 7: primitive: present (A); derived: absent (B);

Character 29: sites of origin of M. splenius capitis: primitive: origin only on vertebra 2 (A); derived: origin on vertebrae 2 and 3 (B);

Character 30: sites of origin of $M$. rectus capitis: primitive: origins of M. rectus capitis on vertebrae 1-5 (A); derived from primitive: origins on vertebrae 1-4 only (B); derived from primitive: origins on vertebrae $1-3$ only (C).

\section{Axial skeleton characters}

Character 31: number of caudal vertebrae, omitting the pygostyle (not illustrated)

The number of caudal vertebrae in the Charadriiformes varies from five to nine. The most widely distributed state in the order is eight; this is probably the primitive state. It is 
assumed that the evolution of this character has proceeded with a loss or gain of only one vertebra at a time. All Jacanidae have five vertebrae except Hydrophasianus chirurgus, which has six. The sixth caudal vertebra in chirurgus is assumed to be derived, along with the greatly elongated tail of the species, from the normal five of the Jacanidae.

Coding: primitive: eight caudal vertebrae (B); derived in single steps of vertebrae, increasing or decreasing from eight: 9 (C), 7 (A), 6 (D), 5 (E), and 6 in Jacanidae (G).

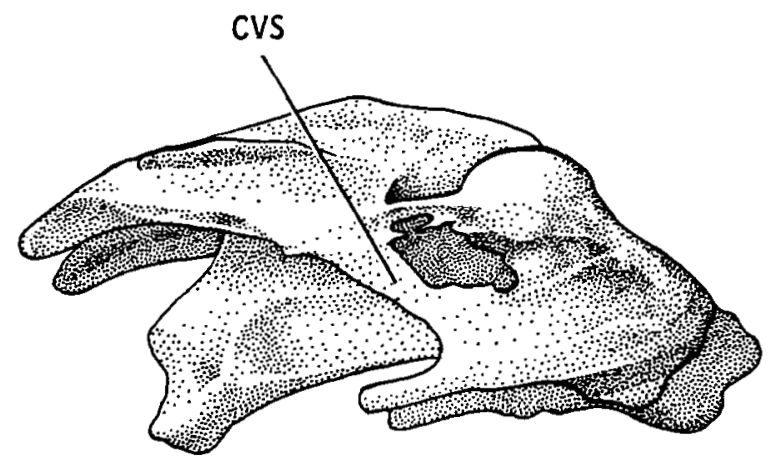

A
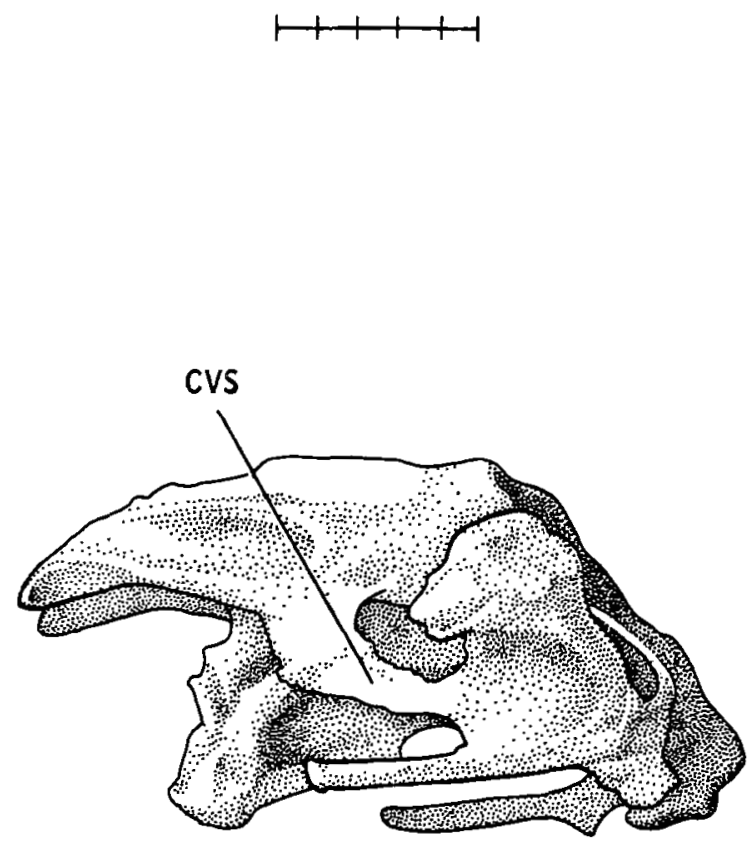

B

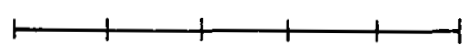

FIG. 18. Eighth cervical vertebra of two species. A. Vanellus melanopterus (UMMZ 220264); B. Burhinus magnirostris (UMMZ 214183). Abbreviation: cvs, cervical vertebral strut. Each scale unit represents $1 \mathrm{~mm}$. 
Character 32: number of neck vertebrae, omitting those with unfused cervical ribs (not illustrated).

The most common and probably primitive state in Charadriiformes is 13 neck vertebrae.

Coding: primitive: 13 neck vertebrae (A); derived from primitive: 12 neck vertebrae (B); derived from primitive: 14 neck vertebrae (C).

\section{Character 33: cervical vertebral strut (Fig. 18)}

The presence of a distinctive strut on the cervical vertebrae of vanelline plovers was first described by Zusi \& Jehl (1970). A similar strut is on the cervical vertebrae of the Burhinidae and on a few vertebrae of one of three skeletons of Pluvianus which I examined. No such strut is found in the Gruiformes or Columbiformes; its presence in the Charadriiformes is probably a derived state. I originally coded the presence of a strut as the same state for the lapwings and the thick-knees, but after an initial analysis of the cladistic relationships within the Charadrii indicated that there is no close relationship between these groups I recoded the strut as two different states.

Coding: primitive: no cervical vertebral strut (A) (not illustrated); derived from primitive: cervical vertebral strut in Vanellinae (B) (Fig. 18A); derived from primitive: cervical vertebral strut in Burhinidae (C) (Fig. 18B).

\section{Character 34: type of dorsal vertebrae (not illustrated)}

Two types of dorsal vertebrae are found in the Charadrifformes, heterocoelous (articular surfaces saddle-shaped) and opisthocoelous (anterior articular surface convex and posterior articular surface concave). The heterocoelous condition is the most widespread in all birds and is the only condition found in the Gruiformes and Columbiformes. It is thus probably the primitive condition in the Charadriiformes.

Coding: primitive: dorsal vertebrae heterocoelous (A); derived: dorsal vertebrae opisthocoelous (B).

\section{Pectoral girdle characters}

Character 35: number of sternal costal processes (Figs 19, 20)

The number of sternal costal processes is variable within the Gruiformes, Charadriiformes and Columbiformes, with five and six being the most widely distributed states. Within the Charadriiformes the six-process condition is the most widely distributed and probably primitive state.

Coding: primitive: six costal processes on sternum (A) (Fig. 21); derived from primitive: five costal processes (B) (Fig. 22); derived from (B): four costal processes (C) (not illustrated); derived from primitive: seven costal processes (D) (not illustrated).

\section{Character 36: medial sternal notch (Figs 19, 20)}

The sternum of all Charadriiformes is notched, all species having a lateral notch and some species having both a lateral and a medial notch. Except in the Otididae the medial notch is absent in the Gruiformes; it is present in the Columbiformes. Based on its distribution within the order, the absence of a medial notch is probably primitive in the Charadriiformes. This character, however, is not stable within the Scolopacidae, both states occurring in a series of specimens of some species. In addition, some specimens were 


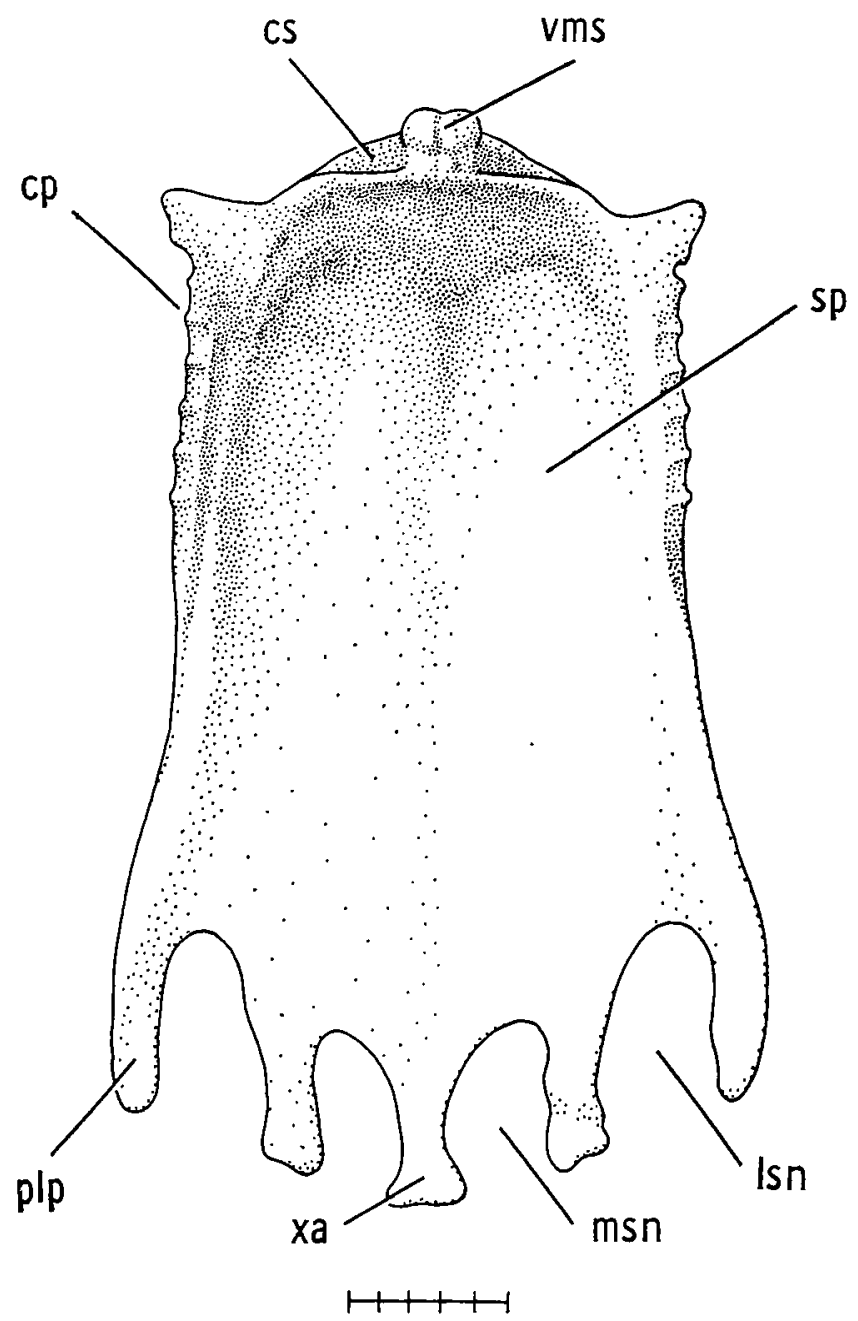

FIG. 19. Dorsal view of the sternum of Thinornis novaeseelandiae (NZNM 1403). Abbreviations: $\mathrm{cp}$, costal process; cs, coracoidal sulcus; lsn, lateral sternal notch; msn, medial sternal notch; plp, posterior lateral process; sp, sternal plate; vms, ventral manubrial process; xa, xiphial area. Each scale unit represents $1 \mathrm{~mm}$.

intermediate, with a medial notch on only one side or with medial notches present only as foramina. Any species in which at least some of the specimens had a medial notch was coded as having the notch.

Coding: primitive: medial sternal notch absent (A) (Fig. 20); derived: medial sternal notch present (B) (Fig. 19).

Character 37: relative lengths of the posterior lateral sternal process and xiphial area (Figs 19, 20)

The posterior lateral sternal process does not extend posteriad as far as the xiphial area in most Charadriiformes; this is probably the primitive state. 


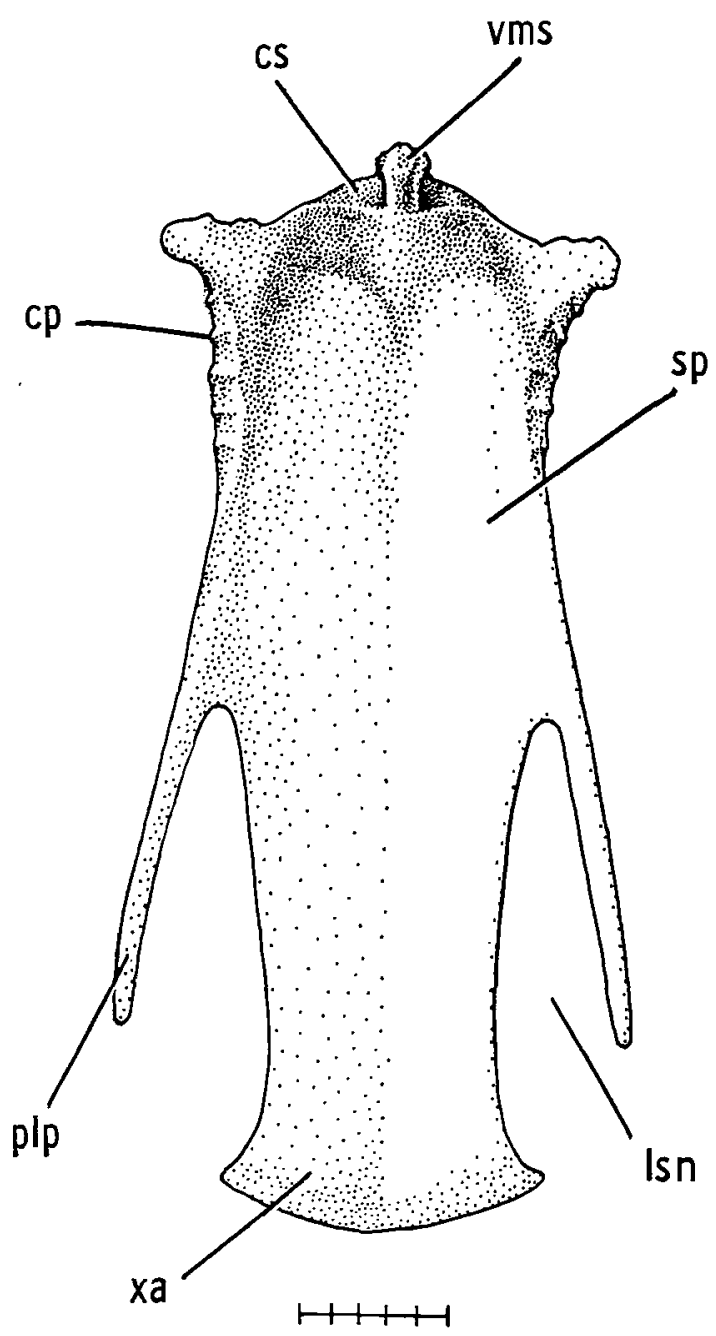

FIG. 20. Dorsal view of the sternum of Nycticryphes semicollaris (UMMZ 157021). Abbreviations as in Fig. 19. Each scale unit represents $1 \mathrm{~mm}$.

Coding: primitive: posterior lateral sternal process not extending as far posteriad as the xiphial area (C) (Figs 19, 20); derived from primitive: posterior lateral sternal process and xiphial area extending about equal distance posteriad (A) (not illustrated); derived from (A): posterior lateral sternal process extending farther posteriad than xiphial area (B) (not illustrated) (No charadriiform had this state.)

Character 38: ventral manubrial spine of sternum (Figs 19, 20)

A well-developed ventral manubrial spine is characteristic of the Charadriiformes but is generally absent in the Gruiformes and Columbiformes. Usually in the Charadriiformes a distinct ridge from the spine to the sternal plate forms a wall between the medial ends of the coracoidal sulci. This is probably the primitive state. 
Coding: primitive: distinct ridge from ventral manubrial spine to sternal plate forming a wall between medial ends of coracoidal sulci (A) (Figs 19, 20); derived: ridge absent (B) (not illustrated).

Character 39: coracoidal foramen (Fig. 21)

A distinct foramen is present in the coracoid of many birds. Its distribution among the Gruiformes, Charadriiformes and Columbiformes indicates that its presence is primitive. Zusi \& Jehl (1970) report that the foramen is present in all plovers except Charadrius melanops. But the foramen is present in one of the four specimens of this species that I

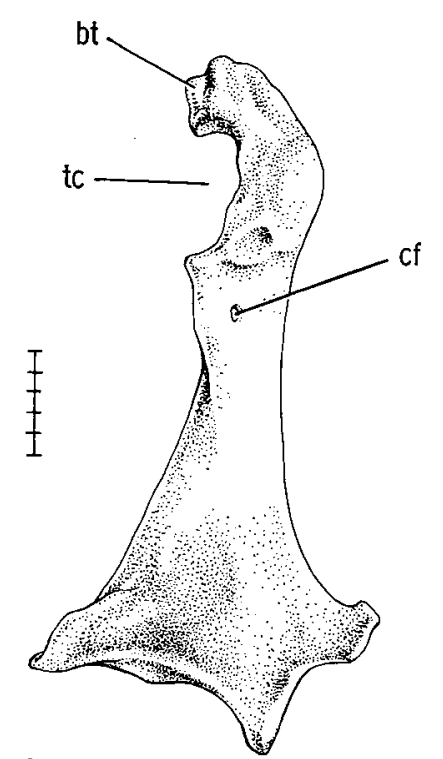

A

FIG. 21. Posterior view of the coracoids of three species. A. Cepphus grylle (UMMZ 151779); B. Thinornis novaeseelandiae (NZNM 1403); C. Nycticryphes semicollaris (UMMZ 157021). Abbreviations: bt, brachial tuberosity; cf, coracoidal foramen; tc, triosseal canal. Each scale unit represents $1 \mathrm{~mm}$.

examined. The specimen with the foramen is clearly an adult, based on gonad measurements. The other three specimens were collected during the latter part of the breeding season but have small gonads; one is clearly immature, as shown by the unossified skeleton of the tarsometatarsus. Since a series of skeletons of adults will probably show the foramen present in individuals with fully ossified skeletons, I scored this species as having a coracoidal foramen.

The coding of this character in the Glareolidae was difficult. The foramen is present in Glareola but absent in Cursorius. In Rhinoptilus the foramen is present in two skeletons of cinctus, in three of four specimens of chalcopterus, but in only one of four specimens of africanus which I examined. All three species of Rhinoptilus were coded as having the foramen. After preliminary analyses indicated that the species of the three genera cited above form a monophyletic group, the absence of a foramen in Cursorius was coded as a uniquely derived state. 
Coding: primitive: coracoidal foramen present (A) (Fig. 21A, B); derived from primitive: coracoidal foramen absent (B) (Fig. 21C); derived from primitive: coracoidal foramen absent in Glareolidae (C) (not illustrated).

Character 40: brachial tuberosity of coracoid (Fig. 21)

In most of the Charadriiformes the brachial tuberosity of the coracoid roofs the triosseal
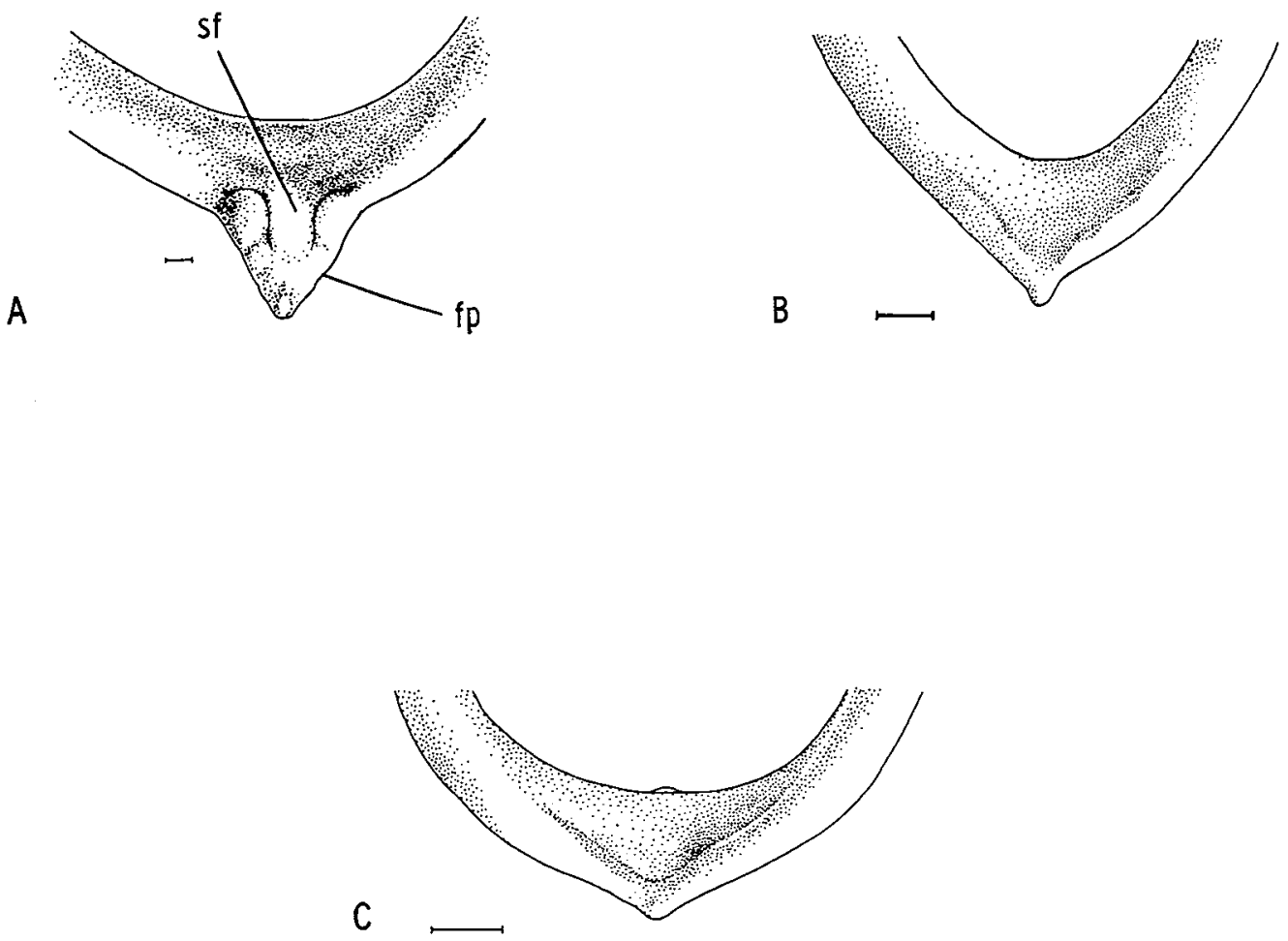

D

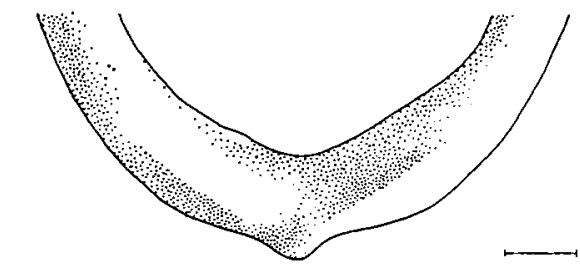

FIG. 22. Anterior view of the furculae of four species. A. Stercorarius pomarinus (UMMZ 153518); B. Pluvianus aegyptius (FMNH 93450); C. Charadrius placidus (USNM 318989); D. Thinornis novaeseelandiae (NZNM 1403). Abbreviations: fp, furcular process; sf, symphysis of furcula. Scales represent $1 \mathrm{~mm}$. 
canal; it is probably the primitive condition. In the Alcidae, however, the head of the coracoid is twisted ventrally so that the tuberosity no longer roofs the canal.

Coding: primitive: brachial tuberosity of coracoid roofing triosseal canal (A) (Fig. $21 \mathrm{~B}, \mathrm{C})$; derived: brachial tuberosity twisted, not roofing canal (B) (Fig. 21A).

Character 41: symphysis of furcula (Fig. 22)

The outward-facing surface of the symphysis of the furcula in Charadriiformes usually has no distinct grooving, or it has a very shallow groove which does not extend into the furcular process. This is believed to be the primitive condition. In the Laroidea a distinct groove extends into the furcular process, while in some plovers and auks there is a distinct ridge at the bottom of the groove.

Coding: primitive: no groove or only a very shallow groove on outward-facing surface of symphysis of furcula (A) (Fig. 22B, D); derived from primitive: distinct groove extending into furcular process (B) (Fig. 22A); derived from primitive: distinct ridge at bottom of groove (C) (Fig. 22C).

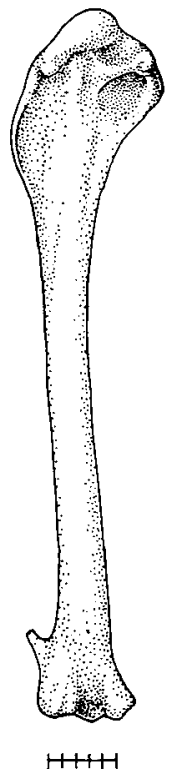

A

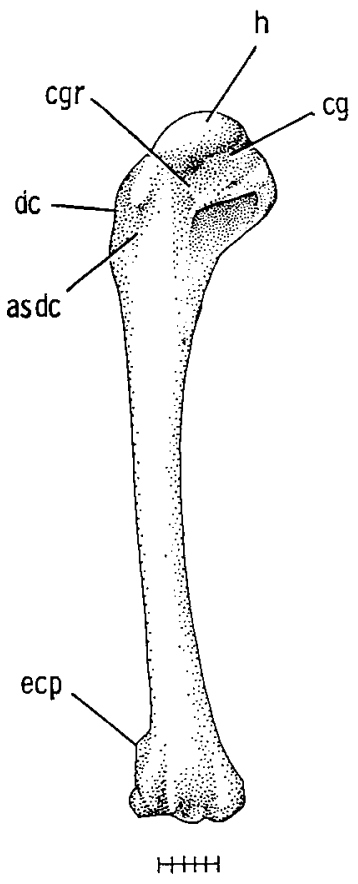

B

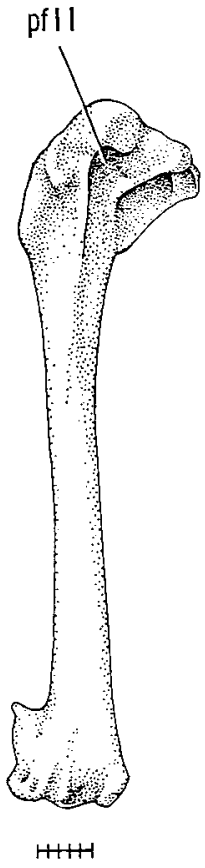

C

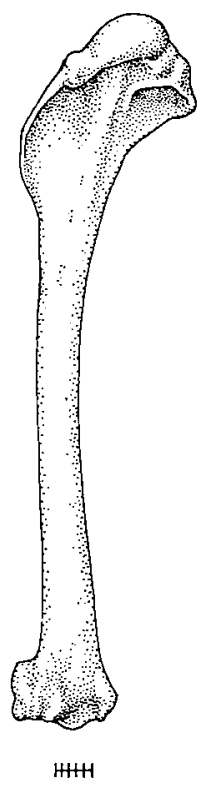

D

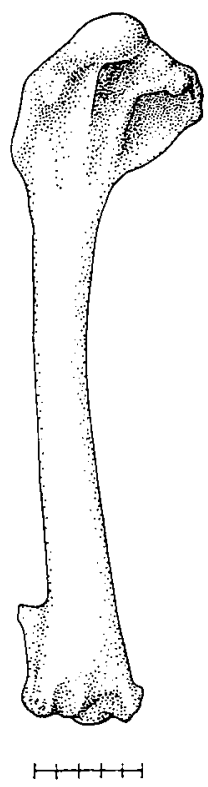

$\mathrm{E}$

FIG. 23. Anconal view of the humeri of five species. A. Rhinoptilus chalcopterus (UMMZ 156673); B. Metopidius indicus (UMMZ 219843); C. Catoptrophorus semipalmatus (UMMZ 156426); D. Burhinus magnirostris (UMMZ 214183); E. Thinornis novaeseelandiae (NZNM 1403). Abbreviations: asdc, anconal surface of deltoid crest; cg, capital groove; cgr, capital groove ridge; dc, deltoid crest; ecp, ectepicondylar prominence; h, head of humerus; pfII, pneumatic fossa II. Each scale unit represents $1 \mathrm{~mm}$. 
Character 42: ridge in capital groove of humerus (Fig. 23)

Some charadriiforms have a ridge in the capital groove of the humerus. Absence of the ridge is probably the primitive state, since no ridge is found in the Gruiformes, most Charadriiformes, and the Columbiformes.

Coding: primitive: no ridge in capital groove of humerus (A) (Fig. 23B, C); derived: ridge present in capital groove of humerus (B) (Fig. 23A, D, E).

Character 43: shape of the deltoid crest of the humerus (Fig. 23)

Zusi \& Jehl (1970) observed that the shape of the anconal surface of the deltoid crest is concave in plovers and convex in sandpipers. Since the surface is convex in the Gruiformes, some Charadriiformes and the Columbiformes, this condition is probably the primitive condition.

Coding: primitive: anconal surface of deltoid crest of humerus convex (A) (Fig. 23B, C); derived: anconal surface of deltoid crest of humerus concave (B) (Fig. 23A, D, E).

Character 44: pneumatic fossa II of humerus (Fig. 23)

A well-developed pneumatic fossa II of the humerus is characteristic of the Charadriiformes and presumably represents the primitive condition.

Coding: primitive: pneumatic fossa II of humerus well-developed (A) (Fig. 23C, D, E); derived: pneumatic fossa II of humerus poorly-developed (B) (Fig. 23A, B).

Character 45: ectepicondylar prominence of humerus (Fig. 23)

Almost all charadriiform birds have a well-developed ectepicondylar prominence on the humerus. This prominence was absent from the humeri of all the members of the Gruiformes and Columbiformes examined. Since the prominence is so widespread in the Charadriiformes and since its absence is correlated with other derived states, presence of the prominence is probably the primitive state of the order.

Coding: primitive: well-developed ectepicondylar prominence on humerus (Fig. 23A, C, E); derived: ectepicondylar prominence absent (A) (Fig. 23B, D).

\section{Character 46: extensor process of the metacarpus (not illustrated)}

The extensor process of the metacarpus of the carpometacarpus is unmodified in the Gruiformes, most Charadriiformes, and the Columbiformes. Some species of Charadriiformes, on the other hand, have well-developed wing spurs or horny knobs on the process, the function of which has never been demonstrated. Several authors have debated the value of wing spurs in classifying the Vanellinae (Boetticher, 1954; Bock, 1958; Wolters, 1974). The wing spurs of plovers and jacanas are well known (Rand, 1954), but several less spectacular modifications of the process have been overlooked. I found a distinct wing knob in Pluvianellus socialis, Chionis alba, and Pluvianus aegyptius, and a possible knob in some plovers and oystercatchers. Sharpe (1896) reported a wing spur for Chionis minor.

The coding of this character was modified during the course of the study. The spurs of jacanas and plovers are quite different (Rand, 1954), those of the former being short cones while those of the latter are more claw-like and somewhat flattened. These two types of 
spurs appear to be independently derived and were coded thus. The jacanas which do not have well-developed spurs all have wing knobs and highly modified radii (Rand, 1954). Since the evolutionary relationship between the jacana knobs and spurs is unclear, an unspecified ancestral process for the jacanas has been assumed; this could logically be an unmodified process, a knob, or a spur; it assumes an unmodified radius which gave rise to two independently derived conditions: a well-developed spur with an unmodified radius and a wing knob with a modified radius. The wing knobs in Pluvianellus, Chionis and Pluvianus were originally coded as the same state. Later it became clear that evidence from other characters indicates that the knob in Pluvianus has been independently derived, and the character coding, was changed accordingly. In addition, other evidence indicates that Hoploxypterus cayanus is not a member of the Vanellinae and that its wing spur has been derived independently of that found in the lapwings. Coding was changed to reflect this conclusion. Finally, the low, flat process found in some of the Alcidae is probably an independent derivation from the primitive state.

Coding: primitive: unmodified extensor process of metacarpus of carpometacarpus (A); derived from primitive: wing knob of Pluvianus (B); derived from primitive: wing knobs of Chionis and Pluvianellus (I); derived from primitive: wing spurs of Vanellinae (C); derived from primitive: wing spur of Hoploxypterus $(\mathrm{F})$; derived from primitive: low, flat process of Alcidae (D); derived from primitive: hypothetical ancestral state for Jacanidae $(G)$; derived from $(G)$ : jacana wing spurs $(E)$; derived from $(G)$ : jacana wing knobs (H).
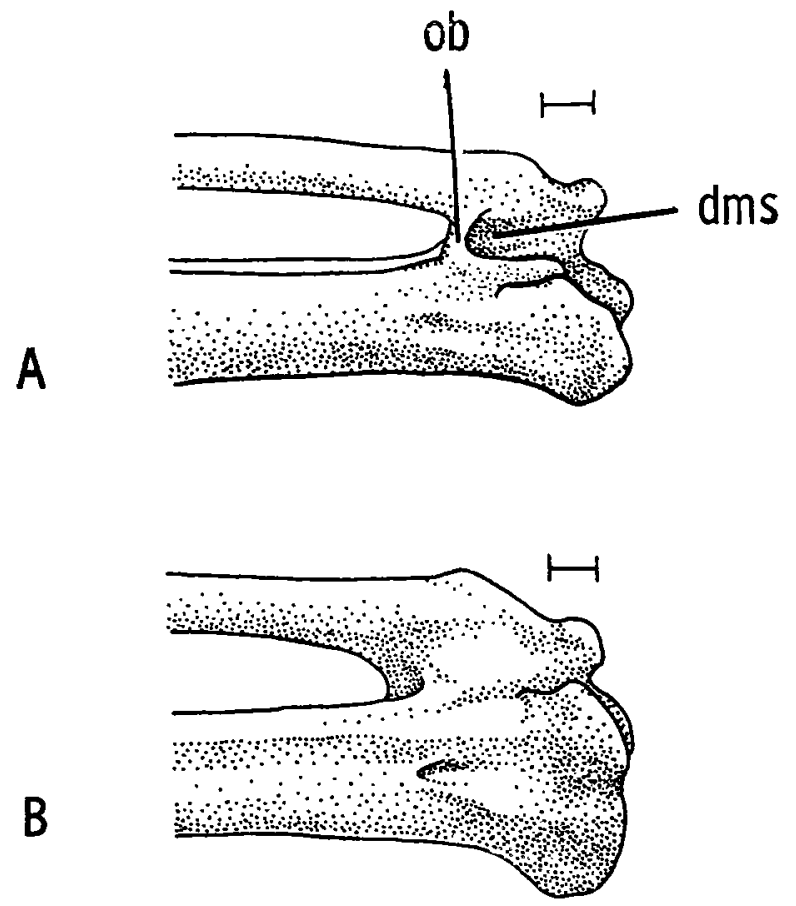

FIG. 24. Dorsal view of the distal end of carpometacarpi of two species. A. Metopidius indicus (UMMZ 219846); B. Vanellus macropterus (RVNH b). Abbreviations: dms, distal metacarpal symphysis; os, ossified bridge. Scales represent $1 \mathrm{~mm}$. 
Character 47: position of intermetacarpal tuberosity on metacarpal II of carpometacarpal (not illustrated)

The intermetacarpal tuberosity is a large scar on the edge of the dorsal surface of metacarpal II of the carpometacarpus facing into the intermetacarpal space. This is the location of attachment for M. flexor metacarpi posterior (George \& Berger, 1966). The most widespread and presumably primitive state for this character is for the scar to be located outside the groove where metacarpals II and III join.

Coding: primitive: intermetacarpal tuberosity outside groove where metacarpals II and III join (A); derived: intermetacarpal tuberosity within groove formed where metacarpals II and III join (B).

Character 48: distal metacarpal symphysis of carpometacarpus (Fig. 24)

A small ossified bridge over the distal metacarpal symphysis of the carpometacarpus is unique to the Jacanidae and is probably a derived condition.

Coding: primitive: no ossified bridge over the distal metacarpal symphysis of the carpometacarpus (A) (Fig. 24B); derived: bridge present (B) (Fig. 24A).

Character 49: proximal phalanx, digit III (not illustrated)

A strongly perforated proximal phalanx, digit III, is unique to the Laroidea within the Charadriiformes; it probably represents a derived condition. Some specimens of the Phalaropodidae and the Glareolidae seem to have the derived state, but in all cases the perforation is a hole in a thin sheet of bone and appears to represent incomplete ossification or a damaged specimen. The thin sheet of bone is always absent in the Laroidea. The states of this character are well-illustrated by Stegmann (1963).

Coding: primitive: proximal phalanx, digit III, not perforated (A); derived: proximal phalanx, digit III, perforated (B).

\section{Synsacrum characters}

\section{Character 50: synsacral strut (Figs 25, 26)}

Most charadriiform birds have a strut or brace extending from the fused sacral-caudal vertebrae to the acetabulum. In the most widely distributed and presumably primitive state for the Charadriiformes this strut arises from the fifth vertebra from the posterior end of the synsacrum. In the derived states the strut lies one vertebra proximal or distal to the end of the synsacrum or is absent.

Coding: primitive: synsacral strut extending from fifth vertebra (A) (Figs 25, 26); derived from primitive: synsacral strut extending from sixth vertebra (B) (not illustrated); derived from primitive: synsacral strut extending from fourth vertebra (D) (not illustrated); derived from primitive: synsacral strut absent (C) (not illustrated).

\section{Character 51: second synsacral strut (not illustrated)}

A few species of Charadriiformes have a second synsacral strut either posterior or anterior to the first strut. The value of this character is doubtful because the second strut is present in some specimens of several species but absent in other specimens. Some specimens also have an additional strut on one side but not on the other.

Coding: primitive: no second synsacral strut (A); derived from primitive: second strut posterior to first strut (B); derived from primitive: second strut anterior to first strut $(C)$. 


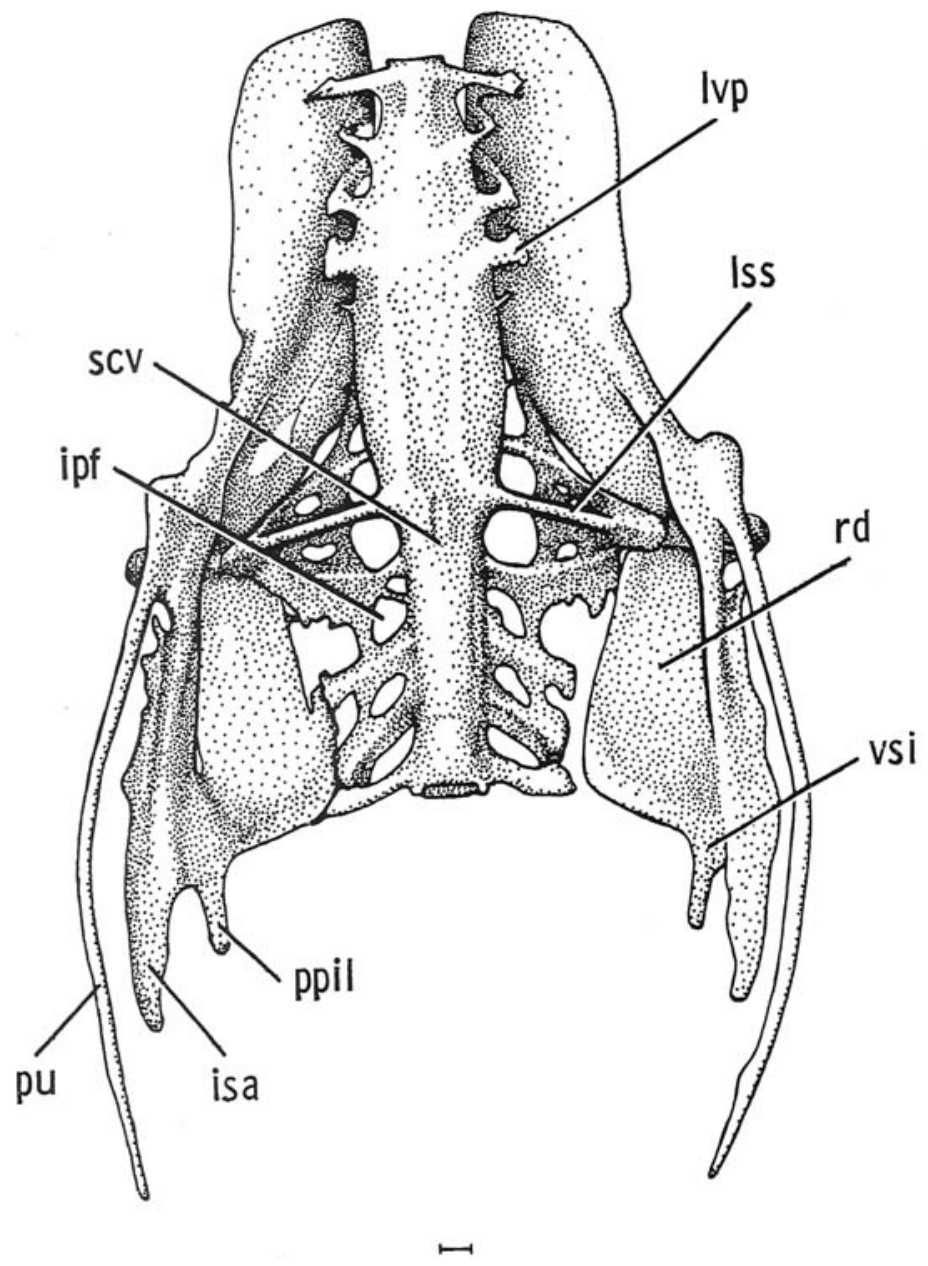

FIG. 25. Ventral view of the synsacrum of Thinomis novaeseelandiae (NZNM 1403). Abbreviations: ipf, interapophyseal foramen; isa, ischial angle; lss, lateral synsacral strut; lvp, lumbar vertebral parapophysis; ppil, posterior projection of ilium; pu, pubis; rd, renal depression; scv, sacral-caudal vertebrae; vsi, ventral surface of ilium. Scale represents $1 \mathrm{~mm}$.

Character 52: position of lateral synsacral strut (Figs 25, 26)

The lateral synsacral strut extends from the fused sacral-caudal vertebrae to the acetabulum in the Gruiformes and most of the Charadriiformes but falls short of the acetabulum in the Columbiformes. The most widely distributed condition within the Charadriiformes, in which the strut extends to the acetabulum, is probably primitive; The condition in the Alcidae, in which the strut is completely absent, is probably an independently derived state.

Coding: primitive: lateral synsacral strut extending from fused sacral-caudal vertebrae to acetabulum (A) (Figs 25, 26); derived from primitive: strut falling short of acetabulum (B) (not illustrated); derived from primitive: strut absent (C) (not illustrated). 


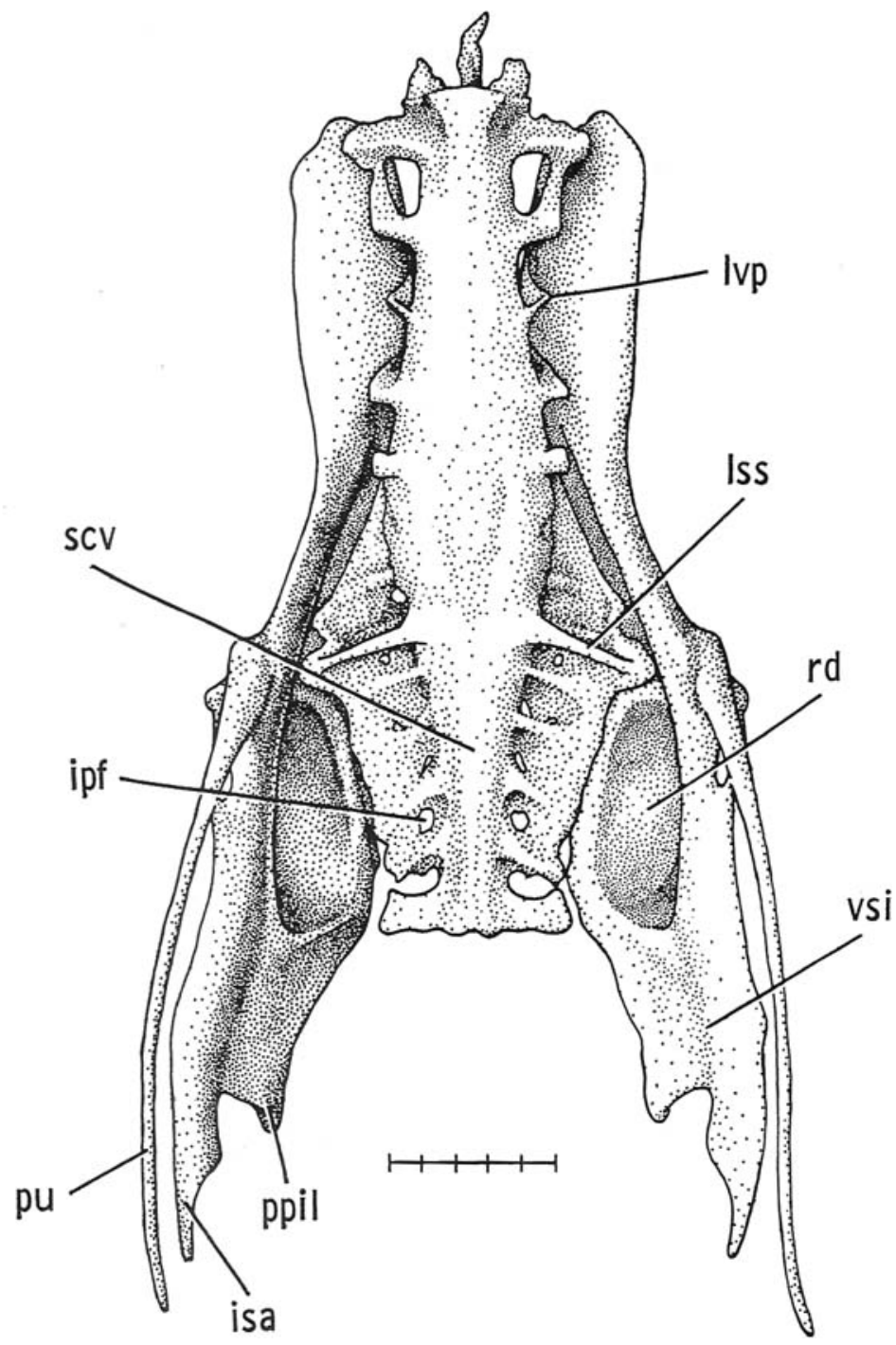

FIG. 26. Ventral view of the synsacrum of Metopidius indicus (UMMZ 214551). Abbreviations as in Fig. 25. Scale represents $1 \mathrm{~mm}$.

Character 53: number of lumbar vertebral parapophyses (Figs 25, 26)

Most charadriiform birds have three parapophyses on the lumbar vertebrae; this is probably the primitive state for the order.

Coding: primitive: three parapophyses on lumbar vertebrae (A) (Figs 25, 26); derived: four parapophyses on lumbar vertebrae (B) (not illustrated).

Character 54: condition of posterior end of the renal depression (Figs 25, 26)

The posterior end of the renal depression of the synsacrum of most charadriiform birds is almost flat, and there is little or no discontinuity between the end of the depression and 
the ventral surface of the ilium; this condition is probably the primitive state. In some species the end of the depression is abrupt, deep, and partially roofed by the inner posterior surface of the ilium; in others a strut defines the end of the depression.

Coding: primitive: posterior end of the renal depression flat with little or no discontinuity between end of depression and ventral surface of ilium (A) (Fig. 25); derived from primitive: distinct strut at end of depression (B) (not illustrated); derived from primitive: end of depression deep and abrupt (C) (Fig. 28).

\section{Character 55: foramina on ventral surface of ilium (Figs 25, 26)}

There are foramina on the ventral surface of the ilium (= renal bar of Holmann (1961)?) in some species of Stercorarius. While some species of gulls and terns have depressions in this area, the foramina are found only in the skuas.

Coding: primitive: no foramina in ventral surface of ilium (A) (Figs 25, 26); derived: foramina present in ventral surface of ilium (B) (not illustrated).

Character 56: interapophyseal foramina of synsacrum (Figs 25, 26)

A large number of interapophyseal foramina is characteristic of the charadriiform synsacrum. Almost all the Gruiformes and Columbiformes have two or fewer such foramina on each side of the synsacrum. In the Charadriiformes only two species of Jacanidae and the woodcocks consistently have fewer than three foramina on each side of the synsacrum, a condition which is probably derived.

Coding: primitive: three or more interapophyseal foramina on each side of synsacrum (A) (Figs 25, 26); derived: fewer than three foramina (B) (not illustrated).

Character 57: shape of the posterior medial edge of the ilium (Figs 25, 26)

The posterior edge of the ilium of most Charadriiformes meets its medial edge at nearly a right angle. In some species the edge forms a smooth curve, while in others the medial edge is straight all the way to the ischial angle.

Coding: primitive: posterior medial and medial edge of ilium forming abrupt right angle (B) (Fig. 25); derived from primitive: posterior medial and medial edge of ilium forming smooth curve (A) (Fig. 26); derived from primitive: medial edge of ilium straight all the way to ischial angle (C) (not illustrated).

\section{Character 58: extent of the ischium (Fig. 27)}

In most Charadriiformes the ischial angle extends posterior to the posterior projection of the ilium, often as far as the end of the pubis. This is probably the primitive condition.

Coding: primitive: ischial angle extending posterior to posterior projection of ilium (A) (Fig. 27B); derived: ischial angle relatively short and about even with posterior projection of ilium (B) (Fig. 27).

Character 59: fusion of the ischium and pubis (Fig. 27)

In most Charadriiformes the ischium and pubis are not fused or are fused only near their posterior ends. This is probably the primitive condition. In some species, however, the ischium and pubis are fused immediately posterior to the obturator foramen. Both states can be found in specimens of some species. 

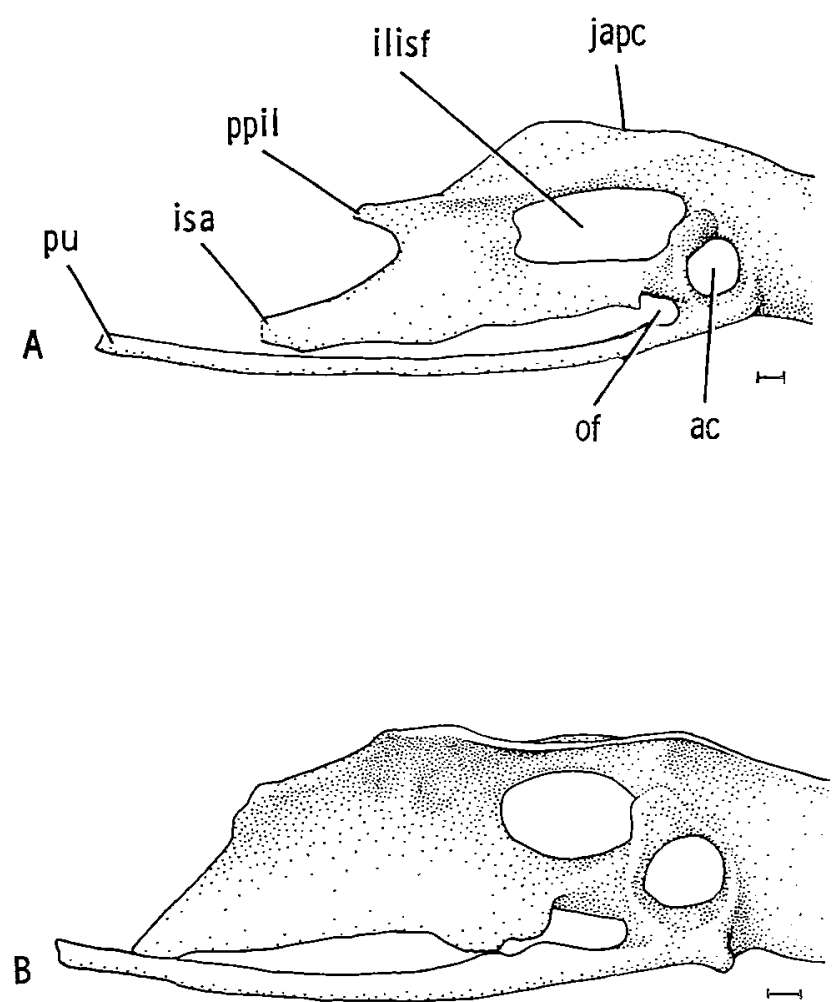

Fig. 27. Lateral view of the synsacra of two species. A. Thinornis novaeseelandiae (NZNM 1403); B. Metopidius indicus (UMMZ 214551). Abbreviations: ac, acetabulum; ilisf, ilioischiatic fenestra; isa, ischial angle; japc, junction of anterior and posterior crests; of, obturator foramen; ppil, posterior projection of ilium; pu, pubis. Scales represent $1 \mathrm{~mm}$.

Coding: primitive: ischium and pubis not fused immediately behind obturator foramen (A) (Fig. 27A, B); derived: ischium and pubis fused immediately posterior to obturator foramen (B) (not illustrated).

Character 60: shape of junction of anterior and posterior crests of the pelvis (Fig. 27)

In the most widespread and presumably primitive condition for the Charadriiformes the junction of the anterior and posterior crests of the pelvis is smooth and rounded. In some species the crests form a distinct projection over the ilioischiatic fenestra.

Coding: primitive: junction of anterior and posterior crests of pelvis smooth and rounded (A) (Fig. 27B); derived: junction of anterior and posterior crests of pelvis forming a distinct projection over ilioischiatic fenestra (B) (Fig. 27A).

\section{Hind limb characters}

Character 61: relative positions of trochlea for digits II and IV of the tarsometatarsus (Fig. 28A, B, C)

I found three patterns for the relative positions of the trochlea for digits II and IV: either they are equally and only slightly elevated compared to the trochlea for digit III, 


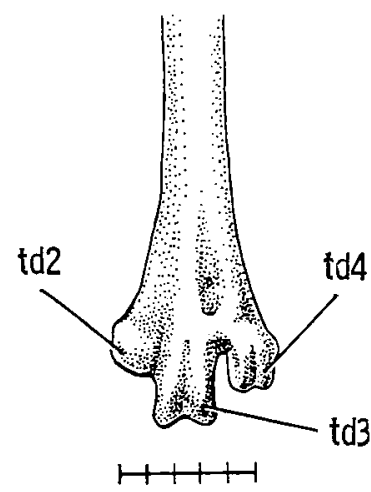

A
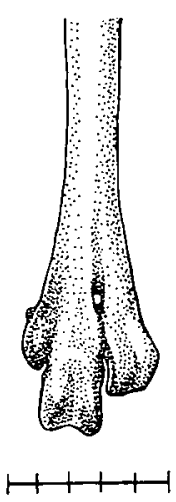

B
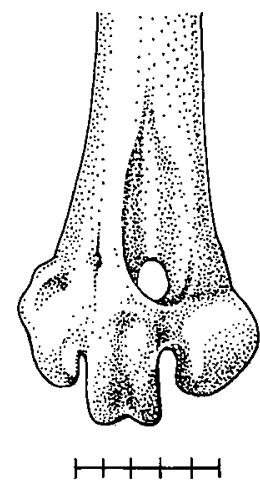

C

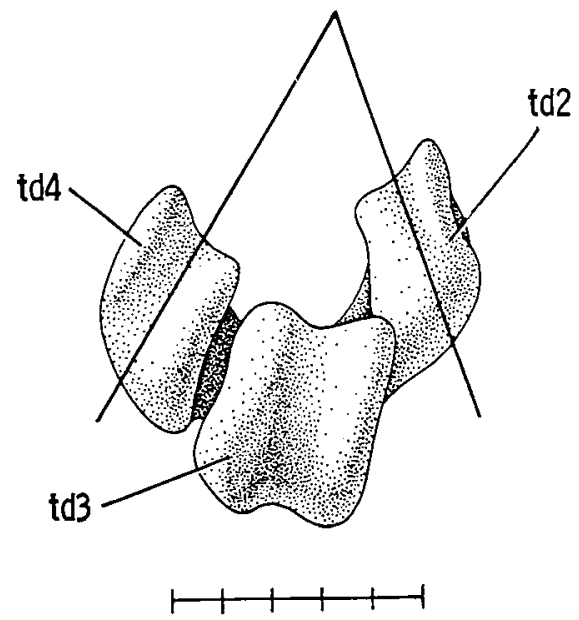

$D$

FIG. 28. View of the distal end of the tarsometatarsi of four species. Anterior view: A. Aphriza virgata (UMMZ 291182); B. Thinornis novaeseelandiae (NZNM 1403); C. Metopidius indicus (UMMZ 214551). Ventral view: D. Cladorhynchus leucocephala (UMMZ 214181). Abbreviations: td2, trochlea for digit II; td3, trochlea for digit III; td4, trochlea for digit IV. Each scale unit represents $1 \mathrm{~mm}$. 
or they are both elevated with that for digit II slightly more so than that for digit IV, or they are both considerably elevated with that for digit II much more so than that for digit IV. The distribution of the states among the Gruiformes, Charadriiformes and Columbiformes indicates that the condition in which the trochlea for digit II is slightly elevated is probably the primitive state.

Coding: primitive: trochlea for digits II and IV elevated compared to that for digit II, trochlea for digit II only slightly more elevated than that for digit IV (A) (not illustrated); derived from primitive: trochlea for digits II and IV considerably elevated compared to that for digit III, trochlea for digit II more so than that for digit IV (B) (Fig. 28A, B); derived from primitive: trochlea for digits II and IV only slightly elevated compared to that for digit III (C) (Fig. 28C).

\section{Character 62: hallux (not illustrated)}

Most Gruiformes, Charadriiformes and Columbiformes have a well-developed hallux; this is probably the primitive state.

Coding: primitive: hallux present (A); derived: hallux absent (B).

Character 63: angle formed by trochleae for digits II and IV (Fig. 28D)

The angles formed between the trochleae for digits II and IV fall into three groups: those larger than $35^{\circ}$, those between $35^{\circ}$ and $25^{\circ}$, and those smaller than $25^{\circ}$. The middle group appears to be the primitive state, based on its distribution within the Charadriiformes.

Coding: primitive: angle between $35^{\circ}$ and $25^{\circ}$ (A) (not illustrated); derived from primitive: angle larger than $35^{\circ}$ (B) (Fig. 28D); derived from primitive: angle smaller than $25^{\circ}$ (C) (not illustrated).

Characters 64, 65, 66, 67, 68, 69, 70: hypotarsus (Fig. 29)

The pattern of tendinal canals in the hypotarsus is complex and offers abundant comparative material for phylogenetic and functional studies. While the patterns of muscles and tendons in bird legs have received considerable attention, little has been published on the patterns of the passage of the tendons through and around the hypotarsus. Storer's (1963) use of the hypotarsus in inferring relationships among the grebes represents one of the new systematic uses of these characters.

The arrangement of the tendons in the hypotarsus of the Blue grouse, Dendragapus obscurus (Hudson, Lanzillotti \& Edwards, 1959), and for the Common crow, Corvus brachyrhynchos (Hudson, 1937), were used to estimate which tendon is associated with the tendinal canals of charadriiform hypotarsi. The hypothetical charadriiform hypotarsus illustrated in Fig. 29E shows the relative locations of six tendons, which could account for all the patterns found. The assumed homologies for each tendon are as follows: No. 1, tendon for M. flexor digitorum longus. George \& Berger (1966) state that this tendon is the only one which traverses a bony canal in Totanus $(=$ Tringa $)$ and Larus; No. 2 , tendon of $M$. flexor perforatus digiti IV and/or tendon for $M$. flexor perforans et perforatus digiti II; No. 3, tendon of M. flexor hallucis longus (In species with a very small or no hallux this tendon has been found to fuse with the tendon of M. flexor digitorum longus (George \& Berger, 1966).); No. 4, tendon for M. peronaeus longus; No. 5, tendon for $M$. flexor perforatus digiti IV; No. 6 , tendon for M. flexor perforans et perforatus 


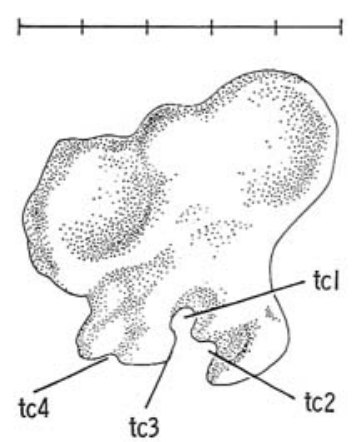

A

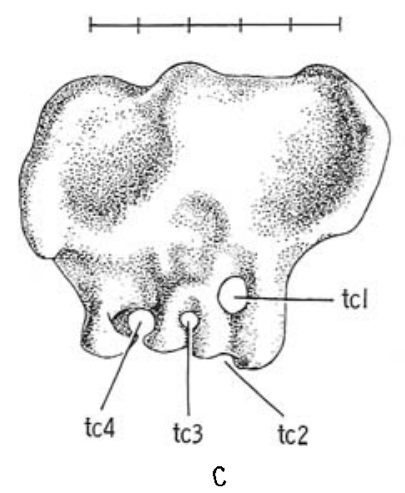

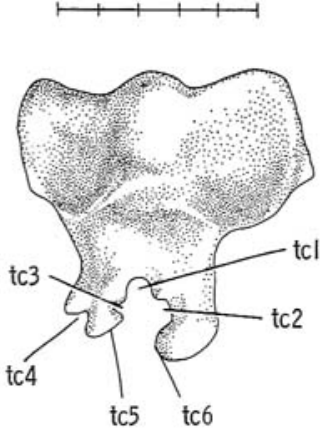

B

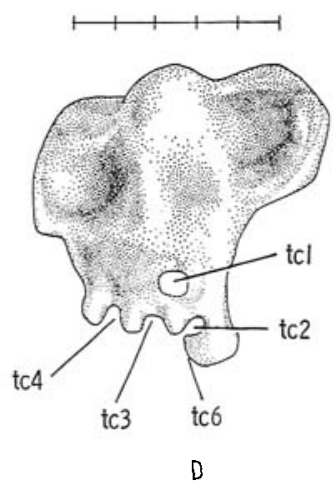

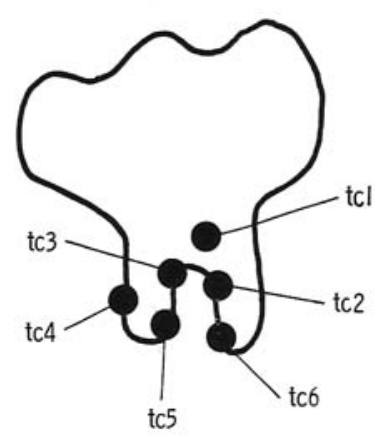

E

FIG. 29. View of the proximal end of the tarsometatarsi of four species. A. Thinomis novaeseelandiae (NZNM 1403); B. Cladorhynchus leucocephala (UMMZ 214181); C. Cepphus grylle (UMMZ 151779); D. Scolopax rusticola (UMMZ 156165); E. Hypothetical hypotarsus showing the six possible tendinal canals. Abbreviations: tcl-6, tendinal canals of hypotarsus No. 1-6. Each scale unit represents $1 \mathrm{~mm}$.

digiti III. There is a canal for a tendon lying between tendons 2 and 3 in Rhinoptilus chalcopterus, $R$. cinctus, and some specimens of Haematopus. This condition may represent a separation of the pathways of the tendons assumed to pass through No. 1.

Character 64: tendinal canal No. 1 (Fig. 29). In most charadriiform birds a bony canal is present for this tendon; the condition in which only a groove is present is probably derived.

Coding: primitive: tendinal canal No. 1 a bony canal (B) (Fig. 29C, D); derived: tendinal canal No. 1 a groove (A) (Fig. 29A, B).

Character 65: location of tendinal canal No. 1 (Fig. 29). The canal for tendon No. 1 is found either directly anterior to the canal for tendon No. 2, anterior to the space between the canals for tendons No. 2 and 3, or directly anterior to the canal for tendon No. 3. The distribution of the states for this character within the Charadriiformes indicates that the primitive condition is probably for the canal for tendon No. 1 to be directly anterior to the canal for tendon No. 2.

Coding." primitive: canal for tendon No. 1 directly anterior to canal for tendon No. 2 
(B) (Fig. 29C, D); derived from primitive: canal for tendon No. 1 anterior to space between canals for tendons No. 2 and 3 (A) (not illustrated); derived from (A): canal for tendon No. 1 directly anterior to canal for tendon No. 3 (C) (Fig. 29A, B).

Character 66: tendinal canal for tendon No. 3 (Fig. 29). The canal for this tendon is either a bony canal, a groove, or is undetectable on the surface of the bone. The state in which only a groove is present is probably the primitive state for the order.

Coding: primitive: canal for tendon No. 3 a groove (A) (Fig. 29A, B, D); derived from primitive: canal for tendon No. 3 a bony canal (B) (not illustrated); derived from primitive: canal for tendon No. 3 a bony canal in Alcidae (D) (Fig. 29C); derived from primitive: canal for tendon No. 3 not detectable on bone (C) (not illustrated).

Character 67: tendinal canal for tendon No. 4 (Fig. 29). The description of this character is the same as for the canal for tendon No. 3 .

Coding: primitive: canal for tendon No. 4 a groove (A) (Fig. 29A, B, C, D); derived from primitive: canal for tendon No. 4 a bony canal (C) (not illustrated); derived from primitive: canal for tendon No. 4 not detectable on bone (B) (not illustrated).

Character 68: tendinal canal for tendon No. 6 (Fig. 29). A canal for this tendon is not detectable on the bones of most Charadriiformes; this is probably the primitive state.

Coding: primitive: no canal for tendon No. 6 detectable on bone (A) (Fig. 29A, C); derived from primitive: canal a groove on bone for tendon No. 6 (B) (Fig. 29B, D).

Character 69: tendinal canal for tendon No. 5 (Fig. 29). Like the canal for tendon No. 6 , the canal for this tendon is usually not detectable on the bone. Grooves are found only in the Vanellinae and Recurvirostridae.

Coding: primitive: no canal for tendon No. 5 detectable on bone (A) (Fig. 29A, C, D); derived from primitive: canal a groove for tendon No. 5 (B) (Fig. 29B).

Character 70: alignment of tendinal canals No. 2, 3, and 4 (Fig. 29). The derived state for this character is the condition found in the snipes and woodcocks, in which the canals for tendons No. 2, 3, and 4 lie on a straight line passing through the centres of canals No. 2 and 4.

Coding: primitive: canals for tendons No. 2, 3, and 4 not on a straight line (A) (Fig. 29A, B, C); derived: canals for tendons No. 2, 3, and 4 on straight line passing through centres of canals No. 2 and 4 (B) (Fig. 29D).

\section{RESULTS}

\section{The Charadriiformes}

The compatibility analysis of the 70 characters and 227 EUs in this study yielded one largest clique of 23 characters:

Charadriiformes Clique: 1, 7, 9, 13, 14, 17, 19, 21, 22, 26, 33, 37, 39, 40, 42, 43, 46, $48,49,52,53,55,70$.

The tree defined by this clique (Fig. 30) shows that the Charadriiformes form three monophyletic lines which I shall call suborders: the Scolopaci (Jacanidae, Rostratulidae, Scolopacidae, Phalaropodidae, and Thinocoridae: nodes bb, cu, do, go, j1, j2, pe, s1, s2, sc, t1, and t2); the Charadrii (Stercorariidae, Laridae, Dromadidae, Glareolidae, Burhinidae, Chionididae, Charadriidae, Haematopodidae, Ibidorhyncha and Recurvirostridae: nodes ch, co, ep, gt, hc, ja, mp, po, sb, tk, v1, v2, and wb); and the Alcidae (nodes a1 and a2). 


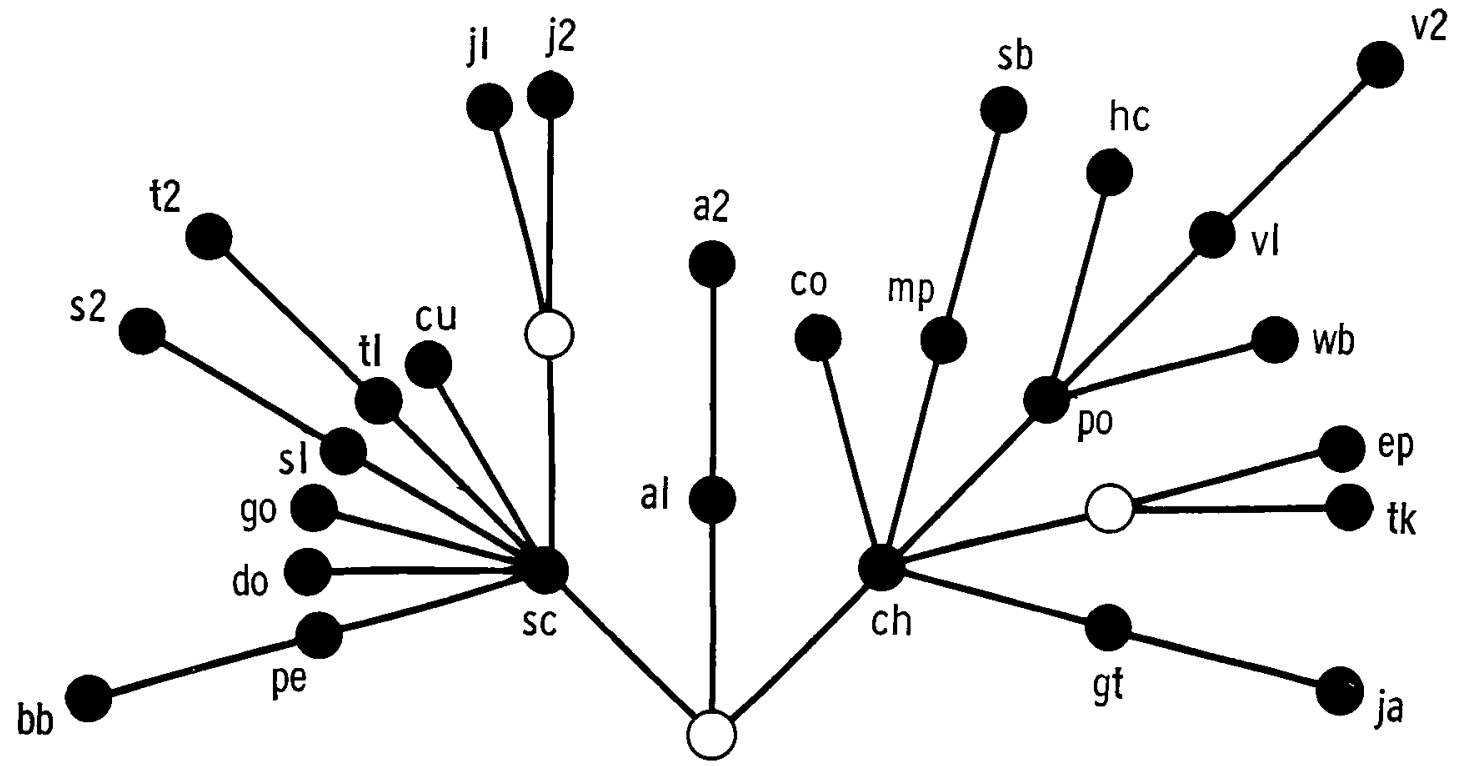

FIG. 30. Preliminary estimate of the phylogeny of the Charadriiformes. The tree defined by the 23 primary characters for the order. Abbreviations: al, Endomychura; a2, Cepphus, Uria; bb, Limicola; ch, Dromas, Glareolidae (part); co, Cursorius; cu, Prosobonia, Bartramia, Numenius; do, Limnodromus; ep, Pluvianus; go, Limosa; gt, Stercorariidae (part), Laridae, Rynchops; hc, Hoploxypterus; j1, Jacanidae (part); j2, Jacanidae (part); ja, Stercorariidae (part); mp, Pluvianellus; pe, Actitis, Aphriza, Calidris, Eurynorhynchus, Micropalama, Tryngites, Philomachus; po, Peltohyas, Charadriinae (part), Haematopus, Ibidorhyncha, Recurvirostridae; 1 , Tringa (part), Catoptrophorus, Heteroscelus; t2, Tringa (part); tk, Burhinidae; s1, Coenocorypha; s2, Lymnocryptes, Gallinago, Philohela, Scolopax; sc, Phalaropodidae, Thinocoridae, Rostratulidae, Xenus, Arenaria; v1, Vanellinae (part); v2, Vanellinae (part); wb, Anarhynchus.

Since the relationships among the Scolopaci and among the Charadrii are poorly resolved by this analysis, each suborder was analysed separately to find secondarily compatible characters. Subsequently, analyses of smaller and smaller subgroups identified as monophyletic by a previous analysis were made until all locally compatible characters had been found and maximal resolution of relationships had been made. The Alcidae were not analysed further.

\section{The Scolopaci}

\section{The suborders of the Charadriiformes}

The compatibility analysis of the 54 characters which vary among the 87 EUs in this group yielded eight largest cliques of 25 characters each:

Scolopaci Clique A: 1, 3, 4, 6, 8, 9, 17, 18, 21, 22, 23, 24, 26, 28, 44, 45, 46, 47, 48, $52,61,62,66,68,70$

Scolopaci Clique B: 1, 3, 4, 6, 8, 9, 17, 18, 21, 22, 23, 24, 26, 28, 44, 45, 46, 47, 48, $52,61,62,67,68,70$

Scolopaci Clique C: 1, 3, 4, 8, 9, 17, 18, 19, 21, 22, 23, 24, 26, 28, 44, 45, 46, 47, 48, $52,61,62,66,68,70$ 
Scolopaci Clique D: 1, 3, 4, 8, 9, 17, 18, 19, 21, 22, 23, 24, 26, 28, 44, 45, 46, 47, 48, $52,61,62,67,68,70$

Scolopaci Clique E: 1, 3, 4, 6, 8, 9, 13, 17, 21, 22, 23, 24, 26, 28, 44, 45, 46, 47, 48, $52,61,62,66,68,70$

Scolopaci Clique F: 1, 3, 4, 6, 8, 9, 13, 17, 21, 22, 23, 24, 26, 28, 44, 45, 46, 47, 48, $52,61,62,67,68,70$

Scolopaci Clique G: 1, 3, 4, 8, 9, 13, 17, 19, 21, 22, 23, 24, 26, 28, 44, 45, 46, 47, 48, $52,61,62,66,68,70$

Scolopaci Clique H: 1, 3, 4, 8, 9, 13, 17, 19, 21, 22, 23, 24, 26, 28, 44, 45, 46, 47, 48, $52,61,62,67,68,70$.

While this seems like a bewildering set from which to pick a best estimate, the choice is straightforward. Twenty-two characters appear in all the cliques. If one compares pairs of cliques starting with Clique A, both members of a pair are identical except that the first member contains Character 66 while the second contains Character 67. These two characters assort the species of Gallinago and Lymnocryptes between nodes s 2 and s 3 on all the trees defined by these cliques. The choice of Character 66 vs. 67 is discussed elsewhere
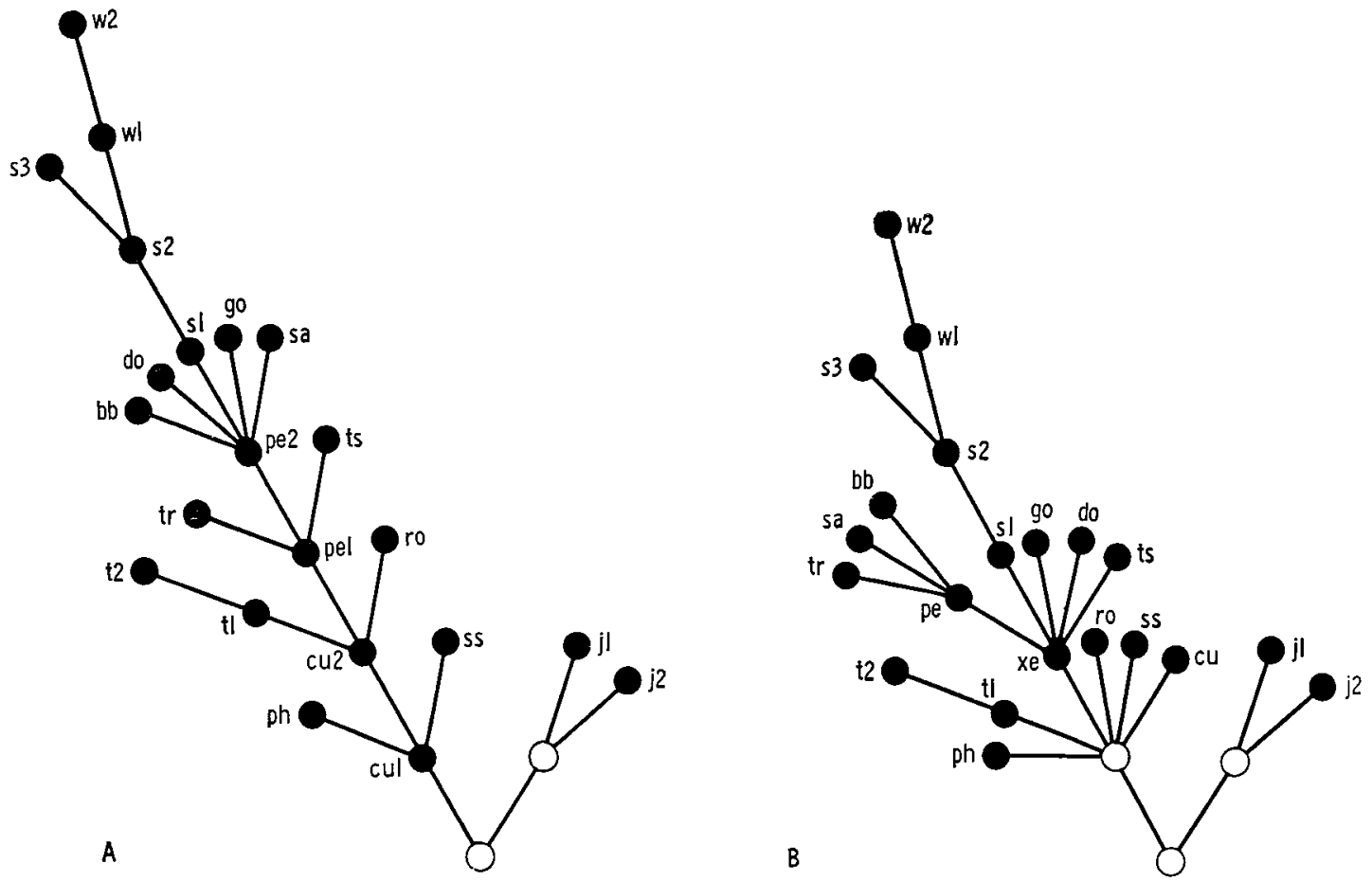

FIG. 31. Two estimates of the phylogeny of the Scolopaci. Abbreviations: bb, Limicola; cu, Prosobonia, Bartramia, Numenius; cul, Prosobonia, Bartramia, Numenius (part); cu2, Numenius (part); do, Limnodromus; go, Limosa; j1, Jacanidae (part); j2, Jacanidae (part); pe, Actitis, Aphriza, Calidris (part), Eurynorhynchus, Micropalama, Philomachus; pe1, Actitis, Aphriza, Calidris (part), Philomachus; pe2, Xenus, Calidris (part), Eurynorhynchus, Micropalama; ph, Phalaropus; ro, Rostratula, Nycticryphes; s1, Coenocorypha; s2, Lymnocryptes (if Character 66 is true), Gallinago (part); s3, Lymnocryptes (if Character 67 is true), Gallinago (part); sa, Calidris alba; ss, Thinocoridae; t1, Tringa (part), Catoptrophorus, Heteroscelus; t2, Tringa stagnatilis; tr, Tryngites; ts, Arenaria; w1, Philohela; w2, Scolopax; xe, Xenus. 
(Strauch, 1976). This leaves a choice among four pairs of cliques; only four remaining characters $(6,13,18$, and 19) do not appear in all the cliques. Of these, only Characters 13 and 19 were included in the largest clique found in the Charadriiformes analysis; thus they have already been found reliable in a more rigorous analysis. On this basis the tree defined by Clique G (Fig. 31B) was chosen as the best estimate of relationships among the Scolopaci. Figure 31A shows the tree defined by Clique A, which includes Characters 6 and 18 .

On Tree B two lines diverge from the ancestor of the suborder. The Jacanidae (nodes $\mathrm{j} 1$ and j2) share derived states of Characters 44,45, 46, 47, 48, 52, and 61, while the Rostratulidae, Scolopacidae, Phalaropodidae, and Thinocoridae (nodes bb, cu, do, go, pe, ph, ro, s1, s2, s3, sa, ss, t1, t2, ts, tr, w1, w2, and xe) share derived states of Characters 24 and 61.

The Rostratulidae, Scolopacidae, Phalaropodidae and Thinocoridae. The compatibility analysis of the 46 characters which vary among the 79 EUs in this assemblage yielded eight largest cliques of 20 characters each. These cliques are identical to the cliques found in the Scolopaci analysis except that Characters $24,44,45,46,47,48,52$, and 61 have been

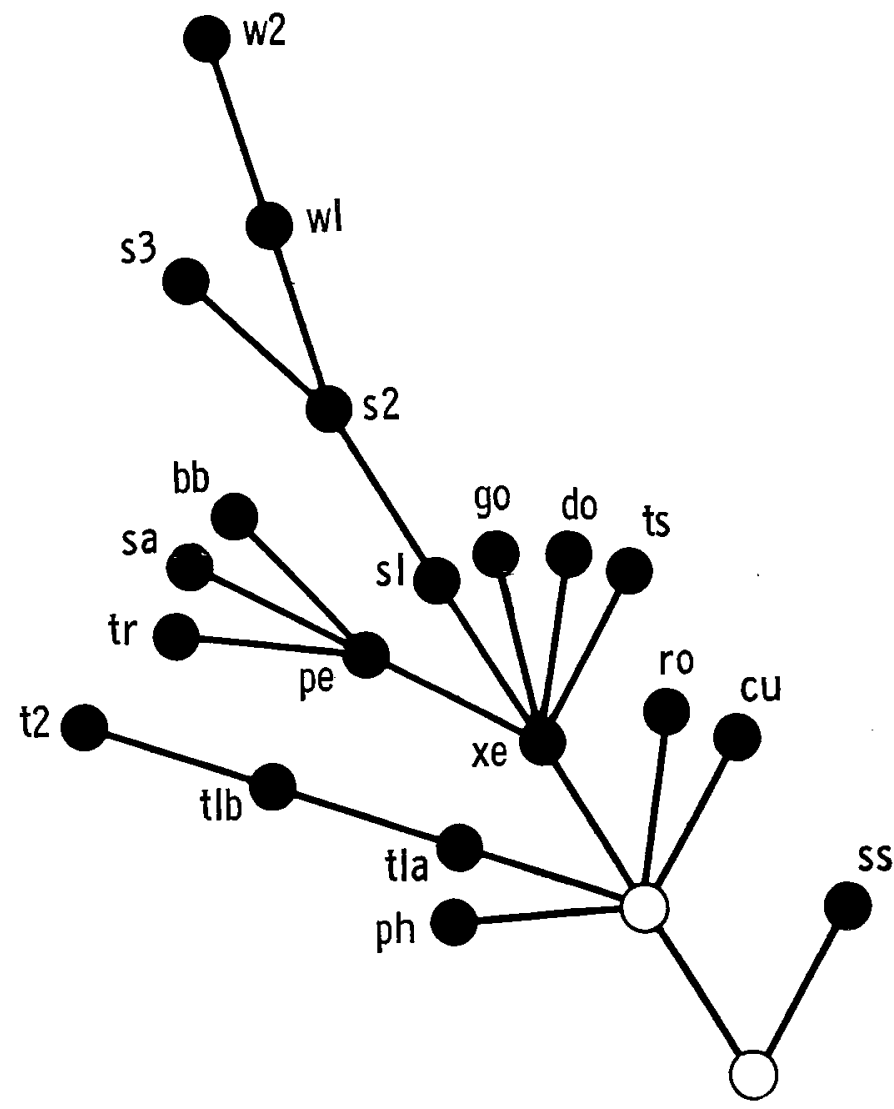

FIG. 32. An estimate of the phylogeny of the Rostratulidae, Scolopacidae, Phalaropodidae, and Thinocoridae. Abbreviations as in Fig. 31 plus t1a, Tringa (part), Catoptrophorus; t1b, Tringa (part), Heteroscelus. 
deleted (since they do not vary among the Scolopacoidea) and Characters 10, 41, and 56 added to each clique. As in the Scolopaci analysis only one pair of cliques includes Characters 13 and 19, and it is thus chosen as the best estimate of relationships in this group. Only the tree defined by Clique D (Fig. 32; compare with Fig. 31) is illustrated. This tree shows that two lines diverge from the common ancestor of this group. The Thinocoridae (node ss) share derived states of Characters 4 and 8, while the Rostratulidae, Scolopacidae, and Phalaropodidae (nodes bb, cu, do, go, pe, ph, ro, s1, s2, tla, t1b, t2, $\mathrm{ts}, \mathrm{tr}, \mathrm{w} 1, \mathrm{w} 2$, and $\mathrm{xe}$ ) share a derived state of Character 10.

The Rostratulidae, Scolopacidae, and Phalaropodidae. The compatibility analysis of the 45 characters which vary among the 76 EUs in this assemblage yielded eight largest cliques of 19 characters each. These cliques are identical to the cliques found in the previous analysis except that Character 10 has been deleted from each clique.

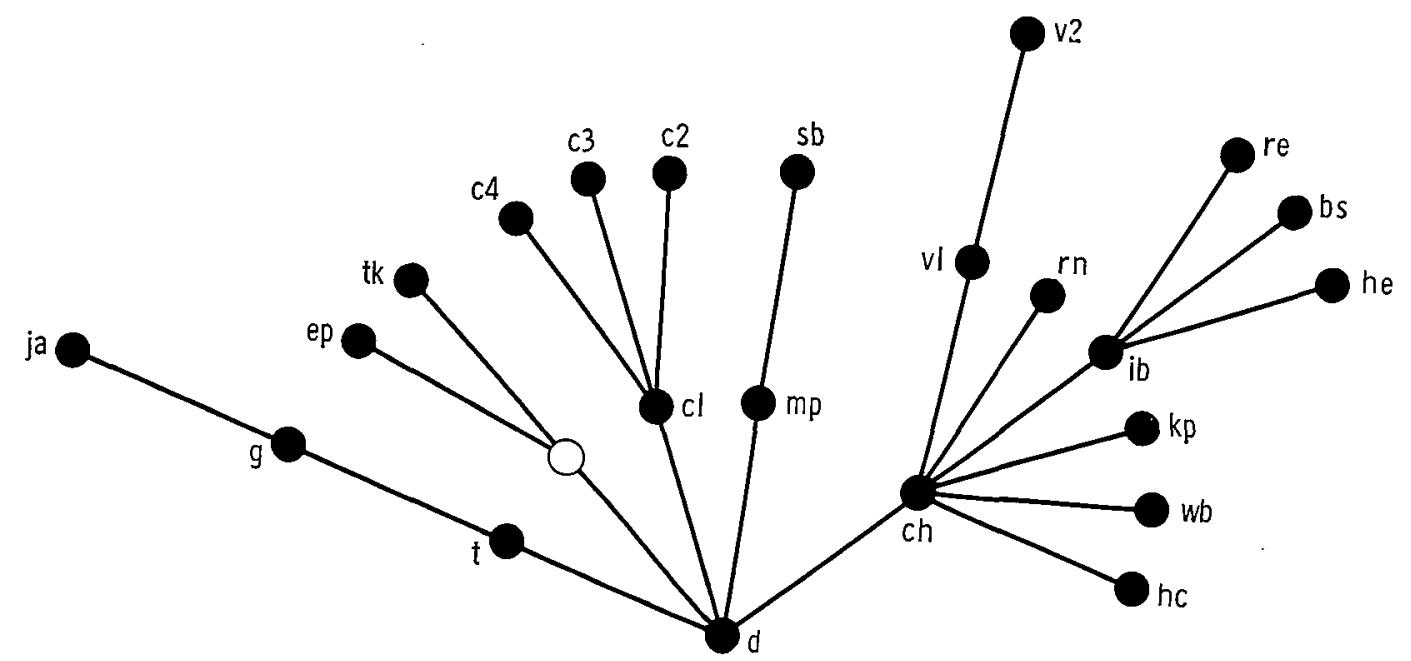

FIG. 33. An estimate of the phylogeny of the Charadrii. Abbreviations: bs, Cladorhynchus; c1, Rhinoptilus (part), Glareola; c2, Cursorius; c3, Rhinoptilus cinctus; c4, Stiltia; ch, Peltohyas, Charadriinae (part); d, Dromas; ep, Pluviantus; g, Stercorariidae (part), Larinae; hc, Hoploxypterus; he, Haematopus; ib, Ibidorhyncha, Himantopus; ja, Stercorariidae (part); kp, Charadrius pecuarius; mp, Pluvianellus; re, Recurvirostra; rn, Charadriinae (part); sb, Chionis; t, Sterninae; tk, Burhinidae; v1, Vanellinae (part); v2, Vanellinae (part); wb, Anarhynchus.

On the tree defined by the pair of cliques which include Characters 13 and 19 (see Fig. 32) five phyletic lines radiate from the ancestor of this assemblage. The Rostratulidae (node ro) share derived states of Characters 8 and 35; the Phalaropodidae (node ph) share a derived state of Character 35; Tringa, Catoptrophorus, and Heteroscelus (nodes t1a, t1b, and t2) share derived states of Characters 8, 17, and 22; Prosobonia, Bartramia, and Numenius (node cu) share a derived state of Character 13; and the sandpipers, snipes, and woodcocks (nodes bb, do, go, pe, s1, s2, s3, sa, tr, ts, w1, w2, and xe) share a derived state of Character 4.

Since they contain too few species, the Rostratulidae and Phalaropodidae were not analysed further. The other lines were examined in greater detail (Strauch, 1976). The results of those analyses and subsequent work on the Scolopaci will be reported elsewhere. 


\section{The Charadrii}

The compatibility analysis of the 56 characters which vary among the 137 EUs in this suborder yielded a largest clique of 22 characters:

Charadrii Clique: $1,3,6,7,10,11,12,15,33,37,39,41,45,46,49,53,54,55,58$, $59,66$.

On a tree defined by this clique (Fig. 33) Dromas (node d) is not separated from the ancestor of the suborder. (An analysis to define better the relationship of Dromas to the rest of the suborder is discussed below.) This tree defines five phyletic lines in the Charadrii. The Stercorariidae and Laridae (nodes $\mathrm{g}$, ja and t) share derived states of Characters 37, 41, and 49; Pluvianus and the Burhinidae (nodes ep and tk) share derived states of Characters 1 and 7; the Glareolidae, except Pluvianus and Peltohyas (nodes c1, c2, c3, and $\mathrm{c} 4$ ), share derived states of Characters 3 and $11 ;$ Pluvianellus and Chionis (nodes mp and sb) share derived states of Characters 21 and 46; the species of Chionis (node sb)

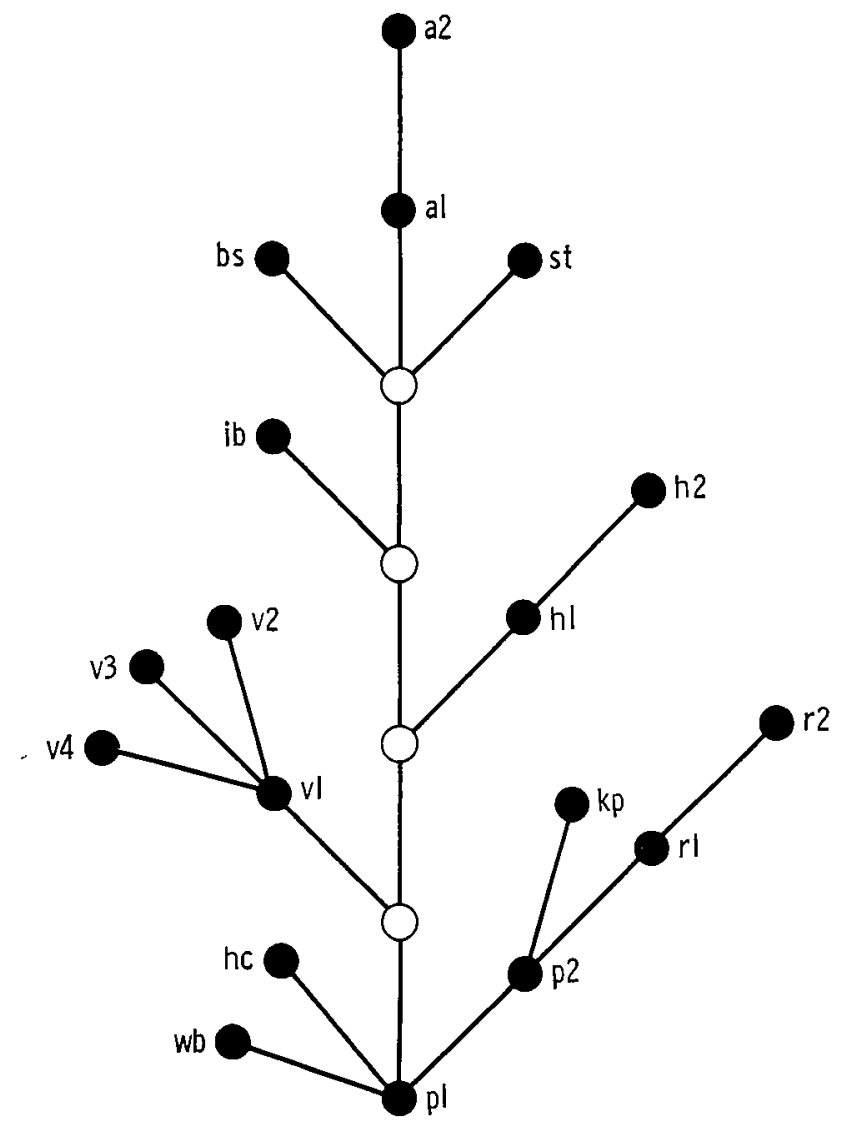

FIG. 34. An estimate of the phylogeny of the plovers and their allies. Abbreviations: a1, Recurvirostra (part); a2, R. avosetta; bs, Cladorhynchus; h1, Haematopus (part); h2, Haematopus (part); hc, Hoploxypterus; ib, Ibidorhyncha; kp, Charadrius pecuarius; p1, Peltohyas, Pluvialis, Charadrius (part), Phegornis; p2, Charadrius (part). Thinornis; r1, Charadrius (part); r2, C. placidus; st, Himantopus; v1, Vanellus (part); v2, Vanellus (part); v3, V. lugubris; v4. V. tricolor; wb, Anarhynchus. 
share a derived state of Character 53, while the plovers and their allies (nodes bs, ch, hc, he, ib, kp, re, rn, v1, v2 and wb) share derived states of Characters 10 and 21.

This analysis indicates that neither Pluvianus nor Peltohyas is a member of the Glareolidae, that Peltohyas is a member of the Charadriidae, and that Pluvianellus is not a member of the Charadriidae but rather is close to Chionis. Since there are so few species in Pluvianellus and Chionis, no further analysis was done on the phyletic line leading to them. Each of the monophyletic lines identified in Fig. 33 was analysed in greater detail (Strauch, 1976). The analyses of the line leading to the Stercorariidae and Laridae, that leading to Pluvianus and the Burhinidae, and that leading to the Glareolidae will be discussed in greater detail elsewhere. The preliminary analysis of the line leading to the plovers and their allies follows.

The plovers and their allies. The compatibility analysis of the 35 characters which vary among the 78 EUs in this group yielded one largest clique of 21 characters:

Plovers and Allies Clique: 2, 6, 8, 12, 15, 20, 21, 25, 27, 29, 33, 38, 41, 46, 47, 50, 51, $59,60,64,67$.

The tree defined by this clique (Fig. 34) shows the small plovers, Charadriinae (nodes hc, $\mathrm{kp}, \mathrm{p} 1, \mathrm{p} 2, \mathrm{r} 1, \mathrm{r} 2$, and $\mathrm{wb}$ ) as the basal group of this line. The lapwings (nodes v1, v2, v3), oystercatchers (nodes h1, h2), the Ibisbill (node ib), and the avocets and stilts (nodes a1, a2, bs, and st) share a derived state of Character 29 .

\section{Analysis of the Charadrii using hypothetical ancestors}

My original analysis of the Charadrii produced a tree (Fig. 33) on which Dromas is not separated from the ancestor of the suborder. Furthermore, the other lines in the

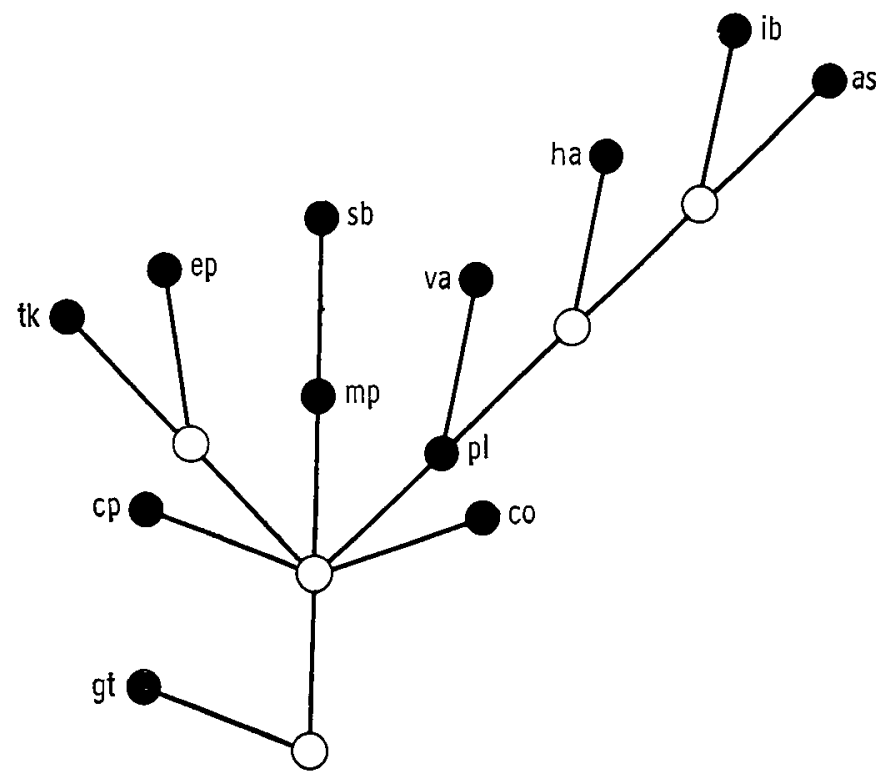

FIG. 35. An estimate of the phylogeny of the Charadrii using hypothetical ancestors. Abbreviations: as, hypothetical ancestor of the avocets and stilts; co, hypothetical ancestor of the Glareolidae; cp, Dromas; gt, hypothetical ancestor of the Stercorariidae and Laridae; ha, hypothetical ancestor of Haematopus; ib, Ibidorhyncha; mp, Pluvianellus; p1, hypothetical ancestor of the Charadriinae; sb, Chionis; va, hypothetical ancestor of the Vanellinae. 
suborder radiate fanwise from the ancestor, a result which indicates little about the relationships among the various lines. I then investigated the possibility that some of the characters rejected in this analysis would prove compatible with the original clique if some of the lines were replaced by their hypothetical ancestors. Hypothetical ancestors were constructed for the Stercorariidae and Laridae, Glareolidae, Charadriinae, Vanellinae, Haematopus, and the avocets and stilts. The character states for the ancestors were estimated as follows: the ancestor was assigned the most primitive state found in the group for all characters included in the largest clique in the analysis of the group; for characters rejected in the analysis of the group the ancestor was assigned the state found in the majority of the EUs placed near the base of the group tree; if the ancestral state for the group could not be assigned by these two methods, the most primitive state found in the group was used. The hypothetical ancestors constructed thus were used along with the real EUs Dromas, Pluvianus, Pluvianellus, Chionis, Peltohyas, and Ibidorhyncha for a compatibility analysis. The compatibility analysis of the 42 characters which vary among the 13 real and hypothetical EUs in this group yielded one largest clique of 26 characters:

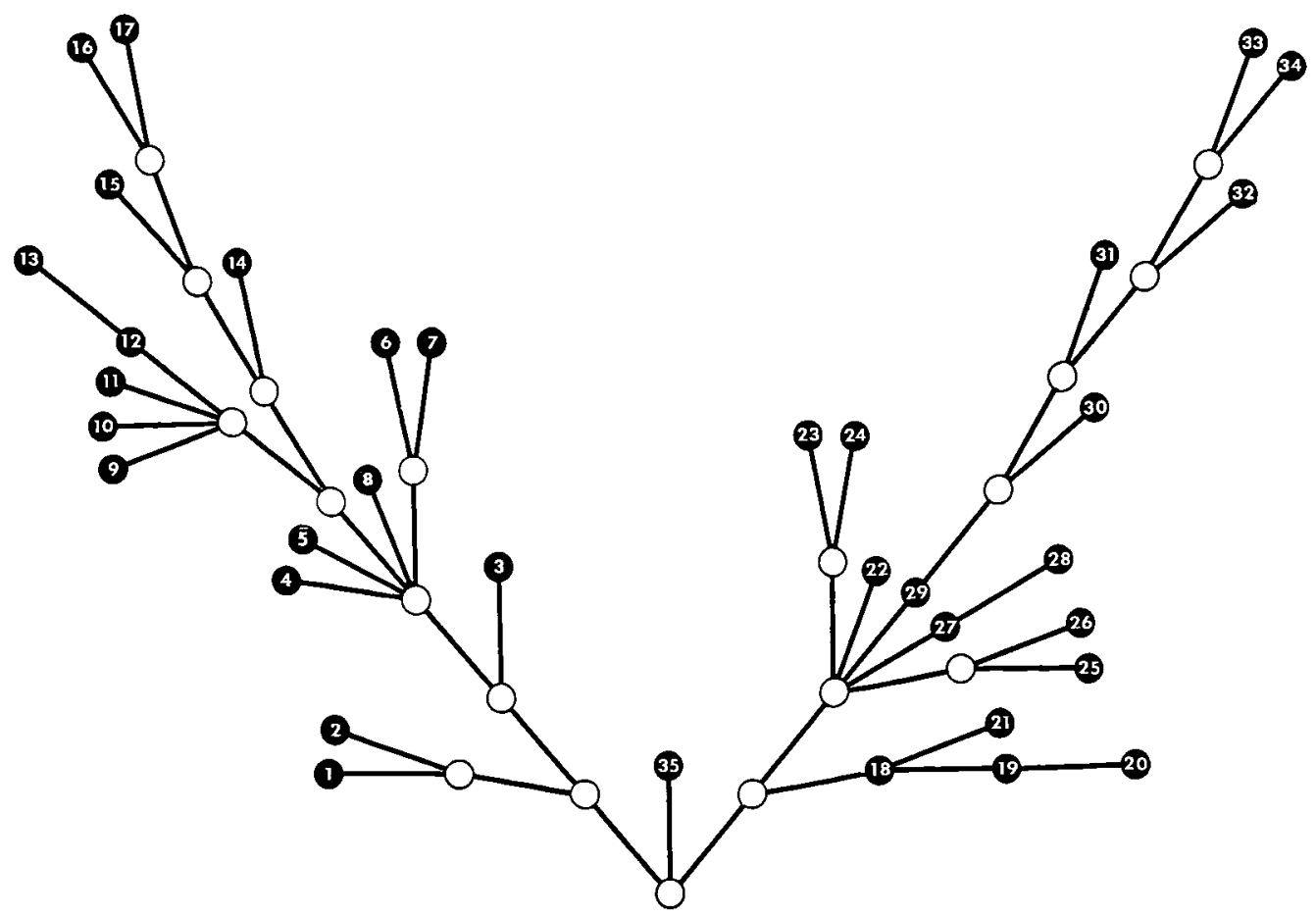

Frg. 36. Final estimate of the phylogeny of the Charadriiformes. Node 1: Microparra, Actophilornis, Irediparra, Metopidius; 2: Hydrophasianus, Jacana; 3: Thinocoridae; 4: Rostratulidae; 5: Phalaropodidae; 6: Prosobonia; 7: Bartramia, Numenius; 8: Tringa, Catoptrophorus, Heteroscelus; 9: Actitis, Aphriza, Calidris, Eurynorhynchus, Micropalama, Tryngites, Philomachus, Limicola; 10: Arenaria; 11: Limnodromus; 12: Xenus; 13: Limosa; 14: Coenocorypha; 15: Lymnocryptes; 16: Gallinago; 17: Philohela, Scolopax; 18: Sterninae; 19: Larinae: 20: Stercorariidae; 21: Rynchops; 22: Dromas; 23: Burhinidae; 24: Pluvianus; 25: Cursoriinae; 26: Glareolinae; 27: Pluvianellus; 28: Chionis; 29: Charadriinae, Peltohyas; 30: Vanellinae; 31: Haematopus; 32: Ibidorhyncha; 33: Himantopus; 34: Cladorhynchus, Recurvirostra; 35: Alcidae. 
Charadrii Clique B: $1,3,7,10,11,12,16,20,21,32,33,34,37,38,41,44,45,46$, $49,51,53,57,58,68,69$.

Characters $6,15,39,54,55,59$, and 66 , which were included in the original Charadrii clique, were not included in this analysis because they are constant throughout the real and hypothetical EUs used in this analysis. Characters 16, 20, 23, 32, 34, 38, 44, 51, 57 , 68 , and 69 , which were rejected in the original analysis, are included in the largest clique found in this analysis. The tree defined by Charadrii Clique B (Fig. 35) has five internodes not on the original Charadrii tree (Fig. 33); these are defined by Characters 20, 23, 44, 57, 68, and 69. The steps defined by Characters 20,57, 68, and 69 are found in further analyses of Haematopus, Ibidorhyncha, Himantopus, Cladorhynchus, and Recurvirostra discussed elsewhere (Strauch, 1976). The steps defined by Characters 23 and 44, however, are unique to this analysis. All the Charadrii except the Stercorariidae and Laridae (nodes as, co, cp, ep, ha, ib, mp, pl, sb, tk, and va) share the derived state of Character 23 and thus form a sister group with the Stercorariidae and Laridae (node gt). The derived state for Character 23 has appeared several times in the Stercorariidae and Laridae. Dromas (node $\mathrm{cp}$ ) is on a line defined by the derived state of Character 44 in this analysis. The derived state of this character occurs in both the Stercorariidae and Laridae and the Glareolidae.

\section{Summary of the cladistic relationships among the Charadrifformes}

Figure 36 shows the final estimate of the phylogenetic history of the Charadriiformes based on the characters described herein. This tree, a composite of the analyses discussed above and others reported elsewhere (Strauch, 1976), was constructed by replacing each branch in the first charadriiform tree (Fig. 32) with the branch found in the analysis of that branch alone. This procedure was repeated until most of the cladistic detail found in the analyses had been placed on the tree. For clarity some of the detail found in the analyses of smaller groups has been omitted (e.g. the Charadriinae are represented only by node 29). The character states which define each monophyletic group in Fig. 36 are identified in Table II.

TABLE II

Character states defining monophyletic groups in Fig. 36

\begin{tabular}{lll}
\hline \multicolumn{2}{c}{ Nodes } & $\begin{array}{c}\text { Character } \\
\text { number }\end{array}$ \\
\hline $1-17$ & 14 & maxillopalatine strut A absent \\
& 39 & coracoidal foramen absent \\
& 42 & ridge in capital groove of humerus \\
$1-2$ & 44 & poorly-developed pneumatic fossa II of humerus \\
& 45 & ectepicondylar prominence of humerus absent \\
& 46 & ancestral Jacanidae state of extensor process of metacarpus of carpometacarpus \\
& 47 & intermetacarpal tuberosity lying within groove formed at junction of metacarpals II and III \\
& 48 & ossified bridge over distal metacarpal symphysis of carpometacarpus \\
& 52 & lateral synsacral strut falling short of acetabulum \\
1 & 61 & trochlea for digits II and IV only slightly elevated compared to that for digit III \\
2 & 46 & jacana wing knobs and blade-like radius \\
$3-17$ & 46 & jacana wing spurs \\
& 24 & origin of M. complexus on vertebra 3 absent \\
\hline
\end{tabular}


TABLE II-Continued

\begin{tabular}{|c|c|c|}
\hline Nodes & $\begin{array}{l}\text { Character } \\
\text { number }\end{array}$ & Shared derived character state \\
\hline & 61 & $\begin{array}{l}\text { trochlea for digits II and IV considerably elevated compared to that for digit III, trochlea for } \\
\text { digit III more so than that for digit IV }\end{array}$ \\
\hline \multirow[t]{2}{*}{3} & 4 & angle between lateral nasal bar and jugal bar about 70 degrees or more \\
\hline & 8 & short, stubby, finch-like bill tip \\
\hline $4-17$ & 10 & basipterygoid articulation of pterygoid and basipterygoid processes on basisphenoid rostrum \\
\hline 4 & 8 & dorsal bar of upper jaw flattened with a distinctive ridge along top \\
\hline 5 & 8 & distinct flange of bone on dorsal bar of upper jaw \\
\hline $6-7$ & 13 & flat sheet of bone at junction of jugal bar and ventral bar of upper jaw \\
\hline \multirow[t]{2}{*}{6} & 51 & posterior synsacral strut present \\
\hline & 57 & posterior medial and medial edge of ilium forming smooth curve \\
\hline \multirow[t]{2}{*}{7} & 12 & maxillopalatines and palatines considerably but not completely fused \\
\hline & 29 & origin of $M$. splenius capitis on vertebrae 2 and 3 \\
\hline \multirow[t]{3}{*}{8} & 8 & dorsal and ventral bars of upper jaw fused for about one-half length of bill \\
\hline & 17 & maxillopalatine strut $\mathrm{D}$ present \\
\hline & 22 & posterior process of mandible normal with corresponding bump on supraangular present \\
\hline $9-17$ & 4 & angle between lateral nasal bar and jugal bar about 70 degrees or more \\
\hline $9-13$ & 5 & zygomatic process shorter than suprameatic process \\
\hline 9 & 19 & foramen magnum distinctly heart-shaped \\
\hline 10 & 8 & bill tip a sharp, short cone \\
\hline 11 & 9 & cross-section of dorsal bar of upper jaw T-shaped \\
\hline $12-13$ & 64 & tendinal canal No. 1 of hypotarsus a groove \\
\hline \multirow[t]{3}{*}{13} & 9 & cross-section of dorsal bar of upper jaw like inverted $U$ \\
\hline & 12 & maxillopalatines and palatines considerably but not completely fused \\
\hline & 60 & $\begin{array}{l}\text { junction of anterior and posterior crests of pelvis forming distinct projection over ilioischiatic } \\
\text { fenestra }\end{array}$ \\
\hline \multirow[t]{4}{*}{$14-17$} & 3 & ectethmoid and frontal fused \\
\hline & 20 & exorbital nasal gland \\
\hline & 21 & prearticular process of mandible expanded and hollow and without flange \\
\hline & 22 & posterior process of mandible shifted posteriad \\
\hline 14 & 50 & synsacral strut extending from fourth vertebra \\
\hline \multirow[t]{5}{*}{$15-17$} & 1 & lacrimal-ectethmoid complex connected with postorbital process \\
\hline & 5 & zygomatic process connected with lacrimal-ectethmoid complex \\
\hline & 15 & maxillopalatine strut $B$ absent \\
\hline & 59 & ischium and pubis fused immediately posterior to obturator foramen \\
\hline & 70 & tendinal canals of hypotarsus for tendons No's 2,3 , and 4 lying in straight line \\
\hline 15 & 36 & medial sternal notch present \\
\hline \multirow[t]{2}{*}{$16-17$} & 25 & no origin of M. complexus on vertebra 4 \\
\hline & 54 & posterior end of renal depression deep and abrupt \\
\hline 16 & 66 & tendinal canal of hypotarsus for tendon No. 3 a bony canal \\
\hline \multirow[t]{3}{*}{17} & 20 & nasal gland neither supraorbital nor exorbital \\
\hline & 23 & posterior mandibular fossa absent \\
\hline & 58 & ischial angle relatively short and about even with posterior projection of ilium \\
\hline $18-34$ & 43 & anconal surface of deltoid crest of humerus concave \\
\hline \multirow[t]{3}{*}{$18-21$} & 37 & posterior lateral sternal process and xiphial area extending about equal distance posteriad \\
\hline & 41 & distinct groove extending into furcular process \\
\hline & 49 & proximal phalanx, digit III, perforated \\
\hline \multirow[t]{2}{*}{$19-20$} & 54 & distinct strut at posterior end of renal depression \\
\hline & 57 & posterior medial and medial edge of ilium forming smooth curve \\
\hline 20 & 67 & no canal for tendon No. 4 on hypotarsus \\
\hline
\end{tabular}


TABLE II-CContinued

\begin{tabular}{|c|c|c|}
\hline Nodes & $\begin{array}{l}\text { Character } \\
\text { number }\end{array}$ & Shared derived character state \\
\hline 21 & 32 & 12 neck vertebrae \\
\hline $22-34$ & 23 & posterior mandibular fossa absent \\
\hline 22 & 44 & pneumatic fossa II of humerus poorly developed \\
\hline \multirow[t]{2}{*}{$23-24$} & 1 & lacrimal-ectethmoid complex abutting jugal bar \\
\hline & 7 & holorhinal nostrils \\
\hline \multirow{5}{*}{23} & 30 & origins of M. rectus capitis only on vertebrae $1-4$ \\
\hline & 33 & burhinid cervical vertebral strut present \\
\hline & 45 & no ectepicondylar prominence of humerus \\
\hline & 58 & ischial angle relatively short and about even with posterior projection of ilium \\
\hline & 63 & angle formed by trochleae for digits II and IV smaller than 25 degrees \\
\hline \multirow[t]{4}{*}{24} & 24 & no origin of $\mathrm{M}$. complexus on vertebra 3 \\
\hline & 29 & origin of $M$. splenius capitis on vertebrae 2 and 3 \\
\hline & 46 & wing knob present \\
\hline & 63 & angle formed by trochleae for digits II and IV larger than 35 degrees \\
\hline \multirow[t]{2}{*}{$25-26$} & 3 & ectethmoid and frontal fused \\
\hline & 11 & medial condyle of quadrate raised and twisted so that it faces outward \\
\hline \multirow[t]{2}{*}{25} & 44 & pneumatic fossa II of humerus poorly developed \\
\hline & 62 & hallux absent \\
\hline 26 & 30 & origins of $M$. rectus capitis only on vertebrae $1-4$ \\
\hline \multirow[t]{2}{*}{$27-28$} & 21 & no flange on prearticular process of mandible \\
\hline & 46 & wing knobs present \\
\hline 28 & 53 & four parapophyses on lumbar vertebrae \\
\hline \multirow[t]{2}{*}{$29-34$} & 10 & basipterygoid articulation of pterygoid and basipterygoid processes on basisphenoid rostrum \\
\hline & 21 & flange on prearticular process of mandible fused to supraangular \\
\hline $30-34$ & 29 & origins of M. spleniuscapitis on vertebrae 2 and 3 \\
\hline 30 & 33 & Vanellinae cervical vertebral strut present \\
\hline \multirow[t]{2}{*}{$31-34$} & 12 & maxillopalatines and palatines considerably but not completely fused \\
\hline & 64 & tendinal canal No. 1 of hypotarsus a groove \\
\hline \multirow[t]{4}{*}{31} & 4 & angle between lateral nasal bar and jugal bar about 70 degrees or more \\
\hline & 12 & maxillopalatines and palatines completely fused \\
\hline & 32 & 12 neck vertebrae \\
\hline & 38 & ventral manubrial spine of sternum not dividing coracoidal sulci \\
\hline \multirow[t]{2}{*}{$32-34$} & 27 & no origin of M. complexus on vertebra 6 \\
\hline & 57 & posterior medial and medial edge of ilium forming smooth curve \\
\hline \multirow[t]{3}{*}{32} & 2 & lacrimal and ectethmoid not in contact \\
\hline & 20 & exorbital nasal gland \\
\hline & 30 & origins of $M$. rectus capitis only on vertebrae $1-4$ \\
\hline \multirow[t]{4}{*}{$33-34$} & 8 & bill tip long, dorsal and ventral bars of upper jaw fused for about one-half length of bill \\
\hline & 61 & $\begin{array}{l}\text { trochlea for digits II and IV considerably elevated compared to that for digit III, trochlea for } \\
\text { digit II more so than that for digit IV }\end{array}$ \\
\hline & 63 & angle between trochleae for digits II and IV larger than 35 degrees \\
\hline & 68 & tendinal canal No. 6 of hypotarsus present as a groove \\
\hline $33-34$ & 69 & tendinal canal No. 5 of hypotarsus present as a groove \\
\hline \multirow[t]{2}{*}{33} & 35 & five costal processes on sternum \\
\hline & 60 & $\begin{array}{l}\text { junction of anterior and posterior crests of pelvis forming distinct projection over ilioischiatic } \\
\text { fenestra }\end{array}$ \\
\hline 34 & 65 & tendinal canal No. 1 of hypotarsus directly anterior to tendinal canal No. 2 (reversal) \\
\hline \multirow[t]{2}{*}{35} & 40 & brachial tuberosity of coracoid twisted so that it does not roof triosseal canal \\
\hline & 52 & no lateral synsacral strut \\
\hline
\end{tabular}




\section{Discussion}

The initial choice of the members of a study collection is critical to any study. To the extent that the membership of the study collection deviates from a true monophyletic assemblage, any estimate of phylogenetic relationships within the group will be distorted. Usually some members of a monophyletic group are unavailable for inclusion in the study collection. If the missing members are closely related to some of the members in the collection and differ little from them, their absence will have little effect on the outcome of the analysis. If, however, the missing members represent distinct lineages not otherwise represented in the study collection, omitting them can seriously affect the cutcome of any analysis. When such groups are omitted, distinctive elements of the diversity of the group, such as unique character states and unique combinations of character states, are neglected in the analysis.

Unfortunately, most workers considered only a portion of the known diversity among the Charadriiformes when attempting to determine their relationships. The idea that the order consists of three distinct and presumably monophyletic groups: the waders, the Lari, and the alcids, dates from Gadow's (1892) classification. That the Lari and the alcids are monophyletic assemblages is almost universally accepted. Agreement on the status of the waders, however, is not universal. Previous workers either assumed that all waders represent a monophyletic group (Jehl, 1968; Burton, 1974), or they assumed that the Charadriidae and Scolopacidae together form a monophyletic group and then placed the remaining waders with them or with the Lari (Shufeldt, 1903; Lowe, 1931b; Yudin, 1965). Even Lowe (1916a), who thought that a "gull is only a highly specialized plover", placed waders and the Lari in different suborders. The results of this study indicate that it is unlikely that the Charadriidae and Scolopacidae together form a monophyletic group within the order. Jehl (1968) noted the distinctiveness of the plovers and the sandpipers, but he argued only that they represent distinct families. Only Ahlquist (1974) found that the plovers are closer to the Lari than to the sandpipers.

All of the known diverse elements of the Charadriiformes were included in this study. Skeletons of almost all species of waders, all but one of Peters' (1934) genera of Lari, and species representing the major lines of the alcids (Storer, 1945) were studied (see Table I). My analyses have thus not neglected any of the major diversity within the order. The persuasiveness of all the characters used in defining my groups is not discussed in detail below; it lies in their inclusion in the cliques, in which the characters together support a consistent story.

\section{The Charadriiformes}

My division of the order into three phyletic lines (Fig. 36) differs from past work in dividing the waders into two major groups, the scolopacine and charadriine waders sensu lato, in placing the charadriine waders on a line with the Lari, and in not placing the Lari and alcids on the same line.

In spite of Bock's (1958) view that "There is no longer any need for maintaining family status for the plovers ... the most consistent classification is to include the plovers as a subfamily of an enlarged family of shorebirds", there is abundant evidence that the plovers and sandpipers are very different. Osteologically they differ in five of the primary characters found in this study, as well as in several others described herein and in Lowe $(1915 b, 1925$, 
1926, 1931b) and Zusi \& Jehl (1970). Sibley \& Ahlquist (1972) and Ahlquist (1974) discuss the differences in the electrophoretic patterns of their egg-white proteins. Jehl (1968) points out the differences in the plumage patterns of their downy young. Burton (1974) found several consistent differences in feeding behaviour and in jaw musculature and tongue structure. Although few species have yet been examined, the sperm morphology of the Scolopacidae is unique among non-passerine birds, whereas sperm of the Charadriidae, Laridae and Alcidae are almost identical and similar to that of other non-passerine orders (Retzius, 1909, 1911; McFarlane, 1963).

Gadow (1892) characterized his Charadriidae, which include both plovers and sandpipers, as cosmopolitan, schizorhinal and having basipterygoid processes. But the Scolopacidae and Charadriidae have very different geographic distributions; all charadriiform birds except Pluvianus and the Burhinidae are schizorhinal; and, as shown in this study, the condition of the basipterygoid processes is probably not a true cladistic character at the ordinal level. Lowe's $(1931 b)$ table of 14 characteristics which distinguish the suborders of his order Telmatomorphae includes only one, the presence of basipterygoid processes, which is unique to his suborder Limicolae (Rostratulidae, Charadriidae, and Scolopacidae).

Showing that the Charadriidae and Scolopacidae represent two distinct phyletic lines, however, does not in itself demonstrate that they are not a monophyletic line sharing a common ancestor not shared by any other charadriiform. It is only necessary to support the contention, as has been done here, that each shares most recent common ancestors with other groups which are not shared with each other. As discussed above, few workers have included non-waders in their considerations when exploring these relationships.

A close relationship between the alcids and Lari has been postulated many times (Gadow, 1892; Boetticher, 1934; Storer, 1960; Yudin, 1965; Sibley \& Ahlquist, 1972; Ahlquist, 1974). Hudson, Hoff et al. (1969), however, argued that the two groups differed sufficiently in their wing and leg musculature to be placed in separate suborders. None of the characters used in this study indicate that the Lari and alcids form a monophyletic assemblage which excludes other charadriiforms. (Hudson, Hoff et al. (1969) report that they share a patagial fan sesamoid not found in the Charadrii they examined.) They do, however, share the primitive states of many characters and in that sense are similar. Possibly my Charadrii and the Alcae together represent a monophyletic line which does not include the Scolopaci, but I have no evidence for this view. The alcids are thus best considered distinct from the Lari unless new evidence indicates otherwise.

\section{The suborders of the Charadriiformes}

\section{The Scolopaci}

The Jacanidae, Rostratulidae, Thinocoridae, Phalaropodidae, and Scolopacidae are a monophyletic group (Fig. 30) defined by absence of a maxillopalatine strut A (Fig. 13), absence of a coracoidal foramen (Fig. 21), and presence of a ridge in the capital groove of the humerus (Fig. 23).

The relationships of the Jacanidae, Rostratulidae, and Thinocoridae have been the subject of considerable debate. Forbes (1881) argued on the basis of pterylosis, development of the cecum, morphology of the patagial tendons, and osteology that jacanas are more like waders than they are like rails, and, indeed, Gadow's (1892) and most later classifications included them in the Charadriiformes. Lowe (1925), on the basis of the 
differences in the quadrato-tympanic articulation observed between Gruiformes and Charadriiformes, argued that jacanas are rails. He also declared that the colour pattern of downy young is crane-like, even though jacana young are strongly patterned (Jehl, 1968) and crane young hardly at all (Walkinshaw, 1973; pers. obs.); that the possession of basipterygoid processes places them with the more primitive Gruiformes, even though the processes are rare in the Gruiformes but are the usual condition in the Charadriiformes; and that jacanas have a ralline pelvis when compared with plovers, although comparison with the pelvis of a painted snipe or a true snipe shows the resemblance to rails to be superficial. In the same paper Lowe pointed out that the quadrato-tympanic articulation of the Scolopacidae is also somewhat gruiform, which he attributed to the close relationship of the Gruiformes and Charadriiformes. Later he decided (Lowe, 1931a) that jacanas are not really rails but "apparently a more generalized or primitive group than the rest of the Gruiformes". This appears only to sidestep the issue of their true affinities. Except for Verheyen (1967), who based his classification on an idiosyncratic type of numerical taxonomy, and Stresemann (1959), who placed every distinctive group in a separate order, all recent workers place the Jacanidae in the Charadriiformes. Kitto \& Wilson (1966) found the mobility of their S-malate dehydrogenase like that of other charadriiforms.

Many recent workers have suggested that the jacanas are closest to the painted snipes. Yudin (1965) thought that Jacana represents an early radiation of the waders which took place before the plovers and sandpipers diverged, apparently because he assumed that Pluvialis represents the primitive charadriiform type and found that Jacana and Pluvialis differ little in their jaw musculature. Ahlquist (1974) found that the IFPA patterns of the egg-white proteins of the jacanas resemble those of the sandpipers.

My analyses indicate that the Jacanidae are a monophyletic group defined by a poorlydeveloped pneumatic fossa II of the humerus (Fig. 23), lack of an ectepicondylar prominence on the humerus (Fig. 23), a specialized condition of the extensor process of the metacarpus (see Character Descriptions for the hypothetical ancestral jacana state used here), an intermetacarpal tuberosity lying within the groove formed by the junction of metacarpals II and III, an ossified bridge over the distal metacarpal symphysis of the carpometacarpus (Fig. 24), a lateral synsacral strut which falls short of the acetabulum (Fig. 26), and the trochlea for digits II and IV only slightly elevated compared to that for digit III (Fig. 28). The remainder of the scolopacine waders are a monophyletic group defined by an origin of M. complexus on vertebra 3 and the trochlea for digits II and III considerably elevated compared to that for digit III, and with the trochlea for digit II more raised than that for digit IV (Fig. 28). The jacanas may also be characterised by their usually dark glossy plumage, often naked or wattled forehead, long toes and claws, and by their ecology.

The painted snipes (Rostratulidae) are usually thought to be closest to the jacanas (Beddard, 1901; Jehl, 1968; Sibley \& Ahlquist, 1972; Ahlquist, 1974) or to the Scolopacidae, perhaps near the true snipes (Yudin, 1965; Ahlquist, 1974). Lowe (1931a) considered them waders but distinctive enough to be given family rank. In my analyses they cluster with the Scolopacidae (Fig. 32) because they possess basipterygoid processes; they are distinguished by their unique bill morphology (Fig. 8).

The Thinocoridae superficially resemble grouse or finches, probably reflecting adaptations for their (atypical for Charadriiformes) vegetarian diet. Since Gadow (1892) they have usually been accepted as modified Charadriiformes of uncertain relationship. Garrod (1877) thought them closest to the Glareolidae on the basis of palate structure and 
lack of basipterygoid processes. Lowe (1931a) first placed the Thinocoridae in a suborder with the Glareolidae, Chionididae, Dromadidae, and Laridae, but later (1931 $b$ ) placed them in a suborder of their own. Boetticher (1934) thought the Thinocoridae closest to Chionis because to him they both seemed to be "primitive collective types". On similar grounds, as well as because of similarities in jaw musculature, basic similarity of body structure, presence of a crop, and lack of toe webbing, Yudin (1965) also thought the Thinocoridae closest to Chionis. Jehl (1968) concluded that the downy plumage of seedsnipes offered no clue to their relationships and followed Yudin in placing them near the Chionididae.

I find the seedsnipes to be a sister group to the Scolopacidae (Fig. 32), defined by the angle between the lateral nasal bar and the jugal bar of more than $70^{\circ}$ and by a short, stubby, finch-like bill tip (Fig. 8). I did not work out the relationships among them. Lowe $(1931 b)$ found the Thinocoridae like the sandpipers in downy plumage pattern, pterylosis, patagial fan, and several osteological characters. Since he also noted that several features of the skull seemed gruiform, he placed them in a separate suborder between waders and his Grues. Ahlquist (1974) presented protein evidence that there is a "real connection among the seedsnipes, painted snipes, and sandpipers" but failed to define its exact form.

The data and analyses presented here strongly support the hypothesis that the Jacanidae, Rostratulidae, Thinocoridae, and Scolopacidae are a monophyletic group. The cladistic relationships found among these four groups are not as strongly supported and require further study. While it is clear that the jacanas and sandpipers represent the two major phyletic lines of scolopacine waders, the problem is to determine the positions of the painted snipes and seedsnipes. The skeletons of the painted snipes and the jacanas seem similar in many ways, but this is not expressed in the compatibility analyses. Perhaps, as Ahlquist (1974) suggests, there is a "real distant relationship between the jacanas and the painted snipes". On the other hand, the painted snipes are similar to the true snipes in some skeletal features, but my results indicate that these represent convergence rather than recent common ancestry.

The Thinocoridae may be even closer to the Scolopacidae than my analyses indicate. Besides the similarities of their downy young (discounted by Jehl, 1968), the pattern of the head and upper back plumage of juvenile and some adults is similar to that of sandpipers.

The sperm morphology of the Jacanidae, Rostratulidae, and Thinocoridae is unknown. In light of the distinctive sperm morphology of the Scolopacidae, such knowledge might be valuable in unraveling the relationships among these four families.

\section{The Charadrii}

My analyses yield a monophyletic line which includes the Stercorariidae, Laridae, Rynchopidae, Dromadidae, Burhinidae, Glareolidae, Chionididae, Charadriidae, Haematopodidae, and Recurvirostridae (Fig. 30), defined by a concave anconal surface of the deltoid crest (Fig. 23). Since most authors have not considered the Lari when studying the relationships among the waders, little evidence has been published in support of this arrangement. Burton (1974, pers. comm.), in studies restricted to waders, found that only the Dromadidae, Burhinidae, Pluvianus, Chionis, Pluvianellus, Peltohyas, the Charadriidae and Recurvirostridae possess the Ap. $\mathrm{A}^{1}$ slip of $\mathrm{M}$. adductor externus, while the Haematopodidae do not. He also found that $N$. pterygoideus passes through Part $\mathrm{O}$ of $\mathbf{M}$. 
pterygoideus only in the Burhinidae, Pluvianus, Chionis, Pluvianellus, the Charadriidae, Haematopodidae, and Recurvirostridae. In a specimen of Larus argentatus N. pterygoideus passes between Parts $\mathrm{O}$ and $\mathrm{M}$ of M. pterygoideus (Orenstein, pers. comm.) as it does in Dromas, Rhinoptilus, Cursorius, Stiltia, and Glareola (Burton, pers. comm.). These two characters tend to confirm some of my findings of relationship; however, the condition in the remaining genera will have to be ascertained before more definite conclusions can be drawn.

The Charadrii consist of two major lines (Fig. 33): one leading to the skuas, gulls, terns, and skimmers, which is defined by the posterior lateral sternal processes and the extension of the xiphial area extending about equally posteriad, extension of a groove into the furcular process (Fig. 22), and a perforated proximal phalanx, digit III; and another leading to the charadriine waders sensu lato, defined by lack of a posterior mandibular fossa (Fig. 17).

The relationships of the Crab plover, Dromas ardeola, which looks like a combination of gull, thick-knee, and avocet, have long been a puzzle. Van der Hoeven (1868) pointed out skeletal similarities with both thick-knees and oystercatchers. Lowe (1916b) noted that its pterylosis differs sharply from that of plovers and sandpipers, that the morphology of the occipital and squamosal regions, as well as the squamosal articulation of the quadrate, are gull-like, that the lacrimal has a modified plover-like arrangement, that the palatal structures are like those of the thick-knees, and that the humerus is plover-like. He decided that its affinity with these other groups was one of "a common ordinal or subordinal fellowship". Mainly on the basis of the lack of basipterygoid processes in the adult he later (Lowe, 1931b) placed it with the Glareolidae and Chionididae in his suborder Lari-Limicolae. Boetticher (1934) thought Dromas belonged between the coursers and thick-knees. Yudin (1965) also discussed the resemblance of the skull of Dromas to those of the Lari; he thought that "the similarity to terns shows a convergent character, to gulls consanguinity". He concluded that Dromas represents an early offshoot of a line which also gave rise to the Lari. Jehl (1968) found that downy plumage of Dromas to give no clue to relationships and suggested that the "similarity of the adult in body form to burhinids and in plumage pattern to the recurvirostrids might indicate they are distantly related to that major line".

Dromas was not distinguished from the ancestor of the suborder by any of the primary characters in the original analysis of my Charadrii (Fig. 33). While Dromas shares few derived character states with any of the other Charadrii, it shares many primitive character states with them and thus is phenetically similar to them. My second analysis of the suborder, which used hypothetical ancestors for several groups, placed Dromas with the charadriine waders (Fig. 35) on the basis of the lack of a posterior mandibular fossa; it is distinguished from them by a poorly-developed pneumatic fossa II of the humerus. Burton's (1974) report of the distribution of the Ap. $\mathrm{A}^{1}$ slip of $\mathrm{M}$. adductor externus supports the inclusion of Dromas in the Charadrii. (It is not known whether this slip occurs in the Lari.) The course of N. pterygoideus in Dromas, however, indicates that it is closer to the Lari than to the charadriine waders (Burton, pers. comm.). The leg scutellation of Dromas consists of large rectangular plates and is unlike that of any other wader of similar size, though it does resemble that of coursers and the larger gulls. Dromas also shares the presence of an ossified preethmoideum with the Glareolidae (omitting Pluvianus and Peltohyas) and some Lari. Its nesting behaviour and the appearance and behaviour of its 
chick seem most like those of the Lari. Dromas may prove closer to the latter than to any other group.

The inclusion of the Burhinidae in the Charadriiformes has long been debated, mostly because of their superficial resemblance to the Otididae and the uncertainties of the relationships of that family. On the basis of the morphology of the quadrato-tympanic articulation Lowe (1925) first placed the Burhinidae as a separate suborder in the Charadriiformes but later $(1931 b)$ decided that they are more gruiform. About half of his characters which purport to demonstrate the gruiform nature of the thick-knees also apply to other charadriiforms. He noted that the downy plumage pattern is similar to that of Haematopus but thought this only to "confirm the close relationships of the Gruae and Limicolae". Yudin (1965) thought their jaw musculature like the primitive Charadriinae and Cursorius and their skeletons half like bustards and half like plovers; he excluded them from the Charadriiformes. Kitto \& Wilson (1966) found the mobility of burhinid S-malate dehydrogenase like that of other charadriiforms. Jehl (1968) reaffirmed earlier observations that the downy plumage pattern is markedly different from that of bustards but like that of oystercatchers and other charadriiforms. Stepanyan (1970) reviewed the nonmorphological literature and thought it indicated a close relationship between the Burhinidae and the Charadriiformes. As noted above, Burton (1974, pers. comm.) found the course of N. pterygoideus in the Burhinidae to be the same as in Pluvianus, Pluvianellus, Chionis, the Haematopodidae, Charadriidae, and Recurvirostridae. Ahlquist (1974) found the IFPA patterns of Burhinus to resemble those of Haematopus, Recurvirostra, and some sandpipers more than those of bustards. He thought that the Burhinidae may be the most primitive group in the Charadriiformes.

Pluvianus has usually been placed in the Glareolidae; Seebohm (1886) lumped it with Cursorius. Lowe $(1931 b)$ thought the colour pattern of the chick almost exactly like that of adult Cursorius, which he took as "certain proof that Pluvianus is only a specialized courser". Burton (1974) found that its jaw musculature generally resembles that of the Glareolidae but that the course of N. pterygoideus is like that found in plovers. Yudin (1965) appears to be the first to recognize the distinctions between Pluvianus and the Glareolidae. He found it so different from the coursers and pratincoles, as well as from the "shorebirds proper", that additional investigations might show that it was not charadriiform. My analyses indicate that Pluvianus is not a glareolid and that the Burhinidae and Pluvianus are more closely related to each other than either is to any other group (Fig. 33). Their group is defined by a lacrimal-ectethmoid complex which abuts the jugal bar and holorhinal nostrils (Figs 5,7), character states which are probably functionally related. In agreement with my findings, Yudin (1965) thought that Pluvianus differs from other Charadriiformes "by precisely the same structural features as Burhinus". Other than the characters used in my analysis and the quite general trends noted by Yudin, I find little other evidence indicating a close relationship between Pluvianus and the Burhinidae; most of their similarities are those shared by other charadriine waders. On the other hand, Pluvianus does not seem particularly close to either the glareolids or the plovers. The relationship with the Burhinidae found here is probably best considered tentative until new evidence of its relationships is found.

Lowe (1931b) placed the Glareolidae, along with Dromas and Chionis, in his suborder Lari-Limicolae, his special category for groups which possess a combination of wader-like and gull-like features. He thought the forearm skeleton of Glareola gull-like and the skull 
a combination of gull- and plover-like character states. Moynihan (1956) thought that the hostile behaviour patterns of a captive individual of Glareola maldivarum resembled certain hostile displays of gulls and terns and that they might provide evidence of close relationship between the Glareolidae and Laridae. Bock (1964), impressed with the great similarity of the external and skull morphology of plovers and glareolids, thought that their placement in separate families should be reviewed. Yudin (1965) considered the Glareolidae an early branch of the line which gave rise to the Lari even though he found the wing muscles and tendons of Cursorius extremely similar to those of plovers. Jehl (1968) thought the downy plumage patterns of glareolids similar to those of plovers. Burton (pers. comm.) found that the course of N. pterygoideus in the glareolids is different from that in plovers. The IFPA patterns of the glareolids and plovers were "not readily separable" by Ahlquist (1974), who thought these groups closely related. My analyses show the Glareolidae to be charadriine waders (Fig. 33) but do not indicate to which group they are closest. They are defined by the contact or fusion of the ectethmoid and frontal and a raised, outward-twisted medial condyle of the quadrate. Pluvianus and Peltohyas were found not to be members of the Glareolidae.

Among the Charadrii only the coursers, pratincoles, Dromas, and the Lari have the path of N. pterygoideus between Parts M and O of M. pterygoideus (Burton, 1974, pers. comm.; Orenstein, pers. comm.), which appears to be the primitive condition for the order. Many specimens of pratincoles (including Stiltia) have a partially perforated proximal phalanx, digit III, a character state which defines the Lari. In the pratincoles the perforations appear in a thin delicate sheet of bone, are rough-edged, and have the appearance of damaged or not yet fully ossified areas; in the Lari the perforations are always sharp-edged, and there is no indication of ossification across them. Digit III is well-ossified in Dromas. The glareolids (but not Pluvianus or Peltohyas) share with Dromas and some of the Lari possession of an ossified preethmoideum. A similar-appearing structure is also found in some of the Jacanidae and Thinocoridae and may have been overlooked in other groups because it can easily be lost in the preparation of skeletons. The glareolids also share similarities of tarsal scutellation with Dromas and the Lari. Present evidence does not strongly indicate to which group the glareolids are most closely related.

Pluvianellus has been placed with the plovers ever since its discovery toward the end of the nineteenth century. Until Jehl's (1975) detailed study of its biology, no one who considered its relationships had seen the species in life. Boetticher (1934) thought it united characteristics of the plovers and lapwings but failed to describe them. Even casual examination of skins of the species is sufficient to show that it is unlike any known plover in bill and foot structure. Burton (1974) found the course of N. pterygoideus like that in plovers, a characteristic it shares with Chionis (Burton, pers. comm.). Jehl found that it differs from plovers in its body form, bill and leg morphology, foraging, territorial, courtship, and distraction behaviour, the relatively small size of its eggs, the semiprecocial nature of its chicks, its natal down, possession of a crop, and its dove-like drinking behaviour. He thought it like oystercatchers in its territorial and piping behaviour and in the feeding of its young, and like Chionis in general body form, bowing, threat, bill-wiping, and pre-copulatory behaviour, its small clutch size, the feeding of the young, and its possession of a crop. Jehl recommended that, until further studies are made, Pluvianellus be placed in a separate family. 
My analyses indicate that Pluvianellus and Chionis are members of a monophyletic line (Fig. 33) defined by lack of a flange on the prearticular process (Fig. 16) and possession of a knob on the extensor process of the metacarpus. The many similarities Jehl found between Pluvianellus and Chionis support this relationship.

Like their probable close relative Pluvianellus, the species of Chionis have proved enigmatic to students of the Charadriiformes. Shufeldt (1893) reviewed the literature on Chionis up to that time, in which various authors had argued that Chionis is closest to oystercatchers, glareolids, plovers, gulls, seedsnipes, and Dromas. Shufeldt's opinion on its relationships is itself a puzzle, since he advocated both that it is "one of the links among the plovers and gulls" and that its "nearest allies" are Haematopus and Glareola. Lowe $(1916 a, b, 1926)$ found Chionis to have a mixture of gull- and plover-like characteristics, placing it in his Lari-Limicolae (1931b) with Dromas and the Glareolidae. Boetticher (1934) thought Chionis closest to the Thinocoridae. On the basis of their similarities in jaw musculature, possession of a crop, and lack of toe webbing, Yudin (1965) thought Chionis and the seedsnipes close to each other, an opinion followed by Jehl (1968). Burton (1974) found that Chionis possesses an Ap. $\mathrm{A}^{1}$ slip of $\mathrm{M}$. adductor externus and that $\mathrm{N}$. pterygoideus passes through Part O of $\mathrm{M}$. pterygoideus (Burton, pers. comm.), states known only in the charadriine waders sensu lato. Ahlquist (1974) found the IFPA patterns and peptide maps of Chionis like those of the Lari, not especially like those of plovers, and little like those of seedsnipes. He thought that Chionis probably lies near the base of the Lari stem, perhaps near the skuas.

My characters indicate that the similarities of the Chionididae to the Thinocoridae and the Lari are restricted to the sharing of primitive states. The best evidence available indicates that they are closest to Pluvianellus.

The remaining line of the charadriine waders includes the species usually included in the Charadriidae (except Pluvianellus), Haematopodidae, Recurvirostridae, and Peltohyas (Fig. 33) and is defined by possession of a basipterygoid articulation of the pterygoid and basipterygoid processes on the basisphenoid rostrum (Fig. 10) and by fusion of the flange of the prearticular process of the mandible to the supraangular (Fig. 16). The close relationship among the plovers, lapwings, oystercatchers, Ibisbill, avocets, and stilts was recognised by Lowe (1931b), who placed them in his Charadriidae. Jehl (1968) thought them closely related on the basis of similarities of the downy plumage patterns. Burton (1974) found an entirely cartilaginous entoglossum only in the plovers, lapwings, oystercatchers, Ibisbill, avocets, and stilts. While Ahlquist (1974) found the IFPA patterns of Haematopus closest to those of plovers, he thought that his peptide maps indicated that the avocets and stilts are close to Numenius. The inadequacies of his data have been discussed elsewhere (Strauch, 1976). My evidence strongly indicates that the oystercatchers, Ibisbill, avocets, and stilts are modified plovers.

Early workers recognised Peltohyas as a plover, but Mathews (1913) placed it in the Glareolidae using characters which Bock (1964) found unconvincing. Indeed, Mathews \& Iredale (1921) admitted that the placement of Peltohyas in the Glareolidae might prove inaccurate. Unfortunately, Lowe $(1931 b)$, examining what must have been an imperfect or atypical skull, found that Peltohyas lacked basipterygoid processes and also placed it in the Glareolidae. Bock (1964) and Yudin (1965) have presented evidence that Peltohyas is a plover and not a glareolid. On the basis of a photograph and written description of a chick, Jehl (1968) argued that the colour pattern and feather structure of the downy 
plumage place Peltohyas in the Glareolidae. On the basis of sharing a condition of the insertion of M. pseudotemporalis superficialis otherwise unique to the plovers, as well as other features of the jaw musculature, Burton (1974) argued that Peltohyas is a plover. Maclean $(1973,1976)$ found that the behaviour of Peltohyas showed it to be a plover. The osteological evidence used in this study indicates that Peltohy'as is a plover.

The relationships among the charadriine waders are not yet well resolved. Five phyletic lines: the Burhinidae and Pluvianus; Dromas; the Glareolidae; Chionis and Pluvianellus; and the plovers and their allies, have been identified, but the relationships among them are unspecified by my character set. Dromas and the Glareolidae may prove closer to the Lari than to the charadriine waders, and the relationship between the Burhinidae and Pluvianus needs further examination. On the other hand, Chionis and Pluvianellus appear to be closely related, while the oystercatchers, Ibisbill, avocets, and stilts are found to be closely related to the plovers and lapwings.

The estimates of the phylogenetic relationships among the Charadriiformes presented here differ in many respects from traditional ideas. They are, however, based on consideration of more characters and species than any worker has used in the past. In addition, the patterns in the data have been analysed by a method which clearly displays their implications and which can be repeated with these and with new data. The relationships presented here can thus be tested by re-evaluation of the characters used here (by showing that the character state trees used are erroneous and, hence, that their phylogenetic implications are false) and by adding new data to the analyses and showing that some of the characters used here fail under a more severe test. Such work is already under way.

\section{ACKNOWLEDGEMENTS}

For their continuing advice, criticism, and encouragement I thank A. J. Baker, F. Cichocki, G. F. Estabrook, R. W. Storer, and W. H. Wagner, Jr. I am especially indebted to K. L. Fiala for his efforts in devising computer programs which made analysis of the large data set used in this study feasible.

P. J. K. Burton kindly supplied unpublished observations on the anatomy of several specimens unavailable to me. H. Abdulali (India), R. Summers (South Africa), J. G. Williams (Kenya), and the late $\mathrm{K}$. Thonglongya (Thailand) collected specimens especially for this study, and I am grateful to them.

The following kindly lent me many valuable specimens under their care: A. J. Baker and J. C. Barlow (Royal Ontario Museum), P. J. K. Burton (British Museum (Natural History)), P. Brodkorb (University of Florida), M. H. Clench and K. C. Parkes (Carnegie Museum), J. R. Jehl, Jr. (San Diego Society of Natural History (SDSNH)), N. K. Johnson (Museum of Vertebrate Zoology, University of California (MVZ)), A. C. Kemp (Transvaal Museum), F. C. Kinsky (New Zealand National Museum (NZNM)), W. E. Lanyon (American Museum of Natural History (AMNH)), A. R. McEvey (National Museum of Victoria), G. F. Mees (Rijksmuseum van Natuurlijke Historie (RVNH)), R. M. Mengel (University of Kansas Museum of Natural History), J. P. O'Neill (Louisiana State University Museum of Zoology), O. T. Owre (University of Miami (UM)), R. A. Paynter, Jr. (Museum of Comparative Zoology, Harvard University), S. M. Reed (Auckland Institute and Museum), C. G. Sibley (Peabody Museum of Natural History, Yale University), M. A. Traylor, Jr. (Field Museum of Natural History (FMNH)), and R. L. Zusi and S. L. Olson (U.S. National Museum (USNM)). This study was inspired and made possible by the large and valuable skeleton collection of The University of Michigan Museum of Zoology developed by R. W. Storer. 
I thank P. Hees and M. Orsen for their care in executing the skeletal illustrations. A. J. Baker, G. F. Estabrook, K. L. Fiala, and R. W. Storer read earlier drafts of the manuscript and made valuable comments on them. My wife, Elizabeth, prepared the non-skeletal illustrations, typed many versions of the manuscript, and ruthlessly edited all that I wrote.

I was supported during a portion of the study by a Rackham Block Fellowship. A Rackham Dissertation Grant provided funds for most of the skeletal drawings. The University of Michigan Computing Center provided computer facilities for this study.

\section{REFERENCES}

AHLQUIST, J. E. (1974). The relationships of the shorebirds (Charadriiformes). Ph.D. thesis, Yale University.

BAms, R. A. (1956). On the relation between the attachment of jaw muscles and the surface of the skull in Podiceps cristatus L., with some notes on the mechanical properties of this part of the head. II. Descriptive part. A. Proc. K. ned. Akad. Wet. (C) 59: 92-101.

BEDDARD, F. E. (1898). The structure and classification of birds. London: Longmans, Green.

BEDDARd, F. E. (1901). Notes upon the anatomy and systematic position of Rhynchaea. Proc. zool. Soc. Lond. 1901: $587-602$.

Bock, W. J. (1958). A generic review of the plovers (Charadriinae, Aves). Bull. Mus. comp. Zool. Harv. 118: 27-97. Bock, W. J. (1964). The systematic position of the Australian Dotterel, Peltohyas australis. Emu 63: 383-404.

Bock, W. J. \& McEver, A. (1969). Osteology of Pedionomus torquatus (Aves: Pedionomidae) and its allies. Proc. R. Soc. Vict. 82: 187-232.

BOETTICHER, H. VON. (1934). Kurze phylogenetisch-systematische Uebersicht der regenpfeiferartigen Vögel (Charadriiformes) und ihrer nächsten natürlichen Verwandten nach dem heutigen Stand unserer Kenntnisse. Kócsag 7: 1-25.

Boetticher, H. von. (1954). Note sur la classification des Vanneaux. Oiseall 24: 175-179.

BuRTON, P. J. K. (1971). Comparative anatomy of head and neck in the Spoon-billed sandpiper, Eurynorhynchus pygmeus and its allies. J. Zool., Lond. 163: 145-163.

Burton, P. J. K. (1972). Some anatomical notes on the Wrybill. Notornis 19: 26-32.

Burton, P. J. K. (1974). Feeding and the feeding apparatus in waders: a study of anatomy and adaptations in the Charadrii. London: Trustees of the British Museum (Natural History).

CAMIN, J. H. \& SoKal, R. R. (1965). A method for deducing branching sequences in phylogeny. Evolution 19: $311-326$.

Colless, D. H. (1967). The phylogenetic fallacy. Syst. Zool. 16: 289-295.

Colless, D. H. (1969a). The phylogenetic fallacy revisited. Syst. Zool. 18: 115-126.

Colless, D. H. (1969b). The interpretation of Hennig's "Phylogenetic Systematics"-a reply to Dr. Schlee. Syst. Zool. 18: 134-144.

Cracraft, J. (1968). The lacrimal-ectethmoid bone complex in birds: a single character analysis. Am. Midl. Nat. 80: 316-359.

CRACRAFT, J. (1972). The relationships of the higher taxa of birds: problems in phylogenetic reasoning. Condor 74: $379-392$.

CRACRAFT, J. (1974). Phylogeny and evolution of ratite birds. Ibis 116: 494-521.

ESTABROOK, G. F. (1968). A general solution in partial orders for the Camin-Sokal model in phylogeny. J. theor. Biol. $21: 421-438$.

EstabrooK, G. F. (1972). Cladistic methodology: a discussion of the theoretical basis for the induction of evolutionary history. Ann. Rev. Ecol. Syst. 3: 427-456.

Estabrook, G. F., Johnson, C. S., JR. \& McMorris, F. R. (1975). An idealized concept of the true cladistic character. Math. Biosci. 23: 263-272.

Estabrook, G. F., Johnson, C. S., JR. \& MCMorris, F. R. (1976a). A mathematical foundation for the analysis of cladistic character compatibility. Math. Biosci. 29: 181-187.

Estabrook, G. F., Johnson, C. S., JR. \& MCMorris, F. R. (1976b). An algebraic analysis of cladistic characters. Discrete Math. 16: 141-147.

Estabrook, G. F., Strauch, J. G., JR. \& Fiala, K. L. (1977). An application of compatibility analysis to the Blackiths' data on orthopteroid insects. Syst. Zool. 26: 269-276.

Fitch, W. M. \& Margoliash, E. (1967). Construction of phylogenetic trees. Science, Wash. 155: 279-284. 
Forbes, W. A. (1881). Notes on the anatomy and systematic position of the Jaçanas (Parridae). Proc. zool. Soc. Lond. 1881: 639-647.

Gadow, H. (1892). On the classification of birds. Proc. zool. Soc. Lond. 1892: 229-256.

GaDow, H. (1893). Vögel. II. Systematischer Theil. Bronn's Kl. Ordn. Tierreichs 6.

GARROD, A. H. (1873). On the value in classification of a peculiarity in the anterior margin of the nasal bones of certain birds. Proc. zool. Soc. Lond. 1873: 33-38.

Garrod, A. H. (1874). On some points in the anatomy of the Columbiae. Proc. zool. Soc. Lond. 1874: 249-259.

Garrod, A. H. (1877). Notes on the anatomy and systematic position of the genera Thinocortus and Attagis. Proc. zool. Soc. Lond. 1877: 413-418.

George, J. C. \& Berger, A. J. (1966). Avian myology. New York: Academic Press.

HANKe, B. \& Niethammer, G. (1955). Zur Morphologie und Histologie des Oesophagus von Thinocorus orbignyanus. Bonn. zool. Beitr. 6: 207-211.

Hennig, W. (1965). Phylogenetic systematics. A. Rev. Ent. 10: 97-116.

Hennig, W. (1966). Phylogenetic systematics. Urbana: Univ. Illinois Press.

Hoeven, J. VAN DER. (1868). Notice sur le Dromas ardeola Payk. Archs néerl. 3: 281-295.

Holman, J. A. (1961). Osteology of living and fossil New World quails (Aves, Galliformes). Bull. Fla St. Mus. 6: $131-233$.

Howard, H. (1929). The avifauna of Emeryville shellmound. Univ. Calif. Publs Zool. 32: 301-394.

Hudson, G. E. (1937). Studies on the muscles of the pelvic appendage in birds. Am. Midl. Nat. 18: 1-108.

Hudson, G. E., Hoff, K. M., Vanden Berge, J. \& Trivette, E. C. (1969). A numerical study of the wing and leg muscles of Lari and Alcae. Ibis 111: 459-524.

Hudson, G. E., Lanziliotn, P. J. \& Edwards, G. D. (1959). Muscles of the pelvic limb in galliform birds. Am. Midl. Nat. 61: 1-67.

HuxLex, T. H. (1867). On the classification of birds; and on the taxonomic value of the modifications of certain of the cranial bones observable in that class. Proc. zool. Soc. Lond. 1867: 415-472.

HuXLey, T. H. (1868). On the classification and distribution of the Alectromorphae and Heteromorphae. Proc. zool. Soc. Lond. 1868: 294-319.

JARDINE, N. (1969). The observational and theoretical components of homology: a study based on the morphology of the dermal skull-roofs of rhipidistan fishes. Biol. J. Linn. Soc. 1: 327-361.

JEHL, J. R., JR. (1968). Relationships in the Charadrii (shorebirds); a taxonomic study based on color patterns of the downy young. Mem. S. Diego Soc. nat. Hist. No. 3: 1-54.

JEHL, J. R., JR. (1975). Pluvianellus socialis: biology, ecology, and relationships of an enigmatic Patagonian shorebird. Trans. S. Diego Soc. nat. Hist. 18: 25-74.

JollIE, M. T. (1957). The head skeleton of the chicken and remarks on the anatomy of this region in other birds. J. Morph. 100: 389-436.

KitTo, G. B. \& Wirson, A. C. (1966). Evolution of malate dehydrogenase in birds. Science, N. Y. 153: 1408-1410.

Kluge, A. G. \& Farris, J. S. (1969). Quantitative phyletics and the evolution of the anurans. Syst. Zool. 18: 1-32.

Kozlova, E. V. (1961). [Trends of evolution in waders of the family Charadriidae on the basis of a study of the skull structure.] Trudy zool. Inst. Akad. nauk SSSR 29: 183-212. [In Russian.]

LeBEDINSKY, N. G. (1913). Beiträge zur Morphologie und Entwicklungsgeschichte des Vogelbeckens. Jena $Z$. Naturw. 50:647-774.

Le Quesne, W. J. (1969). A method of selection of characters in numerical taxonomy. Syst. Zool. 18: 201-205.

LOWE, P. R. (1915a). Coloration as a factor in family and generic differentiation. Ibis 1915: 320-346.

Lowe, P. R. (1915b). Studies on the Charadriiformes. I. On the systematic position of the Ruff (Machetes pugnax) and the Semipalmated sandpiper (Ereunetes pusillus), together with a review of some osteological characters which differentiate the Eroliinae (Dunlin group) from the Tringinae (Redshank group). Ibis 1915: 609-616.

Lowe, P. R. (1916a). Studies on the Charadriiformes. III. Notes in relation to the systematic position of the sheathbills (Chionididae). Ibis 1916: 122-155.

Lowe, P. R. (1916b). Studies on the Charadriiformes. IV. An additional note on the sheath-bills: some points in the oesteology of the skull of an embryo of Chionarchus "minor" from Kerguelen. V. Some notes on the Crab-plover (Dromas ardeola Paykull). Ibis 1916: 313-337.

Lowe, P. R. (1922). On the significance of certain characters in some Charadriine genera, with a provisional classification of the order Charadriiformes. Ibis 1922: 475-495. 
LOWE, P. R. (1923). Notes on the systematic position of Ortyxelus, together with some remarks on the relationships of the Turnicomorphs and the position of the Seed-snipe (Thinocoridae) and Sand-grouse. Ibis 1923: 276-299.

LOWE, P. R. (1925). (1) On the systematic position of the Jacanidae (jaçanás), with some notes on a hitherto unconsidered anatomical character of apparent taxonomic value. (2) A preliminary note on the classification of the Charadriiformes (Limicolae and Laro-Limicolae) based on this character, viz., the morphology of the quadrato-tympanic articulation. Ibis 1925: 132-147.

Lowe, P. R. (1926). More notes on the quadrate as a factor in avian classification. Ibis 1926: 152-188.

Lowe, P. R. (1931a). On the relations of the Gruimorphae to the Charadriimorphae and Rallimorphae, with special reference to the taxonomic position of Rostratulidae, Jacanidae, and Burhinidae (Oedicnemidae olim); with a suggested new order (Telmatomorphae). Ibis 1931: 491-534.

Lowe, P. R. (1931b). An anatomical review of the "waders" (Telmatomorphae), with special reference to the families, subfamilies, and genera within the suborders Limicolae, Grui-Limicolae and Lari-Limicolae. Ibis 1931: 712-771.

MCFarLane, R. W. (1963). The taxonomic significance of avian sperm. Int. orn. Congr. 13: 91-102.

Maclean, G. L. (1967). Die systematische Stellung der Flughühner (Pteroclidae). J. Orn., Lpz. 108: 203-217.

MaCifan, G. L. (1969). The sandgrouse-doves or plovers? J. Orn., Lpz. 110: 104-107.

Maclean, G. L. (1973). A review of the biology of the Australian desert waders, Stiltia and Peltohyas. Emu 73: $61-70$.

Maclean, G. L. (1976). A field study of the Australian dotterel. Emu 76: 207-215.

MCMorris, F. R. (1975). Compatibility criteria for cladistic and qualitative taxonomic characters. In Proceedings of the Eighth International Conference on numerical taxonomy. 399-415. Estabrook, G. F. (Ed.). San Francisco: W. H. Freeman and Co.

MAIllaRD, J. (1948). Recherches embryologiques sur Catharacta skua Brünn. Revue stitsse Zcol. 55: 1-144.

Marples, B. J. (1932). The structure and development of the nasal glands of birds. Proc. zool. Soc. Lond. 1932: $829-844$.

MARX, H. \& RABB, G. B. (1970). Character analysis: an empirical approach applied to advanced snakes. J. Zool., Lond. 161: 525-548.

Mathews, G. M. (1913). The birds of Australia 3. London: H. F. and G. Witherby.

Mathews, G. M. \& Iredale, T. (1921). Orders Casuarii to Columbae. A manual of the birds of Australia 1. London: H. F. and G. Witherby.

Moore, J. T. (1967). Elements of abstract algebra, 2nd ed. Toronto: Macmillan.

Moynihan, M. (1956). Behavior of a pratincole. Auk 73: 268-271.

Olson, S. L. (1970). A study of seedsnipe in southern South America by G. L. Maclean (A review). Bird-Banding $41: 258-259$.

Parker, W. K. (1890). On the morphology of the duck and auk tribes. Cunningham Mem. R. Irish Acad. No. 6: $1-132$.

PETERS, J. L. (1934). Check-list of birds of the world. 2. Cambridge, Massachusetts: Harvard University Press.

PRIM, R. C. (1957). Shortest connection networks and some generalizations. Bell Syst. Tech. J. 36: 1389-1401.

RAND, A. L. (1954). On the spurs on birds' wings. Wilson Bull. 66: 127-134.

Retzius, G. (1909). Die Spermien der Vogel. Biol. Unters. N.S. 14: 89-122.

Retzius, G. (1911). Zur Kenntnis der Spermien der Vogel. Biol. Unters. N.S. 16: 89-92.

SCHMDDT-NIELSEN, K. (1960). The salt secreting glands of marine birds. Circulation 21: 955-967.

SCHUMACHER, S. (1929). Zur Mechanik und Verwendungsart des Schnepfenschnabels. Z. Morph. Ökol. Tiere 15: 90-108.

SCLATER, P. L. (1880). Remarks on the present state of the Systema Avium. Ibis 1880: 340-350, 399-411.

Seebohm, H. (1886). A review of the species of the genus Cursorius. Ibis 1886: 115-121.

SеEвонм, H. (1895). Classification of birds; an attempt to diagnose the subclasses, orders, suborders, and families of existing birds. Supplement. London: R. H. Porter.

Selander, R. K. (1971). Systematics and speciation in birds. Avian biol. 1: 57-147.

SHARPE, R. B. (1891). A review of recent attempts to classify birds; an address delivered before the Second International Ornithological Congress on the 18th of May, 1891. Budapest.

SHARPE, R. B. (1896). Catalogue of the Limicolae in the collection of the British Museum. London: British Museum (Natural History).

Shoemaker, V. H. (1972). Osmoregulation and excretion in birds. Avian biol. 2: 527-574. 
Shufeldi, R. W. (1891). Contributions to the comparative osteology of Arctic and sub-Arctic waterbirds. Part 9. J. Anat. Physiol. 25: 509-525.

Shufeldt, R. W. (1893). The Chionididae. A review of the opinions on the systematic position of the family. Auk 10: 158-165.

Shufeldt, R. W. (1903). Osteology of the Limicolae. Ann. Carnegie Mus. 2: 15-70.

Sibley, C. G. \& AhlQuist, J. E. (1972). A comparative study of the egg white proteins of non-passerine birds. Bull. Peabody Mus. nat. Hist. No. 39: 1-276.

Sibley, C. G., Corbin, K. W. \& Ahlquist, J. E. (1968). The relationships of the Seed-snipe (Thinocoridae) as indicated by their egg white proteins and hemoglobins. Bonn. zool. Beitr. 19: 235-248.

Simpson, G. G. (1961). Principles of animal taxonomy. New York: Columbia University Press.

SNeAth, P. H. A. \& Sokal, R. R. (1972). Numerical taxonomy. San Francisco: W. H. Freeman and Co.

SPORNE, K. R. (1956). The phylogenetic classification of angiosperms. Biol. Rev. 31: 1-29.

StAaland, H. (1967). Anatomical and physiological adaptations of the nasal glands in Charadriformes birds. Comp. Biochem. Physiol. 23: 933-944.

SteBbins, G. L. (1974). Flowering plants: evolution above the species level. Cambridge, Massachusetts: Harvard University Press.

Stegmann, B. (1963). Der Processus internus indicis im Skelett des Vogelflügels. J. Orn., Lpz. 104: 413-423.

StegmanN, B. (1968). Uber die phyletischen Beziehungen zwischen Regenpfeifervögeln, Tauben und Flughühnern. J. Orn., Lpz. 109: 441-445.

Stegmann, B. (1969). Uber die systematische Stellung der Tauben und Flughühner. Zool. Jb. (Syst.) 96: 1-51.

StePANYAN, L. S. (1970). [Non-morphological criteria and their utilization for classification (Burhinidae (Aves) taken as an example).] Zh. obshch. biol. 31: 291-301. [In Russian, English summary.]

Storer, R. W. (1945). Structural modifications in the hind limb in the Alcidae. Ibis 87:433-456.

STORER, R. W. (1960). Evolution in diving birds. Int. orn. Congr. 12: 694-707.

StORER, R. W. (1963). Courtship and mating behavior and the phylogeny of the grebes. Int. orn. Congr. 13: 562-569.

Strauch, J. G., JR. (1976). The cladistic relationships of the Charadrifformes. Ph.D. thesis, The University of Michigan.

Stresemann, E. (1959). The status of avian systematics and its unsolved problems. Auk 76: 269-280.

Technau, G. (1936). Die Nasendrüse der Vögel. J. Om., Lpz. 84: 511-617.

VERHEYEN, R. (1961). A new classification for the non-passerine birds of the world. Bull. Inst. r. Sci. nat. Belg. 37 (27): $1-36$.

WAGNER, W. H., JR. (1961). Problems in the classification of ferns. In Recent advances in botany: 841-844. Toronto: University of Toronto Press.

WAGNER, W. H., JR. (1970). Biosystematics and evolutionary noise. Taxon 19: 146-151.

Walkinshaw, L. [H.] (1973). Cranes of the world. New York: Winchester Press.

Wolters, H. E. (1974). Aus der ornithologischen Sammlung des Museums Alexander Koenig. III. Bonn. zool. Beitr. 25: 283-291.

Yudin, K. A. (1965). [Phylogeny and classification of Charadriiformes.] Fauna SSSR (N.S.). No. 91. Birds. II. Part 1, No. 1. [In Russian, translated by Leon Kelso.]

Zusi, R. L. (1962). Structural adaptations of the head and neck in the Black Skimmer Rynchops nigra Linnaeus. Publ. Nuttall orn. Cl. No. 3: 1-8.

Zusi, R. L. \& Jehl, J. R., JR. (1970). The systematic relationships of Aechmorhynchus, Prosobonia, and Phegornis (Charadriiformes: Charadrii). Auk 87: 760-780. 